\title{
Understanding Trend Inflation Through the Lens of the Goods and Services Sectors
}

by Yunjong Eo, ${ }^{1}$ Luis Uzeda ${ }^{2}$ and Benjamin Wong ${ }^{3}$

${ }^{1}$ Department of Economics

Korea University, Seoul, South Korea 02841

${ }^{2}$ Canadian Economic Analysis Department Bank of Canada, Ottawa, Ontario, Canada K1A 0G9

${ }^{3}$ Department of Econometrics and Business Statistics Monash University, Caulfield East, Australia VIC 3145

yunjongeo@korea.ac.kr, Luzedagarcia@bankofcanada.ca, benjamin.wong@monash.edu

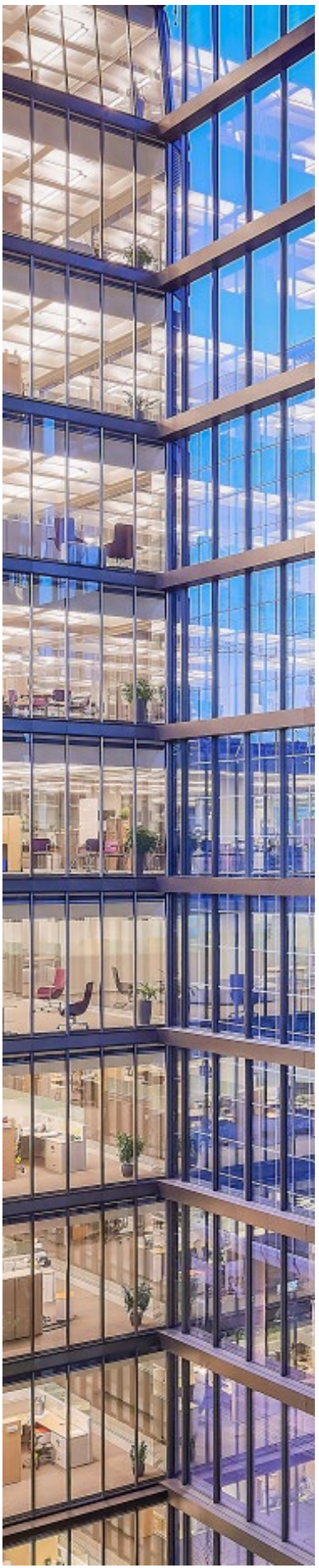

Bank of Canada staff working papers provide a forum for staff to publish work-in-progress research independently from the Bank's Governing Council. This research may support or challenge prevailing policy orthodoxy. Therefore, the views expressed in this paper are solely those of the authors and may differ from official Bank of Canada views. No responsibility for them should be attributed to the Bank. 


\section{Acknowledgements}

We thank Daniel de Munnik, José Dorich, Ippei Fujiwara, Matteo Luciani, James Morley, Rodrigo Sekkel, Saeed Zaman, Shang-Jin Wei, and Stefano Gnocchi, as well as conference and seminar participants at the Bank of Canada, the $26^{\text {th }}$ Symposium of the Society for Nonlinear Dynamics and Econometrics, the 2018 Bayesian Analysis and Modeling Summer Workshop at the University of Melbourne, the 2019 Workshop of the Australasia Macroeconomic Society, the 2020 Monash Macro/Finance Workshop and the Virtual Australian Macroeconomic Seminar for helpful comments and suggestions. This research is supported by the Australian Research Council grants (DP190100202 and DE200100693). The views expressed are those of the authors and do not necessarily reflect the position of the Bank of Canada. All remaining errors are our own. 


\section{Abstract}

We distinguish between the goods and services sectors in an otherwise standard unobserved components model of US inflation. Our main finding is that, while both sectors used to contribute to the overall variation in aggregate trend inflation, since the 1990s this variation has been driven almost entirely by the services sector. Two changes in sector-specific inflation dynamics are responsible for this finding: (i) a large fall in the variance of trend goods inflation; and (ii) the disappearance of comovement between trend goods and trend services inflation. Extensions to our baseline analysis by excluding energy prices and decomposing trend inflation into a common and a relative price component suggest a possible role of monetary policy in explaining our empirical findings. We also document similar changes in inflation dynamics internationally when extending our analysis to Australia and Canada.

Topics: Econometric and statistical methods; Transmission of monetary policy; Inflation and prices

JEL codes: C11, C32, E31, E52 


\section{Introduction}

A key aim of monetary policy is managing the persistent (or permanent) component of inflation (see, e.g., Mishkin (2007) and Draghi (2015)), a quantity often referred to as trend inflation. Indeed, a casual reading of any recent monetary policy report from the Federal Reserve Board makes it clear that, in addition to headline inflation, the Federal Reserve focuses on underlying (or core) measures of inflation that exclude, for instance, food and energy prices. This strategy is predicated on the belief that fluctuations in components such as food and energy are ultimately transitory and, consequently, should be excluded from monetary policy considerations about the trend (or long-run) path of inflation. A more recent development is that the two main components of inflation, namely the goods and services sectors, have been experiencing considerably different dynamics over the past three decades. Our goal in this paper is to understand how such contrasting behavior at the sectoral level manifests itself at the aggregate level of trend inflation dynamics.

To do so, we develop a two-sector unobserved components model with stochastic volatility (Two-Sector UC-SV, hereafter) that allows for time-varying comovements between trend inflation in the goods and services sectors in the US. A key antecedent paper for our work is that of Stock and Watson (2007), who proposed a univariate unobserved components model with stochastic volatility (UUC-SV, hereafter). Notably, the UUC-SV model represents the starting point for many contributions in the trend inflation literature. For instance, Chan, Koop and Potter (2013) extend the UUC-SV specification by proposing a version where trend inflation can exhibit bounded-drift dynamics. Stock and Watson (2016) extend their earlier work by leveraging a more granular dataset - the 17 components of personal consumption expenditure (PCE) inflation - to examine whether such a strategy improves in-sample and out-of-sample inferences of trend inflation. Mertens (2016) and Chan, Clark and Koop (2018) augment the UUC-SV setting by incorporating survey-based data, while Hwu and Kim (2019) assess the role of correlation between trend inflation and its transitory counterpart. At a high level, these studies all attempt to sharpen trend inflation estimates. In other words, their contributions are more closely related to econometric aspects of trend inflation measurement relative to the original UUC-SV framework. In this paper, we adopt 
Figure 1: Goods and Services Inflation Stylized Facts
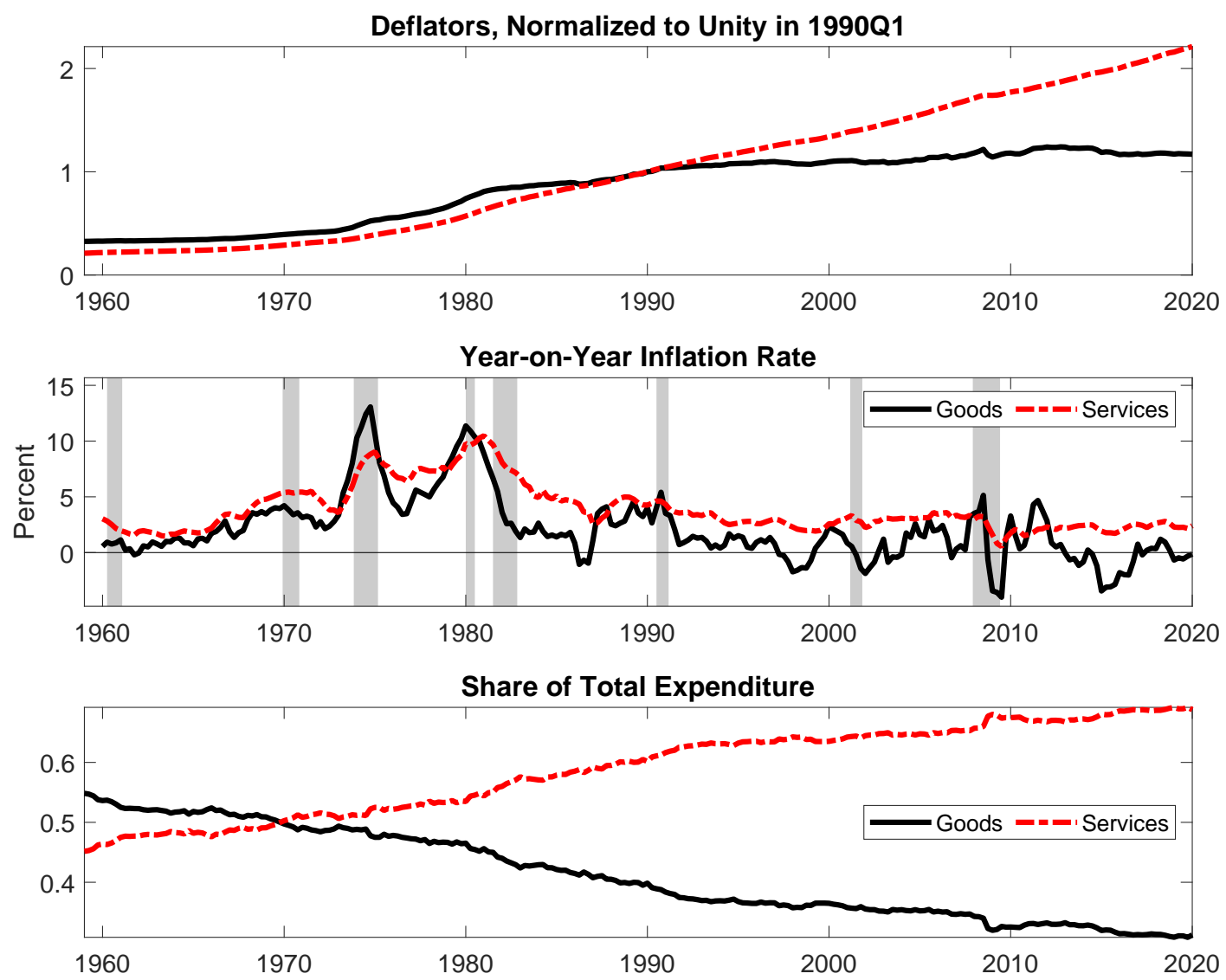

Notes: The shaded areas denote NBER recession dates. Expenditure shares sum to one.

a different, rather structural stance, to build on the work of Stock and Watson (2007). More specifically, while we preserve some key empirical features present in Stock and Watson (2007), our main concern is on the role of the goods and services sectors as sources of aggregate trend inflation dynamics.

To further motivate our decision to examine the implications of trend inflation through the lens of the goods and services sectors, Figure 1 presents stylized facts about the two sectors in the US economy. ${ }^{1}$ The top panel presents price deflators for both sectors, with the deflators normalized to unity in 1990Q1 for ease of comparison. The middle panel presents the year-on-year sector-specific inflation rates. ${ }^{2}$ The bottom panel presents the

\footnotetext{
${ }^{1}$ The data are taken from the categories of the US Personal Consumption Expenditure (PCE).

${ }^{2}$ We express these rates in year-on-year terms, as opposed to quarter-on-quarter, to better visualize broad
} 
nominal expenditure share on goods and services. We make three broad observations. First, while goods and services inflation moved roughly in sync till the mid 1980s, there are clear differences in their rates since the 1990s. From the deflators in the top panel, we can see that before the 1990s, prices in both sectors tended to grow at roughly the same rate. However, during the 1990s, a clear divergence emerges. While the price index of services has doubled since 1990Q1, the price index of goods has grown by only about one-fifth in the same period. ${ }^{3}$ Second, from the middle panel, goods inflation appears to be more volatile than services inflation. Whether goods inflation is more volatile than services is relatively unclear before the 1990s, it has almost certainly been true since then. Third, the share of consumption expenditure has switched. While they each had a roughly 50-50 split around 1970 , this share is now nearly $70 \%$ for services.

Our decision to model the trends in services and goods inflation goes beyond the differences in sector-specific inflation dynamics. In particular, a strand of the literature tries to understand the implications of inflation globalization for monetary policy as in Borio and Filardo (2007), Kamin, Marazzi and Schindler (2006), Bianchi and Civelli (2015) and Kamber and Wong (2020). In this sense, one could view goods and services inflation through the lens of traded and non-traded inflation. For instance, the flattening of the goods deflator in the last 30 or so years presented in Figure 1 coincides with the entry of China into the global consumer goods market, which is often viewed as the prima facie case for linking goods inflation to globalization in the last 30 years. Therefore, using the goods and services sectors to examine trend inflation dynamics should provide some perspective on the implications of globalization for the conduct of monetary policy.

Before we go further, we describe how we interpret trend inflation in our work. Stock and Watson (2007) decompose inflation into a permanent and a transitory "noise" component. The permanent component is then labeled "trend inflation". We adopt a similar transitory trends.

${ }^{3}$ The divergence between goods and services inflation has been noticed in previous works, such as Clark (2004) and Peach, Rich and Antoniades (2004). Nonetheless, these early commentaries may have been written with a perspective that goods inflation would recover, perhaps when the US dollar depreciated, and so implied minimal implications for thinking about trend inflation. Hindsight suggests that these divergences have instead further exacerbated since the early 2000s, motivating our effort to study their implications for aggregate trend inflation. 
noise component specification in our Two-Sector UC-SV model. ${ }^{4}$ Consequently, obtaining trend inflation in such a setting is conceptually closer to a removal-of-noise exercise, which is useful to obtain a signal about underlying and, ultimately, future inflation. There is also corroborating evidence that our interpretation of trend inflation is consistent with those within policy circles. ${ }^{5}$ However, we acknowledge an alternative strand of literature that views trend inflation as a time-varying inflation target (e.g., Kozicki and Tinsley (2001), Ireland (2007), Cogley, Primiceri and Sargent (2010), Coibion and Gorodnichenko (2011) and Ascari and Sbordone (2014)). While this body of work shares the label "trend inflation", the interpretation of trend inflation differs from ours. To be clear, we interpret trend inflation as a measure that provides a signal of future inflation through the removal of transitory noise. Hence, no equivalence is assumed a priori between our estimates of trend inflation and the monetary authority inflation target. We state our interpretation up front since this distinction does not seem to have been addressed explicitly elsewhere, and can be a source of confusion.

Our key result is the following: we find that variation in aggregate trend inflation is now entirely dominated by that in trend services inflation. This finding is a direct manifestation of several changes in inflation dynamics in both sectors, including (i) a fall in the correlation between trend goods and trend services inflation, which was moderate and positive in the 1970s, but is essentially zero today; and (ii) a change in the dynamics of goods inflation, where variation used to be partly permanent, but is now almost entirely dominated by transitory noise. We highlight two other findings in addition to our main result. First, after examining the post-Great Recession low inflation period, we find that our estimate of aggregate trend inflation has been consistently below the Federal Reserve's 2\% inflation target since 2012. This is the result of a fall in the level of trend goods inflation since 2014, and the level of trend services inflation being lower than pre-Great Recession levels. Second, our key result

\footnotetext{
${ }^{4}$ We experimented with modeling persistence in the transitory component, but these specifications were rejected by the data. Such issues are discussed in Section A2 of the Online Appendix.

${ }^{5}$ For example, in the minutes of the meeting of the Federal Open Market Committee held on June 1718, 2014, James Bullard from the St Louis Fed asks, "If inflation comes in at 1.9 percent and we've got underlying inflation at 1.75 percent, then should I say that we've got above-normal or above-trend inflation, or am I supposed to compare it with 2 percent, which is the Committee's official target?" To which Jeremy Rudd of the Federal Reserve Board replied, "In our judgment, you should be comparing it with 1.75 percent. We think $1 \frac{3}{4}$ percent is the underlying rate of inflation."
} 
is not specific to the US. When we extend our analysis to Australia and Canada, we find that aggregate trend inflation has also been dominated entirely by the services sector over the past 20 years.

The rest of the paper is structured as follows. Section 2 describes the Two-Sector UC-SV model. Section 3 presents the results from our model. Section 4 discusses policy implications of our results. Section 5 extends our analysis to Australia and Canada to explore whether our results are US-specific phenomena. Section 6 concludes.

\section{A Two-Sector Unobserved Components Model for Goods and Services Inflation}

We begin by describing our Two-Sector UC-SV model.

\section{$2.1 \quad$ Model}

In our model, goods inflation $\left(\pi_{t}^{G}\right)$ and services inflation $\left(\pi_{t}^{S}\right)$ are decomposed into their corresponding sector-specific permanent $\left(\tau_{t}^{G}, \tau_{t}^{S}\right)$ and transitory noise $\left(\zeta_{t}^{G}, \zeta_{t}^{S}\right)$ components. Our approach is akin to Stock and Watson (2007) and trend inflation work that builds directly on them, such as Mertens (2016) and Stock and Watson (2016). Formally, we have

$$
\begin{aligned}
& \pi_{t}^{G}=\tau_{t}^{G}+\zeta_{t}^{G}, \\
& \pi_{t}^{S}=\tau_{t}^{S}+\zeta_{t}^{S}
\end{aligned}
$$

We follow Stock and Watson (2007) and model trends as driftless random walk processes while abstracting from autoregressive dynamics for the noise processes, i.e.: ${ }^{6}$

\footnotetext{
${ }^{6}$ While modeling inflation's transitory components as noise may seem controversial, we make such an assumption for three reasons. First, for the sake of comparison, we wish to keep our approach as close as possible to Stock and Watson (2007), thereby retaining their interpretation that the estimation of trend inflation is essentially a noise-filtering exercise, as outlined in the Introduction. Second, we undertake a model comparison exercise and find that modeling sector-specific transitory components with an AR structure, or even as a time-varying AR process, does not outperform our baseline specification in a model comparison exercise. The results for this exercise are reported in the Online Appendix. Third, some of the results obtained when allowing for persistence appear very counter-intuitive. Consistent with our model comparison exercise, we conjecture that some of these counter-intuitive estimates for the transitory components are
} 


$$
\begin{gathered}
\tau_{t}^{G}=\tau_{t-1}^{G}+u_{t}^{\tau^{G}} \\
\tau_{t}^{S}=\tau_{t-1}^{S}+u_{t}^{\tau^{S}}
\end{gathered}
$$

Notably, the driftless random walk is a common modelling strategy in the trend inflation literature and appeals to the Beveridge and Nelson (1981) (BN) decomposition. The latter defines the trend component of time series as its long horizon forecast made at time $t$. Moreover, the $\mathrm{BN}$ decomposition also provides a natural link to trend inflation work that does not necessarily involve UC models, but rather constructs trend inflation appealing to the BN decomposition under different modeling frameworks (see, e.g., Cogley, Primiceri and Sargent (2010) and Kamber and Wong (2020)). Formally, for each sector $i$, we have

$$
\tau_{t}^{i}=\lim _{h \rightarrow \infty} \mathbb{E}_{t}\left[\pi_{t+h}^{i}\right]
$$

It is easy to verify that the driftless random walk assumption implies that the long horizon forecasts of $\pi_{t}^{G}$ and $\pi_{t}^{S}$ are given by $\tau_{t}^{G}$ and $\tau_{t}^{S}$, respectively.

Next, to allow for changes in the (conditional) volatility and correlation of the innovations to $u_{t}^{\tau^{i}}$ and $\zeta_{t}^{i}$ for $i=G, S$, we specify the following covariance structure:

$$
\left[\begin{array}{c}
\left(u_{t}^{\tau^{G}}, u_{t}^{\tau^{S}}\right)^{\prime} \\
\left(\zeta_{t}^{G}, \zeta_{t}^{S}\right)^{\prime}
\end{array}\right] \sim \mathcal{N}\left(\left[\begin{array}{c}
0_{2 \times 1} \\
0_{2 \times 1}
\end{array}\right] \quad\left[\begin{array}{cc}
\Omega_{\tau, t} & 0_{2 \times 2} \\
0_{2 \times 2} & \Omega_{\zeta, t}
\end{array}\right]\right)
$$

Note that a Cholesky factorization of $\Omega_{\tau, t}$ and $\Omega_{\zeta, t}$ yields

$$
\Omega_{j, t}=\left[\begin{array}{cc}
\sigma_{j^{G}, t}^{2} & \sigma_{j, t} \\
\sigma_{j, t} & \sigma_{j^{S}, t}^{2}
\end{array}\right]=\left[\begin{array}{cc}
1 & 0 \\
\gamma_{t}^{j} & 1
\end{array}\right]\left[\begin{array}{cc}
\exp \left(h_{t}^{j^{G}}\right) & 0 \\
0 & \exp \left(h_{t}^{j^{S}}\right)
\end{array}\right]\left[\begin{array}{cc}
1 & \gamma_{t}^{j} \\
0 & 1
\end{array}\right] \text { for } j \in\{\tau, \zeta\} .
$$

Therefore, in addition to sector-specific trends, we introduce six new state variables to capture the evolution of correlation $\left(\gamma_{t}^{\tau}\right.$ and $\left.\gamma_{t}^{\zeta}\right)$ and volatility $\left(h_{t}^{\tau^{G}}, h_{t}^{\tau^{S}}, h_{t}^{\zeta^{G}}\right.$, and $\left.h_{t}^{\zeta^{S}}\right)$ probably due to overfitting. 
associated with the behavior of goods and services inflation.

The block exogeneity assumption in (6) implies that the type of time-varying correlations we investigate in this paper are state specific. In other words, changes in sectoral correlation for the trend and transitory components are assumed to be independent phenomena. Of course, one could argue for letting the covariance matrix in Equation (6) be unrestricted, thereby allowing for cross-correlations between trend and transitory components. A drawback associated with such an approach is the well-known limitations to identifying covariance parameters within UC models - or state space models more broadly. In the interests of parsimony, we do not attempt to estimate correlations apart from the ones in $\Omega_{\tau, t}$ and $\Omega_{c, t}{ }^{7}$

For completeness, below we present the law of motion for the remaining state variables in our model:

$$
\begin{gathered}
h_{t}^{j^{i}}=h_{t-1}^{j^{i}}+u_{t}^{h^{j^{i}}}, j \in\{\tau, \zeta\}, i \in\{G, S\} \\
\gamma_{t}^{j}=\gamma_{t-1}^{j}+u_{t}^{\gamma^{j}}, j \in\{\tau, \zeta\} \\
{\left[\begin{array}{c}
\left(u_{t}^{h^{\tau^{G}}}, u_{t}^{h^{\tau^{G}}}, u_{t}^{h^{\zeta^{G}}}, u_{t}^{h^{\zeta^{S}}}\right)^{\prime} \\
\left(u_{t}^{\gamma^{\tau}}, u_{t}^{\gamma^{\zeta}}\right)^{\prime}
\end{array}\right] \sim \mathcal{N}\left(\left[\begin{array}{c}
0_{4 \times 1} \\
0_{2 \times 1}
\end{array}\right]\left[\begin{array}{cc}
\Omega_{h} & 0_{4 \times 2} \\
0_{2 \times 4} & \Omega_{\gamma}
\end{array}\right]\right),}
\end{gathered}
$$

where

$$
\Omega_{h}=\left[\begin{array}{cccc}
\sigma_{h^{\tau^{G}}}^{2} & 0 & 0 & 0 \\
0 & \sigma_{h^{\tau^{G}}}^{2} & 0 & 0 \\
0 & 0 & \sigma_{h^{c^{S}}}^{2} & 0 \\
0 & 0 & 0 & \sigma_{h^{c^{S}}}^{2}
\end{array}\right] \text {, and } \Omega_{\gamma}=\left[\begin{array}{cc}
\sigma_{\gamma^{\tau}}^{2} & 0 \\
0 & \sigma_{\gamma^{\zeta}}^{2}
\end{array}\right]
$$

Equations (1)-(9) describe a bivariate state space model, with measurement and state equa-

\footnotetext{
${ }^{7}$ Conceptually, our multivariate approach is related to Stock and Watson (2016), who use a factor structure with time-varying loadings to allow for interdependence in trend innovations across a 17-sector model. For ease of interpretation, instead of using time-varying loadings on a common factor, we model the evolution of the covariance matrix directly. From the perspective of the number of parameters one needs to estimate, for specification up to four sectors, our parametrization is more parsimonious. In a 17-sector specification, where numerosity of the parameters in an unrestricted covariance matrix would make estimation of time-varying correlations prohibitive, their parametrization via a factor approach is more parsimonious and computationally tractable.
} 
tions given by Equations (1)-(2) and (3)-(9), respectively.

Similarly to Stock and Watson (2016), we calculate aggregate trend inflation, denoted $\tau_{t}$, by aggregating the sector-specific trend inflation,

$$
\tau_{t}=\omega_{G, t} \tau_{t}^{G}+\omega_{S, t} \tau_{t}^{S}
$$

where $\omega_{G, t}$ and $\omega_{S, t}$ are the expenditure weights of goods and services, respectively, and $\omega_{t}^{G}+\omega_{t}^{S}=1$. Note that these weights are not estimated; we obtain them from the nominal expenditure shares reported in Figure 1.

\subsection{Data and Estimation}

Our Two-Sector UC-SV model constitutes a nonlinear state space model, and so, as is common in this literature, we conduct estimation using Bayesian methods. The states are estimated using precision sampling methods described in Chan and Jeliazkov (2009). In

particular, the (log) volatility states - i.e. $h_{t}^{\tau^{G}}, h_{t}^{\tau^{S}}, h_{t}^{\zeta^{G}}$, and $h_{t}^{\zeta^{S}}-$ are estimated combining precision sampling with the auxiliary mixture sampler of Omori et al. (2007). The priors are standard relative to the extant trend inflation literature. A detailed description on the estimation and the priors is given in Section A1 of the Online Appendix. Our data for goods and services inflation are taken from the seasonally adjusted deflators of the US personal consumption expenditure (PCE), with the weights based on nominal expenditure shares. We obtain all the data through the FRED dataset. ${ }^{8}$ To convert the respective goods, services, or PCE deflators to inflation rates for our model, we annualized the difference of the logarithm of the price deflators, which is an approximately annualized quarter-on-quarter percent change in the deflator. Our sample is from 1959Q1 to 2020Q1. 
Figure 2: Estimated Level and Volatility of Trend Inflation
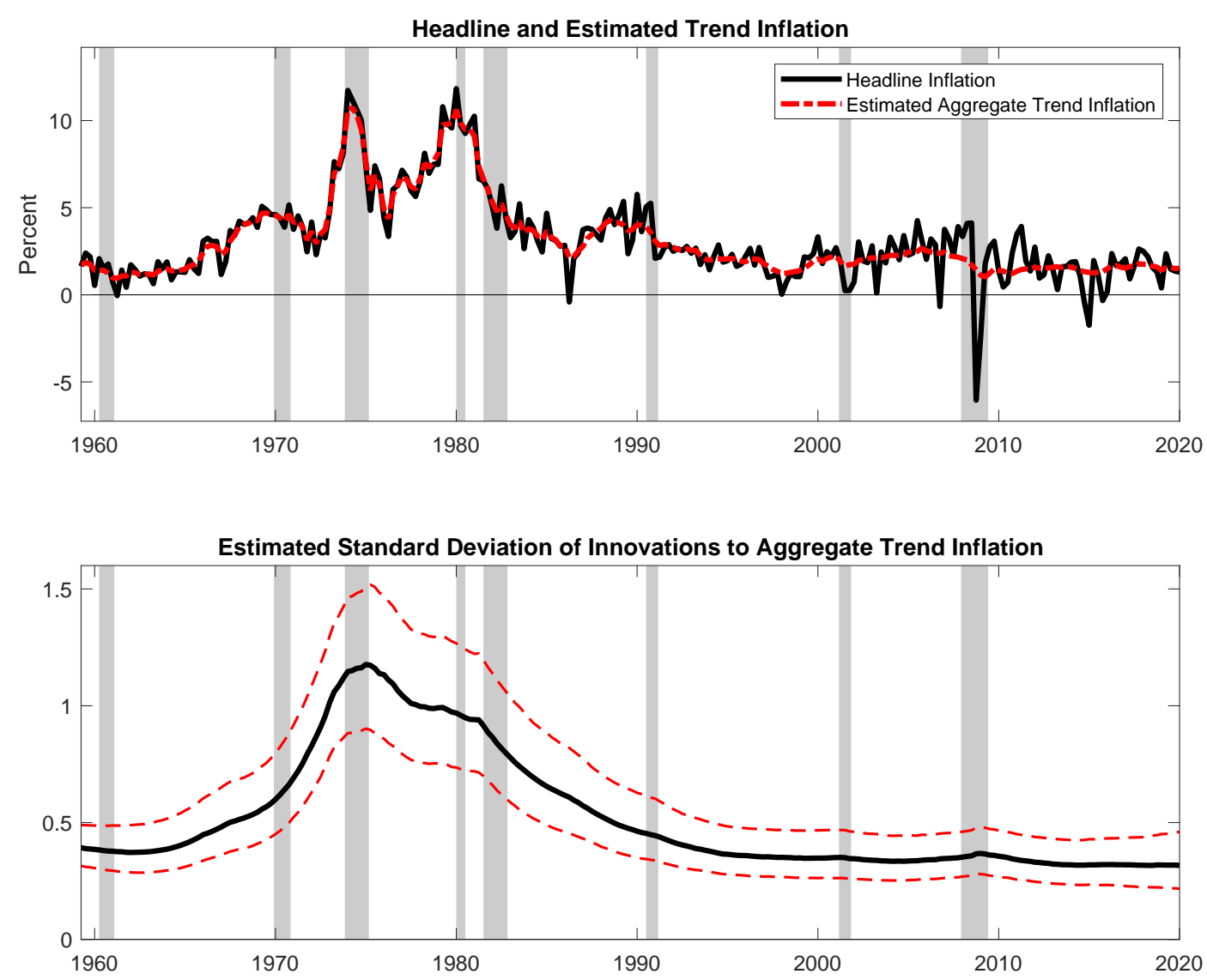

Notes: The shaded areas denote NBER recession dates. All units are in terms of annualized quarter-onquarter inflation. Top Panel: Headline inflation is plotted against the posterior estimate of aggregate trend inflation. Bottom panels: Estimated standard deviation of innovations to aggregate trend inflation. Posterior median estimate with associated $67 \%$ credible set.

\section{Results from the Two-Sector UC-SV Model}

In what follows, we begin to present implications from our model to aggregate trend inflation. The top panel of Figure 2 plots the (smoothed) estimates of aggregate trend inflation from the Two-Sector UC-SV model together with PCE inflation. We report posterior medians,

\footnotetext{
${ }^{8}$ The FRED mnemonics for the goods and services deflators are DGDSRD3Q086SBEA and DSERRD3Q086SBEA, respectively. If we require the PCE deflator, for example when we estimate the UUC-SV model, we use DPCERD3Q086SBEA. The FRED mnemonics for nominal expenditures for goods and services are DGDSRC1 and PCESV, respectively. We construct the weights as the proportion of the nominal expenditure for goods or services divided by the sum of nominal expenditure for goods and services.
} 
relegating the associated credible sets to the Online Appendix. ${ }^{9}$ We point out, however, that estimation of trend inflation is sufficiently precise, in the sense that posterior credible intervals are not excessively wide. ${ }^{10}$ The inflation rates and estimated trend inflation are both in terms of annualized quarter-on-quarter inflation, consistent with the data that enter the model.

Overall, our trend inflation estimate broadly mimics the history of US post-war inflation. In particular, trend inflation peaks during the Great Inflation in the 1970s and begins to disinflate in the early to mid-1980s. Since then, trend inflation has been persistently low. We also observe that, while episodes of large swings in quarter-on-quarter headline inflation occurred after the 1990s, trend inflation remained low and stable. The bottom panel of Figure 2 presents our estimate for trend inflation (time-varying) volatility. Specifically, we present the estimated (conditional) standard deviation of the innovations to aggregate trend inflation with their associated $67 \%$ posterior credible set. ${ }^{11}$ Such a result shows that there has been a large fall in the variance of the permanent component of aggregate inflation since the late 1970s. In particular, trend inflation volatility exhibits a hump shape that captures its rise and subsequent fall during the 1970-1980 window. All in all, our results for both the level and the volatility of US trend inflation are very much in line with what is documented in Stock and Watson (2007) based on the UUC-SV model. ${ }^{12}$ In Section A3 of the Online Appendix, we also document out-of-sample comparisons relative to the UUC-SV. In short, our approach produces very similar - and in some instances even slightly superior - forecasting performance relative to the UUC-SV model.

We now move on to present the key results that we obtain from our Two-Sector UCSV model. The discussion starts with the sector-specific levels of trend inflation, with a particular focus on what our model implies for aggregate and sector-specific trends during

\footnotetext{
${ }^{9}$ Point estimates throughout this paper denote posterior medias unless when explicitly stated otherwise.

${ }^{10}$ Our decision to omit selected credible sets are simply to facilitate the exposition of our results when reporting such sets would clutter figures with too many estimates. Again, the reader is referred to the Online Appendix for a complete inspection of uncertainty surrounding our estimates.

${ }^{11}$ Expanding Equation (11) and applying the variance operator, we can calculate the standard deviation of the innovations to aggregate trend inflation, where $\operatorname{std}\left(\Delta \tau_{t}\right) \approx$ $\sqrt{\omega_{G, t}^{2} \operatorname{Var}\left(u_{t}^{\tau^{G}}\right)+\omega_{S, t}^{2} \operatorname{Var}\left(u_{t}^{\tau^{S}}\right)+2 \omega_{G, t} \omega_{S, t} \gamma_{t}^{\tau} \operatorname{std}\left(u_{t}^{\tau^{S}}\right) \operatorname{std}\left(u_{t}^{\tau^{G}}\right)}$.

${ }^{12}$ Using the UUC-SV model, Eo (2016) also finds the hump-shaped pattern on the variance of innovations to trend inflation for CPI inflation and GDP deflator inflation.
} 
Figure 3: Sectoral Trend and Headline Inflation
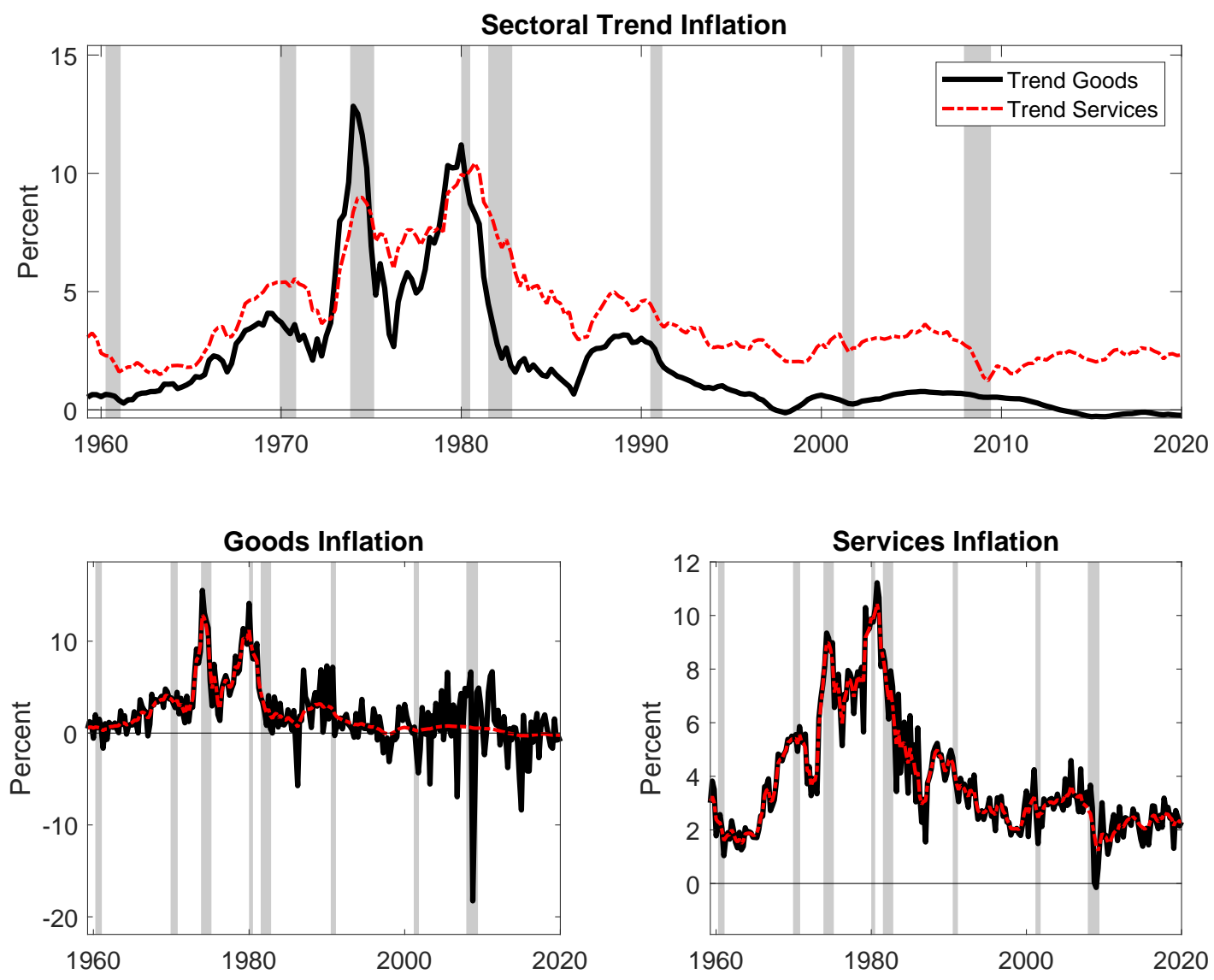

Notes: The shaded areas denote NBER recession dates. Trend inflation estimates are obtained as the posterior median estimates from our model. Units in terms of annualized quarter-on-quarter inflation.

the aftermath of the Great Recession of 2007-09. We then turn to presenting the sources of aggregate trend inflation volatility, where we show how variation in aggregate trend inflation has become entirely dominated by variation in trend services inflation.

\subsection{Sector-Specific Inflation Dynamics from the Two-Sector Model}

Figure 3 presents our trend estimates for sector-specific and aggregate inflation. ${ }^{13}$ The top panel presents the estimated trends in both sectors, while each bottom panel reproduces these

\footnotetext{
${ }^{13}$ Again, to conserve space, we report the associated credible sets of the respective trend inflation estimates in the Online Appendix. We do note, however, that the credible sets associated with our estimate of aggregate and sector-specific trend inflation are fairly precisely estimated.
} 
estimates alongside inflation in each sector. All results are once again in terms of annualized quarter-on-quarter inflation. Notably, the dynamics of sector-specific and aggregate trend inflation are quite different when we contrast the 1970s to early 1980s period with the period from the mid-1980s onwards. In particular, during the 1970s, both trend services and trend goods inflation are roughly equivalent to actual goods and services inflation, respectively. In contrast, the latter part of our sample witnesses marked differences in the dynamics of sector-specific trend inflation. More precisely, since the 1990s or so, while goods inflation has been fluctuating at a relatively higher frequency, its sector-specific trend has moved very little. The dynamics of trend services inflation, on the other hand, continued to track services inflation quite closely. The sharp changes in the dynamics of goods inflation will manifest themselves in various guises as we present more results later on.

Returning to the top panel of the level of trend inflation in each sector, over the full sample, trend services inflation has often been above trend goods inflation, apart from the two periods in the 1970s where inflation accelerated greatly. These two periods coincide with the first and second oil shocks in 1973-1974 and 1979-1980, respectively. Given that much of energy consumption is classified as goods, the period of the two oil price shocks probably saw these large oil price increases feed directly into trend goods inflation. Indeed, as observed in Figure 3, trend goods inflation tracked goods inflation almost one for one during the 1970s. The two oil price shocks probably underlie why trend goods inflation briefly exceeded the level of trend services inflation in the 1970s. Since the 1980s, however, trend services inflation has been consistently above trend goods inflation. The gap between the level of trend services and trend goods inflation has been quite stable since the 1990s, although we note that since the Great Recession of 2007-09, it appears that the gap between sectoral trends may be widening. While calculating the optimal inflation rate is out of the scope of our paper, we note in passing that if such a gap remains or continues to widen, this may have implications for finding the optimal level of inflation (or the inflation target). For example, in general equilibrium settings such as Wolman (2011), the dispersion of inflation across both sectors matters for welfare-related calculations. 
Figure 4: Aggregate and Sectoral Trend Inflation since the Great Recession
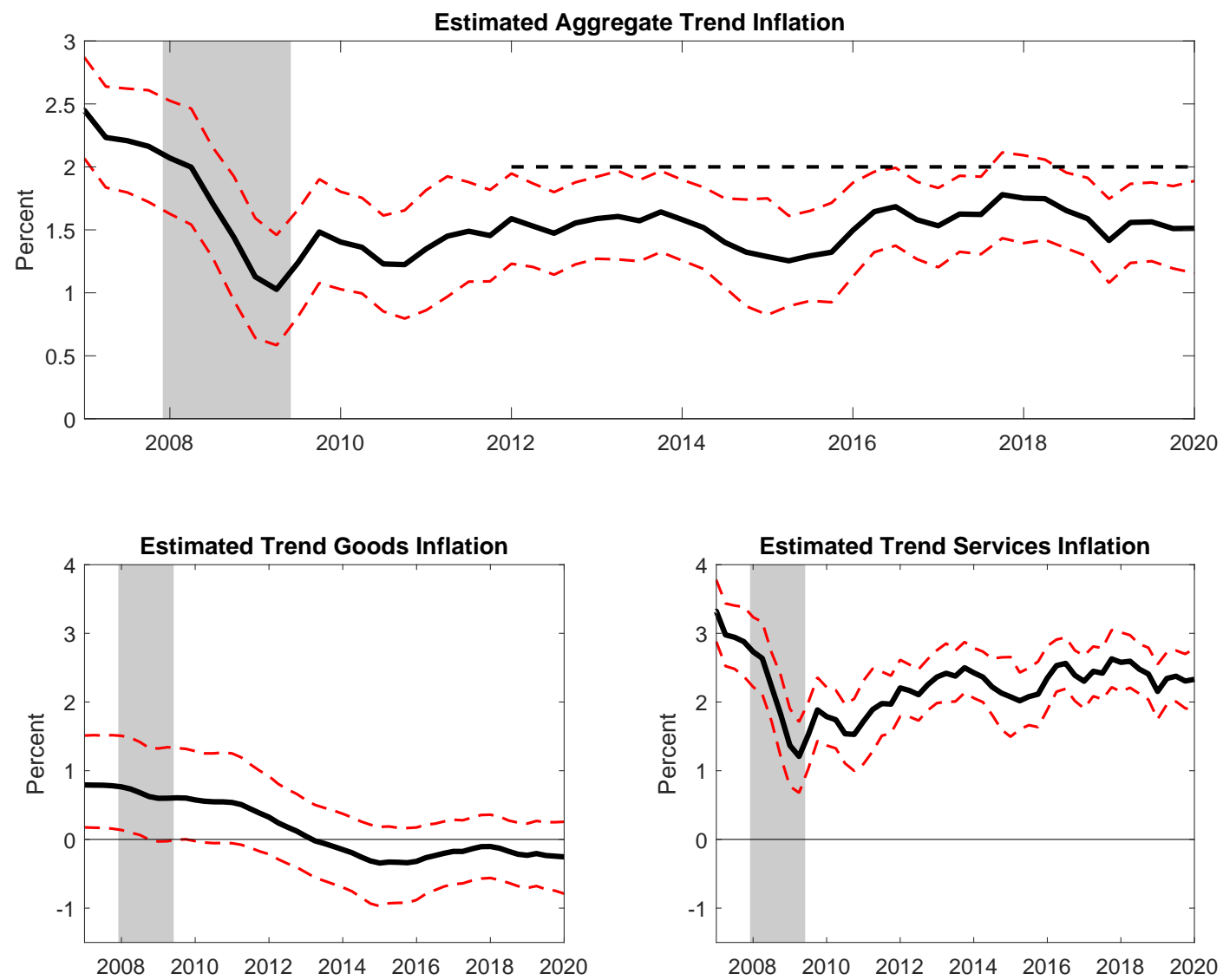

Notes: The shaded areas denote NBER recession dates. Trend inflation estimates are obtained as the posterior median estimates from our model. Units in terms of annualized quarter-on-quarter inflation. Dotted lines represent $67 \%$ credible sets.

\section{Low Inflation Since the Great Recession}

We now focus on the period since 2007 to shed light on the low inflation outcomes since the Great Recession. Inflation has been notably low - even compared to that before the Great Moderation - over the past decade, with the debate centered on whether this low-inflation episode is more of a permanent or transitory phenomenon (see, e.g., Andolfatto and Spewak (2019)). The period of low inflation has sparked deflationary concerns, especially if some of these falls were permanent. One reason for concern is the perceived role of monetary policy to stabilize trend inflation, perhaps anchored to an explicit numerical inflation target. Since 2012, the Federal Reserve has adopted an explicit numerical inflation target of $2 \%$. To 
explore such issues, the top and bottom panels of Figure 4 present, respectively, our previous estimates of aggregate and sector-specific trend inflation - with their associated $67 \%$ credible intervals - from 2007Q4 until 2020Q1. We also mark out in our panel for aggregate trend inflation the $2 \%$ inflation target.

Our posterior median estimate of aggregate trend inflation has always been under $2 \%$ since the Great Recession, and it has remained so even after the introduction of an explicit inflation target by the Federal Reserve in 2012. The upper bound of our credible interval for aggregate trend inflation has almost always been under or at $2 \%$ from 2009 onwards, often with more than $80 \%$ of the posterior density estimate being under $2 \%$ since 2009 . While we note that $2 \%$ is sometimes marginally within the credible set towards the end of the sample, we urge caution with any such interpretation for two reasons. First, the posterior median is still below $2 \%$, and so our point estimate of aggregate trend inflation still sits under the inflation target. ${ }^{14}$ Second, the revelation of future data may lead to two-sided (or smoothing) algorithms - such as the one adopted in this paper - to potentially revise these trends inflation estimates. In particular, revisions from smoothing algorithms tend to be more drastic the closer the observations are to the end of the sample.

Turning to the sector-specific trend inflation, the fall in aggregate trend inflation, at least in the immediate aftermath of the Great Recession, is to a large extent due to a fall in trend services inflation. We note that while trend services inflation has been gradually rising since 2011, this level is low relative to that before the Great Recession (see Figure 3). On the other hand, trend goods inflation appeared stable during and through the immediate aftermath of the Great Recession, but experienced a large fall around 2013, and has stayed either negative or very close to $0 \%$ since. Such a fall has partially offset the modestly rising trend services inflation. It is worth noting that, while the timing of a fall in trend goods inflation may have been in part driven by the collapse in crude oil prices from late 2014 to 2016, fluctuations in crude oil prices do not mechanically translate into changes for trend goods inflation. For example, crude oil prices also collapsed during the Great Recession and bounced back thereafter, although trend goods inflation stayed roughly flat through the Great Recession and its immediate aftermath.

\footnotetext{
${ }^{14}$ Our results are broadly unchanged if reporting posterior means instead of medians.
} 
Figure 5: Estimated Conditional Standard Deviation and Correlation of Innovations
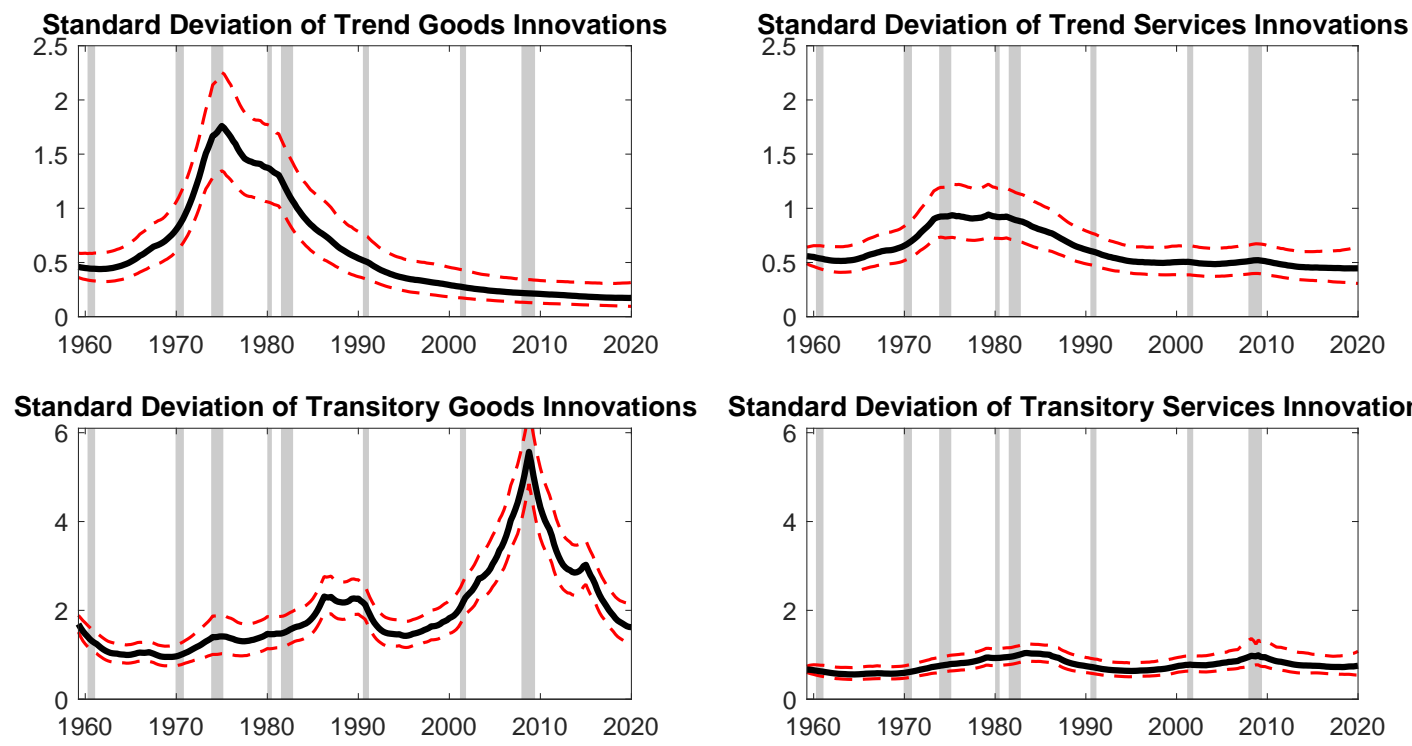

Standard Deviation of Transitory Services Innovations
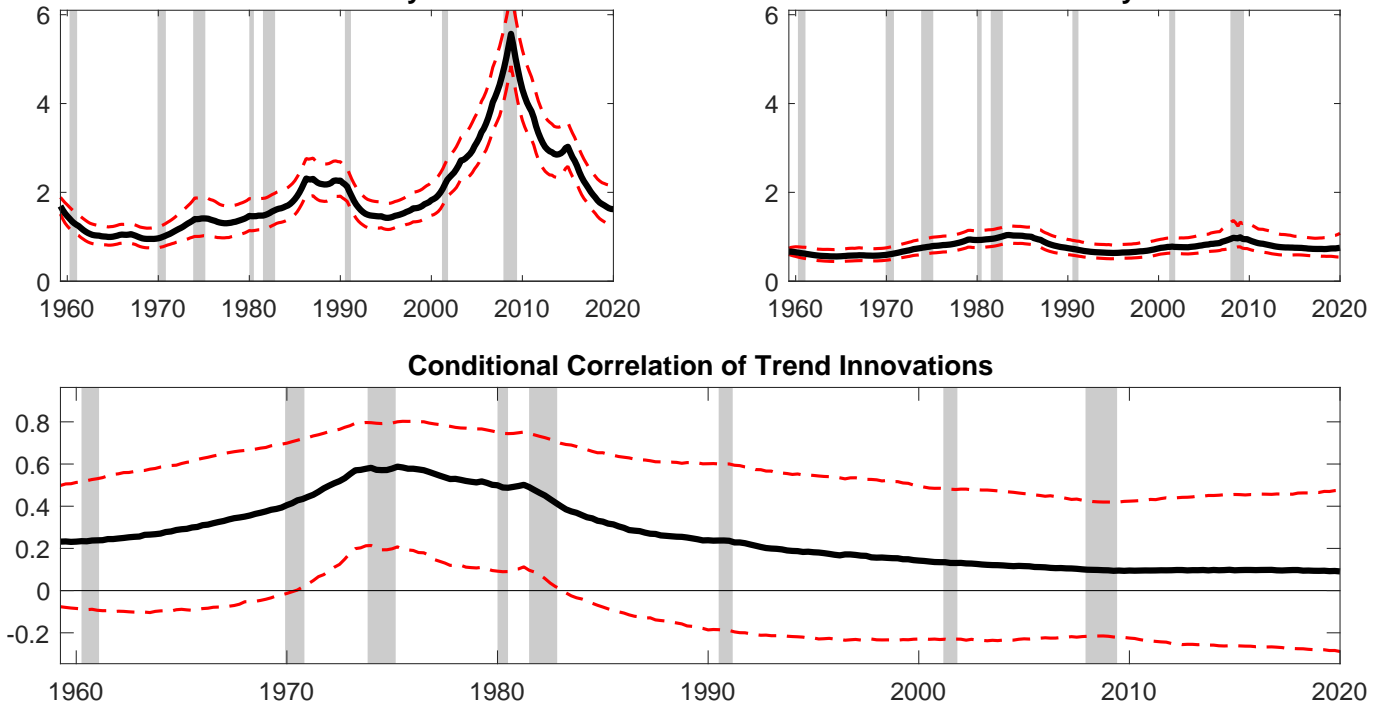

Notes: The shaded areas denote NBER recession dates. All estimates denote posterior medians with their associated $67 \%$ posterior credible interval.

All in all, viewing the low aggregate trend inflation outcomes since the Great Recession through the sector-specific lens suggests richer sector-specific dynamics at play. In particular, it appears both sectors do contribute to aggregate trend inflation below $2 \%$.

\subsection{Sources of Variation in Aggregate Trend Inflation}

We now turn to presenting results on the sources of variation in aggregate trend inflation. 


\section{Changing Volatility of Sector-Specific Trend Inflation}

We focus first on the estimated volatility of inflation in both sectors. The top two panels of Figure 5 present the estimated time-varying standard deviation of the innovations to trend goods and services inflation (i.e. $\operatorname{std}\left(u_{t}^{\tau^{G}}\right)$ and $\left.\operatorname{std}\left(u_{t}^{\tau^{S}}\right)\right)$. Akin to aggregate volatility, both sectors also display a hump-shaped pattern. That is, the volatility of sector-specific trend inflation starts as low to moderate at the start of the sample, rises in the period of the Great Inflation in the 1970s and falls around the early 1980s, remaining low ever since.

While it is true that both the volatility of trend services and trend goods inflation display a hump-shaped pattern, such a pattern is much sharper and more pronounced in the goods sector, so much so that our posterior estimates for $\operatorname{std}\left(u_{t}^{\tau^{G}}\right)$ are about twice that of $\operatorname{std}\left(u_{t}^{\tau^{S}}\right)$ at the height of the Great Inflation. Towards the end of the sample, the standard deviation of trend goods inflation is close to zero, and only about one-fifth of the standard deviation of trend services inflation. Notably, the standard deviation of innovations of trend goods inflation being close to zero more recently is consistent with the near-constant dynamics for trend goods inflation over the past 20 years, as documented earlier in Figure 3.

For completeness, we present the volatility of the transitory noise component in both sectors. The middle two panels of Figure 5 presents the estimated time-varying standard deviation of the sector-specific transitory noise components. Overall, we see little discernible pattern with the volatility of the transitory component of services inflation. In contrast, the volatility of the transitory component of goods inflation appears to be changing over time. In fact, our results suggest that transitory goods inflation has become increasingly more volatile over the years, peaking at the Great Recession, subsequently returning to the values observed in the Great Moderation toward the end of the sample. This is an important point to note, because the volatility of overall goods inflation has historically been a significant order of magnitude larger than the overall volatility of services inflation. ${ }^{15}$

Nonetheless, we note that, while we document volatility changes associated with permanent and transitory components of goods inflation, the overall volatility of goods inflation has remained fairly stable over time. In other words, the sum of the conditional standard

\footnotetext{
${ }^{15}$ In Section A5 of the Online Appendix, we show that much of this transitory noise component reflects energy prices.
} 
deviation of the permanent and transitory components of goods inflation is more or less time invariant. ${ }^{16}$ Our findings thus suggest that what has changed is the composition of overall variation in goods inflation in terms of what is mapped to permanent and transitory movements. In particular, permanent changes represent a smaller proportion of the overall variation in goods inflation more recently.

\section{Changing Correlation of Trend Innovations}

The bottom panel of Figure 5 presents the estimated time-varying correlation between the trend innovations. ${ }^{17}$ Our estimates indicate that correlation was modest before the 1970s, but steadily rose during the Great Inflation. Thereafter, with the 1980s disinflation, the correlation in both sectors fell steadily, and indeed, continued to fall even after the 1990s. We find this correlation is essentially close to zero in the last decade or so, which means that trend services and trend goods inflation are essentially uncorrelated. We note, from the bounds of our credible interval, that the correlation between the trend innovations does not appear to be very precisely estimated, in the sense that our credible interval often encompasses zero. Even so, our model supports, with a high degree of probability, that this correlation is not only moderate to high, but probably was not zero during the period of the Great Inflation in the 1970s.

The fact that the Great Inflation was the only period where we find a significant correlation between the innovations to trend goods and trend services is indicative that the effects of common shocks might be less salient nowadays relative to, say, sector-specific shocks. Such a result has been documented in studies by, e.g., Foerster, Sarte and Watson (2011) and Garin, Pries and Sims (2018). In terms of common shocks, monetary policy stands out as a plausible candidate and is consistent with Cecchetti et al. (2007)'s account that the conduct of monetary policy is the source of the large fall in the variance of the permanent component during the early 1980s documented in Stock and Watson (2007). Energy price

\footnotetext{
${ }^{16}$ Given the innovations to trend and the transitory component are orthogonal, the variance of the innovations to overall sector-specific inflation is just the sum of the variances of both components.

${ }^{17}$ It is easy to show from Equation 7 that time-varying correlation estimates, $\rho_{t}^{j}$, can be computed by

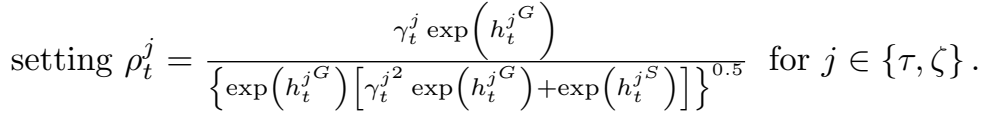


shocks are another plausible candidate for these common shocks, given the timing of the Great Inflation. In Section 4.1, we investigate further the sources of these common shocks, but for now, just to preempt some further results, we can rule out energy price shocks as being the source of common shocks behind comovements between services and goods trends.

\subsubsection{Decomposing the Variation of Aggregate Trend Inflation}

We now explore the key sources of time variation which we uncover from the Two-Sector UC-SV model in explaining variation in aggregate trend inflation. Equations (3) and (4) imply

$$
\Delta \tau_{t}^{i}=u_{t}^{\tau^{i}}, \quad i=\{G, S\}
$$

By expanding on Equation (11), the variance of aggregate trend inflation from the TwoSector UC-SV model can be written as

$$
\operatorname{Var}\left(\Delta \tau_{t}\right) \approx \omega_{G, t}^{2} \operatorname{var}\left(u_{t}^{\tau^{G}}\right)+\omega_{S, t}^{2} \operatorname{var}\left(u_{t}^{\tau^{S}}\right)+2 \omega_{G, t} \omega_{S, t} \operatorname{Cov}\left(u_{t}^{\tau^{G}}, u_{t}^{\tau^{S}}\right)
$$

where the approximation accounts for the possibility that the weights (i.e. $\omega_{G, t}$ and $\omega_{S, t}$ ) may change from $t-1$ to $t$. However, any approximation error is likely to be trivial given that we know from the bottom panel of Figure 1 that quarter-on-quarter change in weights, if any, is very small. Equation (13) thus shows that the variance of the innovation to aggregate trend inflation can be approximated by decomposing it into a linear sum of three components consisting of the variance of the innovations to the goods and services sector, and the covariance term to account for the correlation between the two sectors. Recall that the two key sources of time variation in the Two-Sector UC-SV are (i) the variance of the innovation to the trend component of goods and services; and (ii) the correlation between trend innovations to both sectors. These sources of time variation which we uncover from our Two-Sector UC-SV will, therefore, by construction, have implications for the composition of the overall variance of aggregate trend inflation, since all such elements appear in Equation (11).

Figure 6 presents our decomposition of the estimated variance of aggregate trend inflation into the three linear components implied by Equation (13), which we denote Goods, Services, 
Figure 6: Decomposition of Volatility of Aggregate Trend Inflation

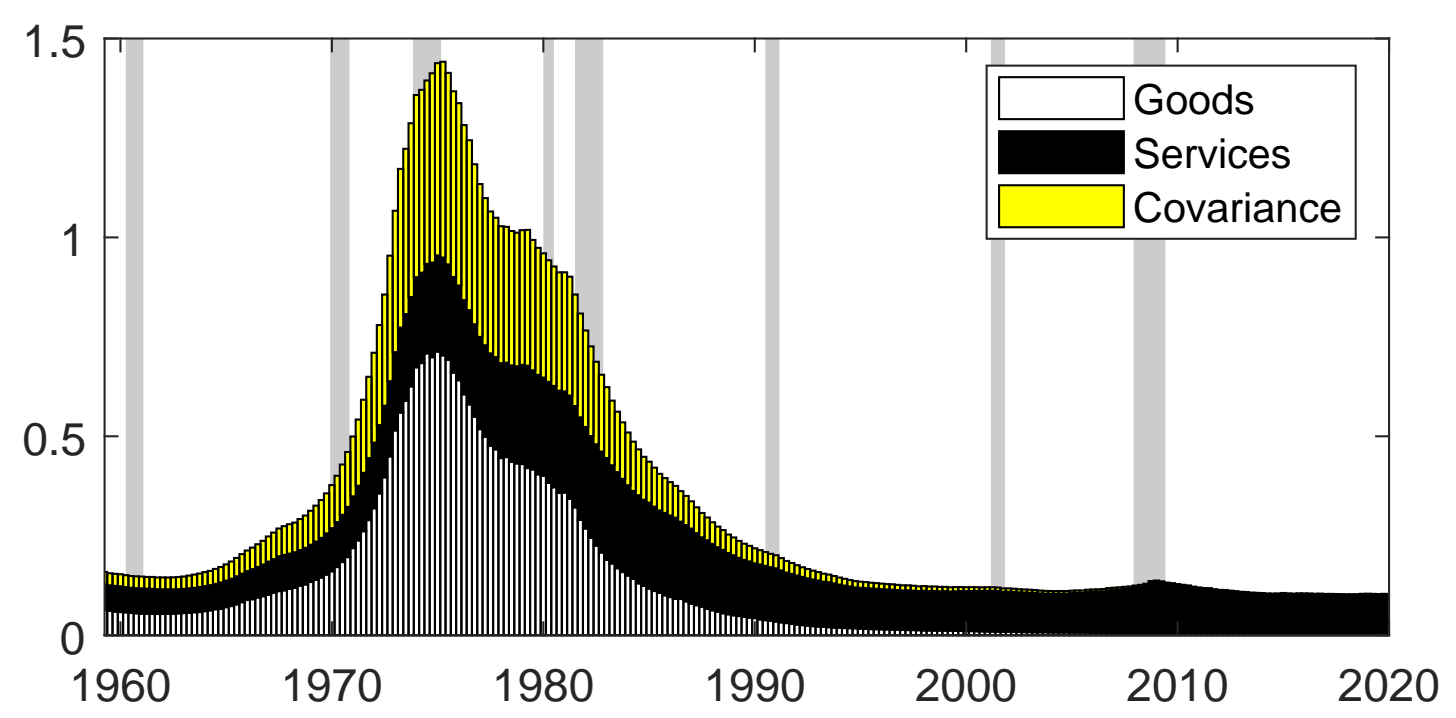

Notes: The shaded areas denote NBER recession dates. Trend inflation is in units of annualized quarter-onquarter inflation. Goods, services, and covariance refer to the decomposition of the components of aggregate trend inflation as presented in Equation (13).

and Covariance. From the height of the stack bars, we once again observe a hump shape, where the hump represents a high variance for aggregate trend inflation during the Great Inflation and the subsequent disinflation, as we documented in the bottom panels of Figure 2. Also, it is clear that all three components have always contributed to the total variance of aggregate trend inflation, and especially so during the Great Inflation. Nevertheless, since the 1990s, the goods and covariance components no longer contribute to variation in aggregate trend inflation. The contribution of the variance of services sector today is slightly larger relative to the Great Inflation, in part reflecting the increase in the expenditure weights offsetting some of the fall in the variance in the innovations to trend services. However, in sum, the large drop in the contribution of trend goods and the covariance terms has meant that since the 1990s, not only has absolute aggregate trend inflation volatility fallen, but this now reduced variance has become almost entirely dominated by the variance of trend services inflation. 


\section{Discussion and Interpretation of Results}

To recap, our key result is that variation in aggregate trend inflation has, since around the 1990s, seen a composition shift from being partly driven by both trend goods and services inflation to being fully dominated by variation in trend services inflation. Moreover, we showed two key changes in sector-specific inflation dynamics that can help reconcile our result: (i) a large fall in the variance of trend goods inflation to the point there is almost no variation in trend goods inflation; and (ii) a decrease in the correlation between innovations to trend goods and services inflation to the point that there is now no correlation.

In this section, we first extend our analysis to understand whether common factors, such as energy price shocks or the conduct of monetary policy, can explain changes in the inflation dynamics. Investigating the role of possible common shocks is a natural step when one considers that changes in sector-specific dynamics are in part due to a decline in comovements between trends in both sectors. Subsequently, we discuss some policy implications that arise from our results, reconciling them with some of the broader literature.

\subsection{Was there a Role for Energy Prices or Monetary Policy?}

As mentioned throughout this paper, we find that a fall in the correlation of innovations to trend goods and services partly drives our key result. Given that the timing of such a fall coincided with the 1970s oil shocks, one could naturally posit that energy shocks may be influencing our results. To investigate the role of energy, we reconstructed indices for both the goods and services sectors excluding energy, or ex-energy for short. The idea is that if energy is a key driver of our findings, we should see a large deviation from our baseline results once we reconduct the estimation using ex-energy variants of goods and services inflation. Energy comprises about $6 \%$ in the PCE basket, with almost all of it (over 90\%) classified as energy goods, with a much smaller role for energy services. We construct sectoral inflation rates excluding energy components in each sector through chain weighting. The components we exclude in constructing these ex-energy indices are "Gasoline and other energy goods" in the goods sector and "Gas and electric utilities" in the services sector. ${ }^{18}$

\footnotetext{
${ }^{18}$ These are categories in the National Income and Product Accounts (NIPA) data, which we use to construct these ex-energy indices. We relegate details on how we reconstruct these ex-energy indices as well
} 
Figure 7: Results from Excluding Energy
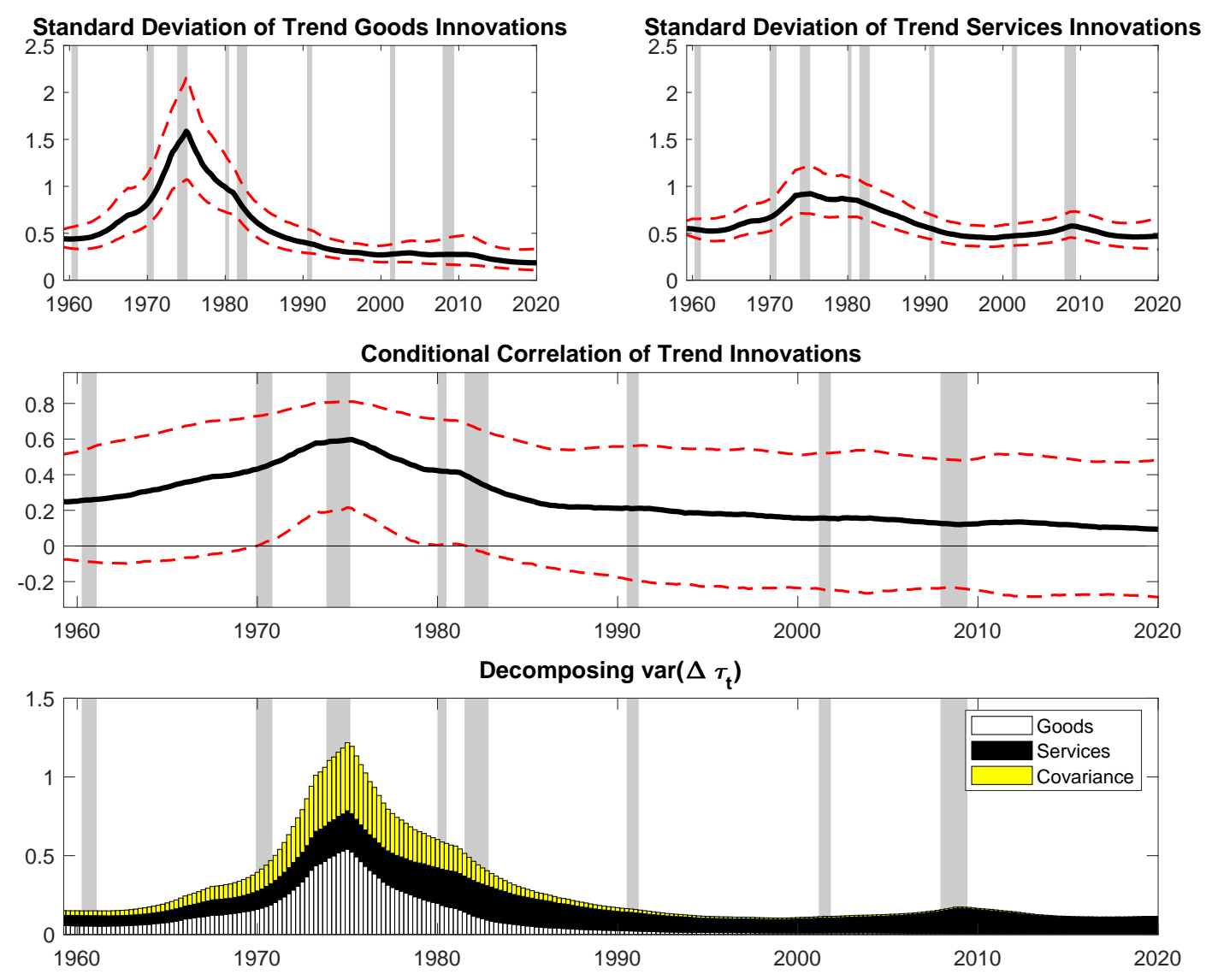

Notes: The shaded areas denote NBER recession dates. All estimates denote posterior medians with their associated $67 \%$ posterior credible interval. Goods, services, and covariance refer to the decomposition of the components of aggregate trend inflation as presented in Equation (13).

Figure 7 presents various results from our model, where we re-estimate our Two-Sector UC-SV model with goods excluding energy and services excluding energy. The top two panels present estimates of the time-varying standard deviation for trend goods excluding energy and trend services excluding energy. The middle panel presents the time-varying correlation of the innovations to trend goods and services when we exclude energy. Qualitatively, these results are very similar to those that we obtain in Figure 5. We note, however, that quantitatively the estimate of the volatility of trend goods inflation is slightly smaller at the peak when we exclude energy. This is somewhat unsurprising, as energy consumption is as less important results when we exclude energy to Section A5 of the Online Appendix. 
mostly related with the goods sector. In addition, results for the decomposition of aggregate trend inflation, when excluding energy prices, are also qualitatively very similar to what we obtain in Figure 6. We therefore conclude that our key insights are robust to the exclusion of energy goods and energy services.

At the same time, robustness to excluding energy components suggests that changes in trend inflation dynamics may be related to different common drivers. At a minimum, correlation reflects the presence of common drivers. In particular, Cecchetti et al. (2007) interprets the fall in overall trend inflation volatility reported in Stock and Watson (2007) as reflecting the conduct of monetary policy. To evaluate this hypothesis, we go a step further and conduct an exercise to identify the 'pure' component of inflation. More precisely, we adopt the idea of decomposing inflation into a pure inflation and a relative price change component as proposed by Reis and Watson (2010) and apply it to our estimates of sectoral trends. Pure trend inflation is the common component in inflation changes that has an equiproportional effect on all inflation rates of goods and services in the long run. The idea behind pure inflation is that if the monetary authority increases the money supply by $\mathrm{x} \%$, then all prices should go up by $x \%$ in the long run. This intuition is closely related to the quantitative theory of money, and thus provides us with a mechanism to identify the role for the conduct of monetary policy and link it to changes in our estimates for aggregate trend inflation. In developing the decomposition framework, we follow Reis and Watson (2010) and Wolman (2011) and cast each sector-specific trend as the sum of two components as follows:

$$
\begin{aligned}
\tau_{t}^{G} & =f_{t}+\tau_{t}^{G *}, \\
\tau_{t}^{S} & =f_{t}+\tau_{t}^{S *},
\end{aligned}
$$

where $f_{t}$ denotes pure trend inflation that is common to both sectors, and $\tau_{t}^{G *}$ and $\tau_{t}^{S *}$ capture the relative trend inflation for goods and services, respectively. These three latent 
Figure 8: Decomposition into Pure Inflation and Relative Price Components
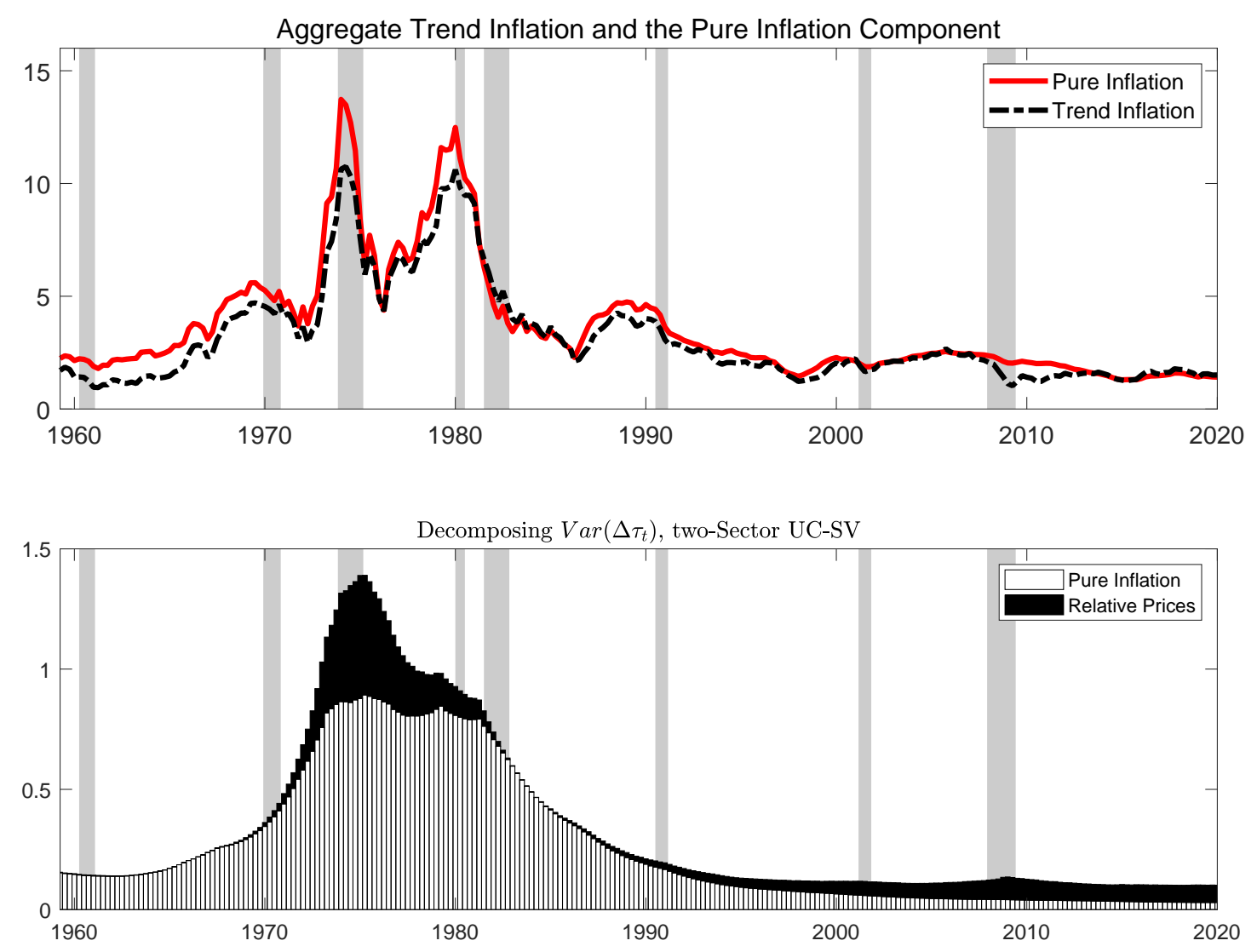

Notes: The shaded areas denote NBER recession dates. Trend inflation is in units of annualized quarteron-quarter inflation.

components evolve as follows:

$$
\begin{aligned}
f_{t} & =f_{t-1}+\nu_{t}^{f}, \\
\tau_{t}^{G *} & =\tau_{t-1}^{G *}+\nu_{t}^{\tau^{G *}}, \\
\tau_{t}^{S *} & =\tau_{t-1}^{S *}+\nu_{t}^{\tau^{S *}} .
\end{aligned}
$$

In the interest of brevity, we leave details on how we implement the identification of the pure inflation component to Section A4 of the Online Appendix. But we briefly state that because Equations (14) to (18) imply a state space representation, by treating our estimates for sector-specific trends as observables, we can extract the pure inflation component applying 
a Kalman smoother. ${ }^{19}$ We perform the signal extraction exercise based on the posterior medians of the sectoral trend inflation rates and their associated variances and covariance over time.

Figure 8 presents results based on the state space representation in Equations (14) to (18). The top panel of Figure 8 shows that the level of pure inflation appears to closely align with aggregate trend inflation over time. This indicates that the pure inflation component was largely responsible for the two high inflation episodes in the $1970 \mathrm{~s}^{20}$ We therefore interpret this as evidence that the conduct of monetary policy was - to a considerable extent - responsible for the Great Inflation episode in the 1970s. This interpretation is also in line with other studies, such as Orphanides (2004) and Boivin and Giannoni (2006).

The bottom panel of Figure 8 reports results from a variance decomposition akin to Equation 13 applied to our representation of sectoral trends in terms of a pure inflation and a relative price component. It shows that a large fall in the variance of trend inflation in the late 1970s and early 1980s was ultimately associated with the decrease in the variance of the pure inflation component. This suggests, in line with Cecchetti et al. (2007), that monetary policy played a prominent role in the disinflation episode that took place in the beginning of the 1980s.

\subsection{Policy Implications}

We discuss two policy implications that derive from our findings: first, the inferences of underlying inflation in a policy environment, and second, the reconciling of our results by lending some perspective to the globalization of inflation debate.

\footnotetext{
${ }^{19}$ We adopt a Kalman smoother here, rather than its (one-sided) filter version, to remain consistent with our estimation approach discussed in Section 2.2. In other words, we estimate our Two-Sector UC-SV model using Bayesian methods which provide, by construction, smoothed (or two-sided) estimates for all state variables.

${ }^{20}$ Reis and Watson (2010) find, using their dynamic factor model, the increase in headline inflation in the early 1970s and its declines in the 1990s were mostly attributed to their estimated changes in the relative price factor. However, they do not allow for stochastic volatility in the model, so the standard deviation of changes in the pure inflation component is estimated to be 0.3 percent, whereas our estimated standard deviations once allowing for stochastic volatility range from 0.2 percent since 2000 to 0.9 percent in the 1970s and 1980s. Thus, their constant variance specification might underestimate variations of pure inflation components and its role in the increase of inflation in the 1970s and 1980s. See the bottom panel of Figure 8 for the variance decomposition in our model.
} 
Our key result, that aggregate trend inflation is now entirely dominated by trend services inflation, does imply one straightforward implication within policy environments such as central banks: namely, gaining a read on underlying inflation. To be precise, we define underlying inflation as just inflation excluding transient noise, which is consistent with the interpretation of trend inflation in our model. Such a quantity is naturally related to forecasts of inflation, given that transient noise is expected to dissipate, and therefore if current inflation is above (below) underlying inflation, one should expect inflation to fall (rise) in the future. This is one reason why the Federal Reserve tracks PCE excluding food and energy inflation. They typically regard food and energy inflation to be ultimately transitory and therefore it should not feed into underlying inflation. The perspective of underlying inflation as filtering inflation of transitory noise is not entirely new and has been taken as the objective of part, though we note not all, of the core inflation literature (see, e.g., Bryan and Cecchetti (1994) and Cogley (2002)). In this paper, we use the term underlying inflation rather than core inflation, given the latter term has a wide variety of definitions and interpretations within the broader literature.

We find that while goods inflation remains volatile, variation in goods inflation no longer contributes to variation in aggregate trend inflation. This means that a policymaker observing a movement in goods inflation in real time should conclude that such a fluctuation likely represents transient noise with regards to obtaining a read on whether underlying inflation has changed. On the other hand, a fluctuation in services inflation is more likely to represent a signal of a change in underlying inflation. We note that our results are also consistent with work from the Federal Reserve. For example, Tallman and Zaman (2017), in a forecasting exercise, shows a link between economic slack and services, but not goods, inflation. Luciani (2020) also shows that inflation dynamics in the goods sector tend to reflect sector-specific idiosyncratic dynamics. In this regard, our results are consistent with the fact that it is variation in the services sector which provides a signal of a change in the overall inflation environment and that variation in goods inflation is perhaps too noisy for such purposes.

Finally, we briefly touch on how our results may provide some perspective on the role of globalization of inflation hypothesis. At the heart of the hypothesis is that inflation is increasingly globally determined, an idea typically attributed to Borio and Filardo (2007). 
As we discussed in the Introduction, the goods and services dichotomy can be viewed as an approximation to the tradeable and non-tradeable split in consumer expenditures. If one is willing to accept the goods and services as the traded and non-traded inflation split, with goods representing the component of inflation which is driven by international factors, our results would suggest that very little of this variation feeds directly into variation in trend inflation. To the extent that trend inflation is a quantity which central banks seek to stabilize, our results suggest that much of this variation in trend inflation is still very much domestically driven by the services sector.

\section{$5 \quad$ International Evidence}

We apply our model to international data to explore whether the results we obtain are more generalizable. To this end, we estimated our model using Australian and Canadian data. ${ }^{21}$ For Australia, our sample covers 1976Q1-2020Q1, and for Canada, 1961Q2-2020Q1. ${ }^{22}$ We stress that we did not set out to just explore Australia and Canada. In fact, such a sample choice is purely driven by limitations of the data coverage for other comparable economies. We point out, though, that Australia and Canada do possess some interesting features which at least mark them out as useful points of comparison relative to our benchmark results for the US. First, both Australia and Canada had similar inflation experiences to the US, in the sense that the Great Inflation saw very high inflation rates in both countries, and these inflation rates fell and became very stable in the 1990s. In particular, both the Reserve Bank of Australia and Bank of Canada adopted an explicit inflation target in 1997 and 1992, respectively, whereas the inflation target for the US was always implicit until it was made explicit in 2012. Therefore, a comparison with the US can provide some perspective on whether our key results are US-specific or extend to different inflation targeting regimes.

\footnotetext{
${ }^{21} \mathrm{~A}$ major challenge for seeking international evidence is that few economies retain long time series of goods and services inflation which are either constructed using consistent methodology or have been rendered consistent by their respective statistical agencies to the level of the US data. For example, for the UK, we are unable to go back beyond 1981, and for New Zealand, 1987. It is important to have long time series because the thrust of our key results is to make comparisons relative to the period of the Great Inflation in the 1970s. At a minimum, we require the sample coverage of the international data to at least include the rise and then fall of inflation to at least be compared to the key results which we obtain for the US.

${ }^{22}$ We leave details on how we construct the goods and services deflator, as well as some more auxiliary results, for both economies to the Online Appendix.
} 
Figure 9: Aggregate and Sectoral Trend Inflation for Australia and Canada
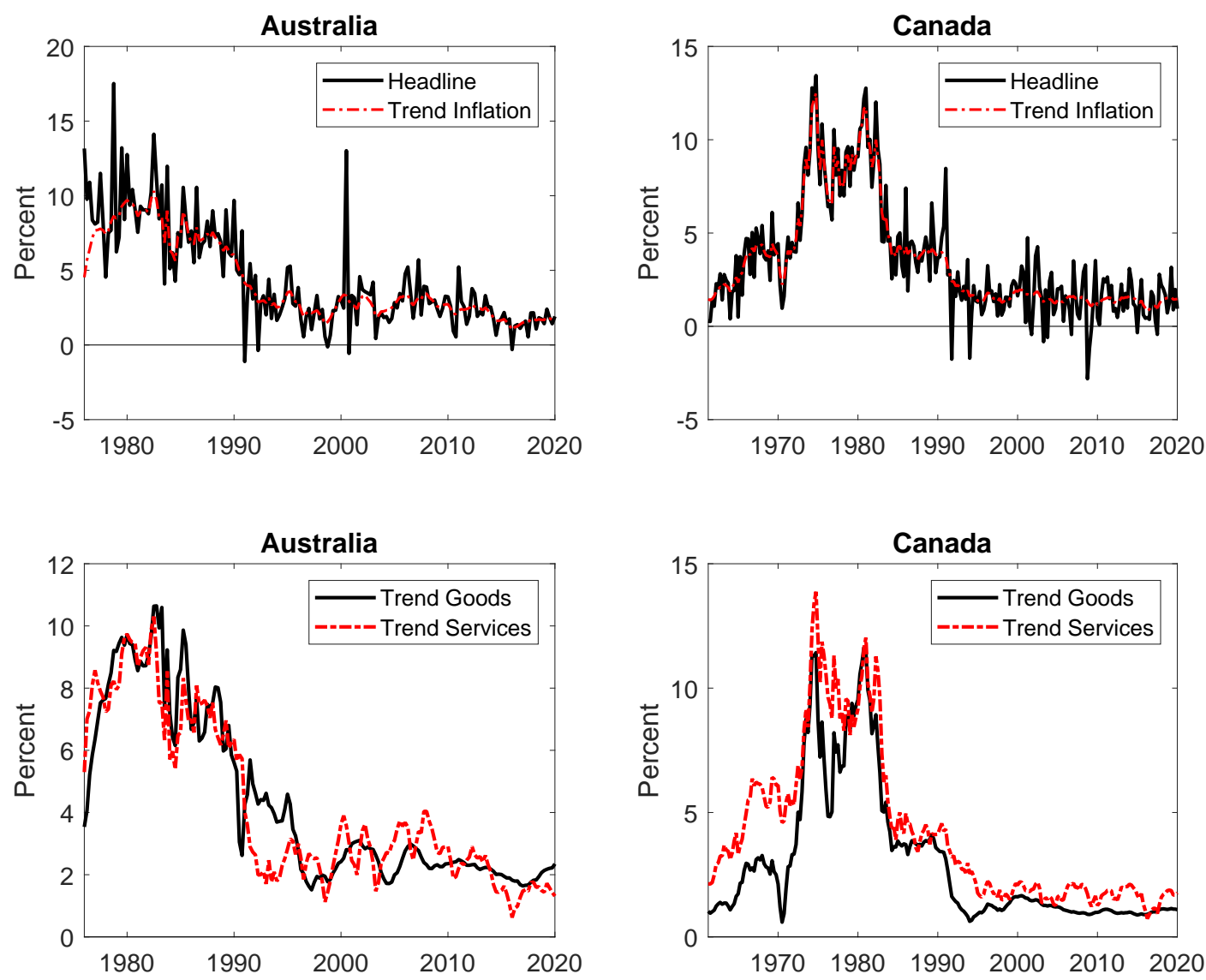

Notes: Trend inflation estimates are obtained as the posterior median estimates from our model. All units are in terms of annualized quarter-on-quarter inflation.

Second, as small open economies that do not have much pricing power in the international goods market, the difference in the inflation dynamics of goods and services sectors reflecting the traded-nontraded goods dichotomy is perhaps much sharper for both Australia and Canada. This feature at least allows us to form a firmer view on the globalization of inflation hypothesis through the lens of our framework.

Figure 9 presents both aggregate and sector-specific inflation estimates for Australia and Canada. We also plot the annualized quarter-on-quarter inflation rate alongside the aggregate trend inflation estimate for the sake of comparison. Note that the sector-specific and aggregate inflation rates are constructed from the consumption deflators implied by 
the household consumption expenditure in the national accounts, to be consistent with the analysis for the US. Unsurprisingly, aggregate trend inflation is a smoother version of headline inflation. Even so, we once again find that while trend inflation tracked headline inflation more closely, especially during the 1970s, trend inflation is a lot smoother since the 1990s. When we turn to the sector-specific trend inflation estimates in the bottom two panels, we observe that while both trend services and trend goods inflation were reasonably volatile in the 1970s, since the 1990s, trend goods inflation has become less volatile. Trend services is more volatile than trend goods in an absolute sense since the 1990s. Even so, trend services inflation since the 1990s is less volatile when compared to its volatility before the 1990s. We also note that, while the estimated aggregate and sector-specific trend inflation dynamics in Australia and Canada have features that are broadly in line with the US, Canada's trend inflation dynamics do mirror the US a lot more closely. This could reflect their geographic proximity or the fact that Canadian trade is very integrated with the US. For example, even though we find that the volatility of goods inflation has fallen markedly in both Australia and Canada, trend goods volatility in Australia still features some variation, whereas trend goods inflation in Canada - similar to the US - appears to exhibit very little since around the mid-1990s. Their aggregate and sector-specific trend inflation volatility do not differ relative to the US.

Figure 10 presents the decomposition of the variance from the innovations to trend inflation for both Australia and Canada using Equation (13). Similar to the US, we find that while all three components contributed to the variation in aggregate trend inflation during the 1980s, and even part of the 1990s for Australia, variation in aggregate trend inflation in both Australia and Canada since around the 1990s has been dominated by variation in trend services inflation. In particular, this result is once again driven by the goods and covariance component no longer contributing to the overall variation in aggregate trend inflation.

That we find that our key result of variation in aggregate trend inflation is now dominated by only the services sector for both Australia and Canada suggests that our key result is not a US-specific phenomenon. As we mentioned at the outset of the section, both Australia and Canada are small open economies which are, more so than the US, price takers in the international goods market, and their services sector likely reflect the domestic economic 
Figure 10: Decomposition of Volatility of Aggregate Trend Inflation for Australia and Canada
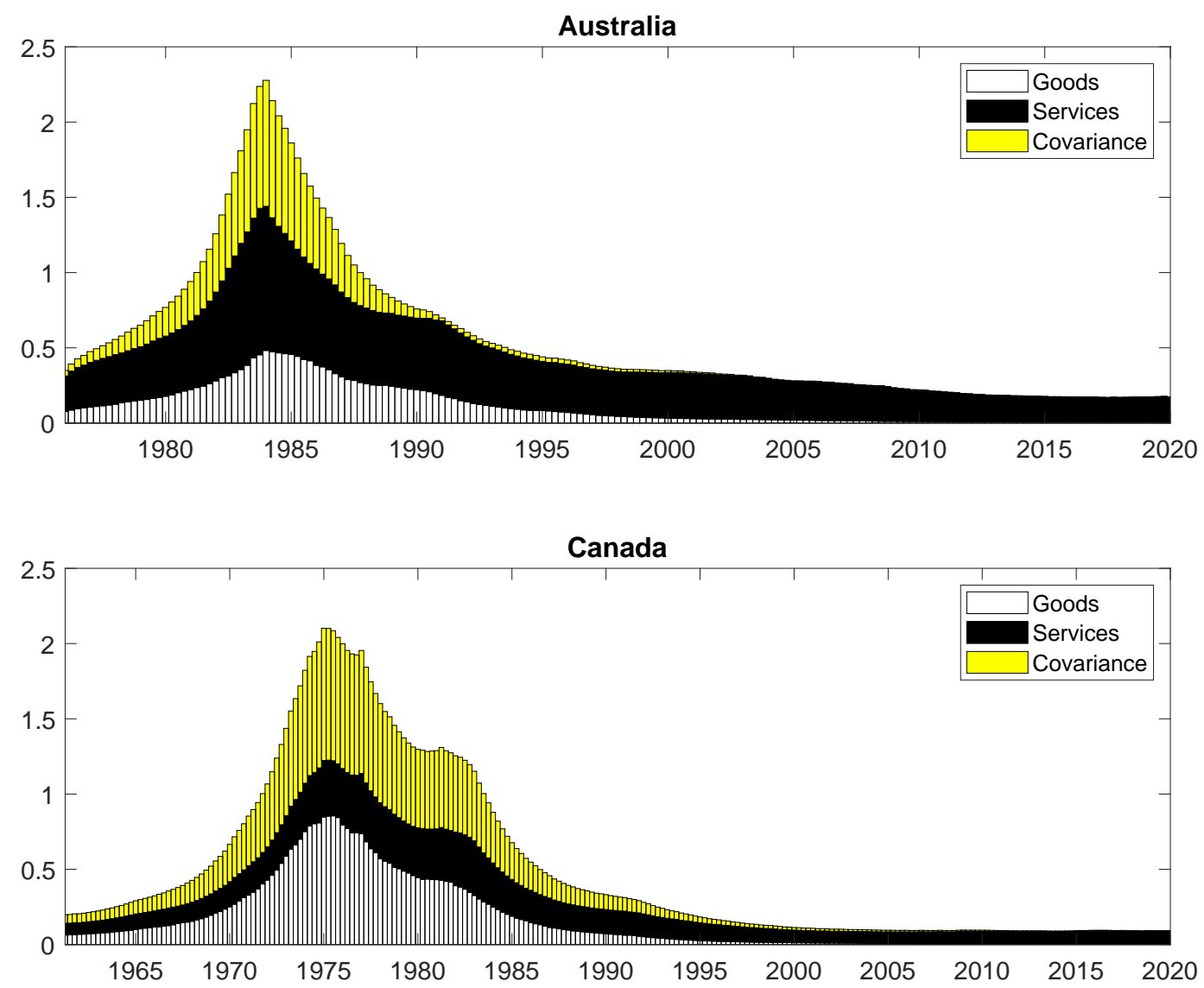

Notes: Trend inflation is in units of annualized quarter-on-quarter inflation. Goods, services and covariance refer to the decomposition of the components of aggregate trend inflation as presented in Equation (13).

environment. That we find that the variation in aggregate trend inflation is dominated by trend services inflation in two small open economies such as Australia and Canada would further support that in terms of viewing the globalization of inflation hypothesis, variation in goods inflation largely reflects transitory noise for overall trend inflation.

We nonetheless accept that the distinction between what is traded and non-traded as corresponding to the goods and services split may be too crude an approximation. Furthermore, whether a good or a service is, in reality, regarded as traded is less likely an absolute, but more likely a matter of degree, where each good and service possesses both a traded and non-traded component. Nevertheless, to the extent that our crude approximation of 
the goods and services split reflects the traded and non-traded dichotomy, our suggestive evidence is that variation in trend inflation is still largely non-traded, and thus domestically determined. Such results are consistent with Kamber and Wong (2020), who find foreign shocks explain very little of the variance to trend inflation in a group of developed and emerging market economies. ${ }^{23}$

\section{Conclusion}

We developed an empirical two-sector model of trend inflation to understand the role of the goods and services sectors as drivers of trend inflation. Our main finding is that variation in aggregate trend inflation is now entirely dominated by that in services inflation. This is a relatively recent occurrence, as before the 1990s both the goods and services sectors contributed to variations in aggregate trend inflation. Our core result is driven by two changes in sector-specific inflation dynamics. First, the variance of trend goods inflation has fallen sharply compared with the period of the Great Inflation in the 1970s, featuring little to no volatility since around the 1990s. Second, while the Great Inflation in the 1970s featured a moderate correlation between innovations to trend goods and trend services inflation, this correlation essentially disappeared during the 1990s.

We document two further results when extending our analysis. First, we rule out energy prices as a possible driver of our results. Instead, we find suggestive evidence consistent with monetary policy as a possible explanation for our results. Second, we document similar inflation dynamics when we extend our analysis to Australia and Canada. Since Australia and Canada are small open economies, and thus likely candidates for viewing the goods and services split as reflecting their traded and non-traded sectors, our result on the variation in trend inflation now being dominated by trend services supports the notion that trend inflation is largely driven by the domestic sector.

Finally, we document one area for future work. Throughout this paper, we have assumed the weights of the services and goods sector are determined exogenously. While this is prob-

\footnotetext{
${ }^{23}$ We note that the sample for Australia and Canada that Kamber and Wong (2020) use does not include the Great Inflation, which means our results in the latter part of our sample are entirely consistent with their work.
} 
ably an innocuous assumption within our context, it does not explain why the services sector has risen in terms of its importance. To illustrate, using a multi-sector growth model, Ngai and Pissarides (2007) show that, when two sectors are complements, the sector with higher productivity growth will have lower sectoral inflation and a lower share of nominal expenditures than the other sector with lower productivity growth. Such a theoretical prediction is consistent with the dynamics of the sectoral trends we estimated and the historical nominal expenditure shares used in our exercise. In other words, trend services inflation has been higher than trend goods inflation, while the nominal share of expenditure on services has increased over time. An avenue for endogenizing changes in the weights within an empirical setting could be via the modeling approaches developed by Kim, Piger and Startz (2008) or Leiva-León and Uzeda (2020).

\section{References}

Andolfatto, David, and Andrew Spewak. 2019. "Understanding Lowflation." Federal Reserve of St. Louis Review, 101(1): 1-26.

Ascari, Guido, and Argia M Sbordone. 2014. "The Macroeconomics of Trend Inflation." Journal of Economic Literature, 52(3): 679-739.

Beveridge, Stephen, and Charles R Nelson. 1981. "A New Approach to Decomposition of Economic Time Series into Permanent and Transitory Components with Particular Attention to Measurement of the Business Cycle." Journal of Monetary Economics, 7(2): 151-174.

Bianchi, Francesco, and Andrea Civelli. 2015. "Globalization and Inflation: Evidence from a Time Varying VAR." Review of Economic Dynamics, 18(2): 406-433.

Boivin, Jean, and Marc P Giannoni. 2006. "Has Monetary Policy Become More Effective?" The Review of Economics and Statistics, 88(3): 445-462.

Borio, Claudio E. V., and Andrew Filardo. 2007. "Globalisation and inflation: New crosscountry evidence on the global determinants of domestic inflation."

Bryan, Michael F., and Stephen G. Cecchetti. 1994. "Measuring Core Inflation." In Monetary Policy. National Bureau of Economic Research, Inc. 
Cecchetti, Stephen G., Peter Hooper, Bruce C. Kasman, Kermit L. Schoenholtz, and Mark W. Watson. 2007. "Understanding the Evolving Inflation Process." US Monetary Policy Forum.

Chan, Joshua C. C., Gary Koop, and Simon M. Potter. 2013. "A New Model of Trend Inflation." Journal of Business \& Economic Statistics, 31(1): 94-106.

Chan, Joshua CC, and Ivan Jeliazkov. 2009. "Efficient Simulation and Integrated Likelihood Estimation in State Space Models." International Journal of Mathematical Modelling and Numerical Optimisation, 1(1-2): 101-120.

Chan, Joshua C.C., Todd E. Clark, and Gary Koop. 2018. "A New Model of Inflation, Trend Inflation, and Long Run Inflation Expectations." Journal of Money, Credit and Banking, 50(1): 5-53.

Clark, Todd E. 2004. "An evaluation of the decline in goods inflation." Economic Review, , (Q II): 19-51.

Cogley, Timothy. 2002. "A Simple Adaptive Measure of Core Inflation." Journal of Money, Credit and Banking, 34(1): 94-113.

Cogley, Timothy, Giorgio E. Primiceri, and Thomas J. Sargent. 2010. "Inflation-Gap Persistence in the US." American Economic Journal: Macroeconomics, 2(1).

Coibion, Olivier, and Yuriy Gorodnichenko. 2011. "Monetary Policy, Trend Inflation, and the Great Moderation: An Alternative Interpretation." American Economic Review, 101(1): 341-370.

Draghi, Mario. 2015. "Global and domestic inflation." Speech at Economic Club of New York, 4 December 2015.

Eo, Yunjong. 2016. "Structural Changes in Inflation Dynamics: Multiple Breaks at Different Dates for Different Parameters." Studies in Nonlinear Dynamics \&5 Econometrics, 20(3): 211-231.

Foerster, Andrew T, Pierre-Daniel G Sarte, and Mark W Watson. 2011. "Sectoral versus Aggregate Shocks: A Structural Factor Analysis of Industrial Production." Journal of Political Economy, 119(1): 1-38.

Garin, Julio, Michael J Pries, and Eric R Sims. 2018. "The Relative Importance of Aggregate and Sectoral Shocks and the Changing Nature of Economic Fluctuations." American Economic Journal: Macroeconomics, 10(1): 119-148. 
Hwu, Shih-tang, and Chang-jin Kim. 2019. "Estimating trend inflation based on unobserved components model: is it correlated with the inflation gap?" Journal of Money, Credit and Banking, 51(8): 2305-2319.

Ireland, Peter N. 2007. "Changes in the Federal Reserve's Inflation Target: Causes and Consequences." Journal of Money, credit and Banking, 39(8): 1851-1882.

Kamber, Günes, and Benjamin Wong. 2020. "Global factors and trend inflation." Journal of International Economics, 122: 103265.

Kamin, Steven B., Mario Marazzi, and John W. Schindler. 2006. "The Impact of Chinese Exports on Global Import Prices." Review of International Economics, 14(2): 179-201.

Kim, C., J. Piger, and R. Startz. 2008. "Estimation of Markov Regime-Switching Regression Models with Endogenous Switching." Journal of Econometrics, 143: 263-273.

Kozicki, Sharon, and Peter A Tinsley. 2001. "Shifting Endpoints in the Term Structure of Interest Rates." Journal of monetary Economics, 47(3): 613-652.

Leiva-León, Danilo, and Luis Uzeda. 2020. "Endogenous Time Variation in Vector Autoregressions." Bank of Canada Staff Working Papers 20-16.

Luciani, Matteo. 2020. "Common and Idiosyncratic Inflation." Board of Governors of the Federal Reserve System (U.S.) Finance and Economics Discussion Series 2020-024.

Mertens, Elmar. 2016. "Measuring the Level and Uncertainty of Trend Inflation." The Review of Economics and Statistics, 98(5): 950-967.

Mishkin, Frederic S. 2007. "Headline versus Core Inflation in the Conduct of Monetary Policy." Business Cycles, International Transmission and Macroeconomic Policies Conference, HEC Montréal, Montréal, Canada, October 20, 2007.

Ngai, L Rachel, and Christopher A Pissarides. 2007. "Structural Change in a Multisector Model of Growth." American Economic Review, 97(1): 429-443.

Omori, Yasuhiro, Siddhartha Chib, Neil Shephard, and Jouchi Nakajima. 2007. "Stochastic Volatility with Leverage: Fast and Efficient Likelihood Inference." Journal of Econometrics, 140(2): 425-449.

Orphanides, Athanasios. 2004. "Monetary Policy Rules, Macroeconomic Stability, and Inflation: A View from the Trenches." Journal of Money, Credit and Banking, 151-175.

Peach, Richard, Robert W. Rich, and Alexis Antoniades. 2004. "The historical and recent behavior of goods and services inflation." Economic Policy Review, , (Dec): 19-31. 
Reis, Ricardo, and Mark W. Watson. 2010. "Relative Goods' Prices, Pure Inflation, and the Phillips Correlation." American Economic Journal: Macroeconomics, 2(3): 128-157.

Stock, James H., and Mark W. Watson. 2007. "Why Has U.S. Inflation Become Harder to Forecast?" Journal of Money, Credit and Banking, 39(s1): 3-33.

Stock, James H., and Mark W. Watson. 2016. "Core Inflation and Trend Inflation." The Review of Economics and Statistics, 98(4): 770-784.

Tallman, Ellis W., and Saeed Zaman. 2017. "Forecasting inflation: Phillips curve effects on services price measures." International Journal of Forecasting, 33(2): 442-457.

Wolman, Alexander L. 2011. "The Optimal Rate of Inflation with Trending Relative Prices." Journal of Money, Credit and Banking, 43: 355-384. 


\title{
Online Appendix to \\ Understanding Trend Inflation Through the Lens of the Goods and Services Sectors *
}

\author{
Yunjong $\mathrm{Eo}^{\dagger}$ \\ Luis Uzeda $a^{\ddagger}$ \\ Benjamin Wong ${ }^{\S}$ \\ Korea University \\ Bank of Canada \\ Monash University
}

\section{Contents}

A1Estimation Details $\quad 1$

A1.1 Priors . . . . . . . . . . . . . . . . . . . . . . . 4

A1.2 Posterior Sampler . . . . . . . . . . . . . . . . . . . 5

A1.2.1 State Simulation . . . . . . . . . . . . . . . . . . 6

A1.2.2 Parameter Sampling . . . . . . . . . . . . . . 13

A2Model Comparison $\quad 15$

A2.1 Extensions to the Baseline MCMC Algorithm . . . . . . . . . . . . . 16

A3Additional Results from Two-Sector UC-SV 19

A3.1 Credible Sets of Trend Inflation Estimate . . . . . . . . . . . . . . 19

A3.2 Comparison Relative to Stock and Watson (2007) UUC-SV . . . . . . . . . 19

\section{A4Details on Implementing Identification of the Pure Inflation Component 24}

${ }^{*}$ The views expressed are those of the authors and do not necessarily reflect the position of the Bank of Canada. All remaining errors are ours.

${ }^{\dagger}$ Yunjong Eo: Department of Economics, Korea University, Seoul 02841, South Korea; Tel: +82 23290 2212; Email: yunjongeo@korea.ac.kr

${ }^{\ddagger}$ Luis Uzeda: Bank of Canada, 234 Wellington Ave W, Ottawa, ON, K1A 0H9, Canada; Email: luzedagarcia@bank-banque-canada.ca

$\S$ Benjamin Wong: Department of Econometrics and Business Statistics, Monash University, Caulfield East, VIC 3145, Australia; Email: benjamin.wong@monash.edu 
A5.1 Constructing the Goods Ex-Energy and Services Ex-Energy Series . . . . . 26

A5.2 Other Results with Ex-Energy Data . . . . . . . . . . . . . . 27

A6 Australia and Canada Data $\quad 29$

A6.1 Construction of Goods and Services Deflators for Australia and Canada . . . 29

A6.2 Other Australia and Canada Results . . . . . . . . . . . . . . . . . . . 33 


\section{A1 Estimation Details}

Estimation of the states in our model is carried out using precision sampling methods as in Chan and Jeliazkov (2009). Under this approach, the signal extraction exercise operates on a parametrization of the model in Section 2.1 where all observables and state variables are stacked over $t=1, \ldots, T$. For convenience, below we reproduce the stacked representation of the two-sector UC model that we base our posterior sampler upon. Formally, we have

$$
\begin{aligned}
& \mathbf{y}=\boldsymbol{\tau}+\boldsymbol{\zeta} \\
& \mathbf{L}_{\tau} \boldsymbol{\tau}=\boldsymbol{\tau}_{0}+\mathbf{u}^{\tau} \\
& \mathbf{L}_{h} \mathbf{h}=\mathbf{h}_{0}+\mathbf{u}^{h} \\
& \mathbf{L}_{\gamma} \gamma=\gamma_{0}+\mathbf{u}^{\gamma} \\
& {\left[\begin{array}{c}
\boldsymbol{\zeta} \\
\mathbf{u}^{\tau} \\
\mathbf{u}^{h} \\
\mathbf{u}^{\gamma}
\end{array}\right] \sim \mathcal{N}\left(\left[\begin{array}{l}
0 \\
0 \\
0 \\
0
\end{array}\right],\left[\begin{array}{cccc}
\Sigma_{\zeta} & 0 & 0 & 0 \\
0 & \Sigma_{\tau} & 0 & 0 \\
0 & 0 & \Sigma_{h} & 0 \\
0 & 0 & 0 & \Sigma_{\gamma}
\end{array}\right]\right) }
\end{aligned}
$$


where:

$$
\begin{aligned}
& \underset{(2 T \times 1)}{\mathbf{y}}=\left[\begin{array}{c}
\left(\pi_{1}^{G}, \cdots, \pi_{T}^{G}\right)^{\prime} \\
\left(\pi_{1}^{S}, \cdots, \pi_{T}^{S}\right)^{\prime}
\end{array}\right], \underset{(2 T \times 1)}{\boldsymbol{\tau}}=\left[\begin{array}{c}
\left(\tau_{1}^{G}, \cdots, \tau_{T}^{G}\right)^{\prime} \\
\left(\tau_{1}^{S}, \cdots, \tau_{T}^{S}\right)^{\prime}
\end{array}\right], \underset{(4 T \times 1)}{\mathbf{h}}=\left[\begin{array}{c}
\left(h_{1}^{\zeta^{G}}, \cdots, h_{T}^{\zeta^{G}}\right)^{\prime} \\
\left(h_{1}^{\zeta^{S}}, \cdots, h_{T}^{\zeta^{S}}\right)^{\prime} \\
\left(h_{1}^{\tau^{G}}, \cdots, h_{T}^{\tau^{G}}\right)^{\prime} \\
\left(h_{1}^{\tau^{S}}, \cdots, h_{T}^{\tau^{S}}\right)^{\prime}
\end{array}\right], \\
& \underset{(2 T \times 1)}{\gamma}=\left[\begin{array}{c}
\left(\gamma_{1}^{\zeta}, \cdots, \gamma_{T}^{\zeta}\right)^{\prime} \\
\left(\gamma_{1}^{\tau}, \cdots, \gamma_{T}^{\tau}\right)^{\prime}
\end{array}\right] \underset{(2 T \times 1)}{\boldsymbol{\zeta}}=\left[\begin{array}{c}
\left(\zeta_{1}^{G}, \cdots, \zeta_{T}^{G}\right)^{\prime} \\
\left(\zeta_{1}^{S}, \cdots, \zeta_{T}^{S}\right)^{\prime}
\end{array}\right] \underset{(2 T \times 1)}{\mathbf{u}^{\tau}}=\left[\begin{array}{c}
\left(u_{1}^{\tau^{G}}, \cdots, u_{T}^{\tau^{G}}\right)^{\prime} \\
\left(u_{1}^{\tau^{S}}, \cdots, u_{T}^{\tau^{S}}\right)^{\prime}
\end{array}\right] \\
& \underset{(4 T \times 1)}{\mathbf{u}^{h}}\left[\begin{array}{c}
\left(u_{1}^{h^{\zeta^{G}}}, \cdots, u_{T}^{h^{\zeta^{G}}}\right)^{\prime} \\
\left(u_{1}^{h^{\zeta^{S}}}, \cdots, u_{T}^{h^{\zeta^{S}}}\right)^{\prime} \\
\left(u_{1}^{h^{\tau G}}, \cdots, u_{T}^{h^{\tau G}}\right)^{\prime} \\
\left(u_{1}^{h^{\tau S}}, \cdots, u_{T}^{h^{\tau S}}\right)^{\prime}
\end{array}\right], \underset{(2 T \times 1)}{\underset{\mathbf{u}^{\gamma}}{\gamma}}=\left[\begin{array}{c}
\left(u_{1}^{\gamma^{\zeta}}, \cdots, u_{T}^{\gamma^{\zeta}}\right)^{\prime} \\
\left(u_{1}^{\gamma^{\tau}}, \cdots, u_{T}^{\gamma^{\tau}}\right)^{\prime}
\end{array}\right]
\end{aligned}
$$

Initialization conditions are treated as additional parameters in our MCMC algorithm and collected as follows:

$$
\underset{(2 T \times 1)}{\boldsymbol{\tau}_{0}}=\left[\begin{array}{c}
\tau_{0}^{G} \\
\tau_{0}^{S}
\end{array}\right] \otimes\left[\begin{array}{c}
1 \\
0 \\
\vdots \\
0
\end{array}\right], \underset{(4 T \times 1)}{\mathbf{h}_{0}}=\left[\begin{array}{c}
h_{0}^{\zeta^{G}} \\
h_{0}^{\zeta^{S}} \\
h_{0}^{\tau G} \\
h_{0}^{\tau S}
\end{array}\right] \otimes\left[\begin{array}{c}
1 \\
0 \\
\vdots \\
0
\end{array}\right], \underset{(2 T \times 1)}{\gamma_{0}}=\left[\begin{array}{c}
\gamma_{0}^{\zeta} \\
\gamma_{0}^{\tau}
\end{array}\right] \otimes\left[\begin{array}{c}
1 \\
0 \\
\vdots \\
0
\end{array}\right]
$$


where $\otimes$ denotes the Kronecker operator. Next, the matrices pre-multiplying the vector of states are sparse structures defined as follows:

$$
\begin{aligned}
\underset{(2 T \times 2 T)}{\mathbf{L}_{\tau}}=\mathbf{L}_{\gamma}=\left[\begin{array}{cc}
\mathbf{H} & \mathbf{0} \\
\mathbf{0} & \mathbf{H}
\end{array}\right], \underset{(4 T \times 4 T)}{\mathbf{L}_{h}}=\left[\begin{array}{cccc}
\mathbf{H} & \mathbf{0} & \mathbf{0} & \mathbf{0} \\
\mathbf{0} & \mathbf{H} & \mathbf{0} & \mathbf{0} \\
\mathbf{0} & \mathbf{0} & \mathbf{H} & \mathbf{0} \\
\mathbf{0} & \mathbf{0} & \mathbf{0} & \mathbf{H}
\end{array}\right] \text {, such that } \\
\underset{(T \times T)}{\mathbf{H}}=\left[\begin{array}{ccccc}
1 & 0 & 0 & \cdots & 0 \\
-1 & 1 & 0 & \cdots & 0 \\
0 & -1 & 1 & & \vdots \\
\vdots & \vdots & \ddots & \ddots & \\
0 & 0 & \cdots & -1 & 1
\end{array}\right]
\end{aligned}
$$

Finally, each covariance matrix in the system is given by

$$
\underset{(2 T \times 2 T)}{\boldsymbol{\Sigma}_{\ell}}=\left[\begin{array}{cccccc}
\exp \left(h_{1}^{\ell^{G}}\right) & \cdots & 0 & \gamma_{1}^{\ell} \exp \left(h_{1}^{\ell^{G}}\right) & \cdots & 0 \\
\vdots & \ddots & \vdots & \vdots & \ddots & \vdots \\
0 & \cdots & \exp \left(h_{T}^{\ell^{G}}\right) & 0 & \cdots & \gamma_{T}^{\ell} \exp \left(h_{T}^{\ell^{G}}\right) \\
\gamma_{1}^{\ell} \exp \left(h_{1}^{\ell^{G}}\right) & \cdots & 0 & \gamma_{1}^{\ell^{2}} \exp \left(h_{1}^{\ell^{G}}\right)+\exp \left(h_{1}^{\ell^{S}}\right) & \cdots & 0 \\
\vdots & \ddots & \vdots & \vdots & \ddots & \vdots \\
0 & \cdots & \gamma_{T}^{\ell} \exp \left(h_{T}^{\ell^{G}}\right) & 0 & \cdots & \gamma_{T}^{\ell^{2}} \exp \left(h_{T}^{\ell^{G}}\right)+\exp \left(h_{T}^{\ell^{S}}\right)
\end{array}\right],
$$

for $\ell=\zeta$ and $\tau$,

$$
\begin{aligned}
\underset{(4 T \times 4 T)}{\boldsymbol{\Sigma}_{h}}= & {\left[\begin{array}{cccc}
\sigma_{h^{\zeta}}^{2} I_{T} & \mathbf{0} & \mathbf{0} & \mathbf{0} \\
\mathbf{0} & \sigma_{h \zeta^{S}}^{2} I_{T} & \mathbf{0} & \mathbf{0} \\
\mathbf{0} & \mathbf{0} & \sigma_{h^{\tau G}}^{2} I_{T} & \mathbf{0} \\
\mathbf{0} & \mathbf{0} & \mathbf{0} & \sigma_{h^{\tau G}}^{2} I_{T}
\end{array}\right], } \\
\underset{(2 T \times 2 T)}{\boldsymbol{\Sigma}_{\gamma}} & =\left[\begin{array}{cc}
\sigma_{\gamma^{\zeta}}^{2} I_{T} & \mathbf{0} \\
\mathbf{0} & \sigma_{\gamma^{\tau}}^{2} I_{T}
\end{array}\right],
\end{aligned}
$$


where $I_{T}$ denotes a $T$ dimensional identity matrix.

\section{A1.1 Priors}

There are three blocks of model parameters in our baseline Two-Sector UC model, i.e.:

$$
\mathrm{z}_{0}=\left[\begin{array}{c}
\tau_{0}^{G} \\
\tau_{0}^{S} \\
h_{0}^{\zeta^{G}} \\
h_{0}^{\zeta^{S}} \\
h_{0}^{\tau G} \\
h_{0}^{\tau S} \\
\gamma_{0}^{\zeta} \\
\gamma_{0}^{\tau}
\end{array}\right], \Omega_{h}=\operatorname{diag}\left(\sigma_{h^{G}}^{2}, \sigma_{h^{\zeta}}^{2}, \sigma_{h^{\tau G}}^{2}, \sigma_{h^{\tau G}}^{2}\right), \text { and } \Omega_{\gamma}=\operatorname{diag}\left(\sigma_{\gamma^{\zeta}}^{2}, \sigma_{\gamma^{\tau}}^{2}\right)
$$

We assume standard independent priors for each of these three blocks of parameters. More precisely:

$$
\begin{aligned}
\mathrm{z}_{0} & \sim \mathcal{N}\left(\widehat{\mathrm{z}_{0}}, \Sigma_{z_{0}}\right) \\
\sigma_{\gamma^{\ell}}^{2} & \sim \mathcal{I} \mathcal{G}\left(\nu_{\gamma^{\ell}}, S_{\gamma^{\ell}}\right) \\
\sigma_{h^{\ell^{i}}}^{2} & \sim \mathcal{I} \mathcal{G}\left(\nu_{h^{\ell^{i}}}, S_{h^{\ell^{i}}}\right) \text { for } \ell=\zeta \text { and } \tau \text { and } i=G \text { and } S .
\end{aligned}
$$

The choice of prior densities adopted in this paper are in line with previous studies on trend inflation that also rely on UC-SV models (e.g., see Chan, Koop and Potter, 2013, 2016). We 
calibrate our priors as follows:

$$
\left\{\begin{array}{l}
\widehat{\mathrm{z}_{0}}=\left(\pi_{1}^{G}, \pi_{1}^{S}, 0, \cdots, 0\right)^{\prime}, \\
\Sigma_{z_{0}}=100 \times I_{8} \\
\nu_{\gamma^{\ell}}=\frac{T}{10} \\
S_{\gamma^{\ell}}=0.1^{2}\left(\nu_{\gamma^{\ell}}-1\right) \\
\nu_{h^{\ell^{i}}}=\frac{T}{10} \\
S_{h^{\ell^{i}}}=0.17^{2}\left(\nu_{h^{\ell}}-1\right) \text { for } \ell=\zeta \text { and } \tau \text { and } i=G \text { and } S .
\end{array}\right.
$$

Notably, the values assigned to $\Sigma_{z_{0}}$ and to the shape parameters of the inverse-gamma densities $-\nu_{\gamma^{\ell}}$ and $\nu_{h^{\ell^{i}}}$ - reflect relatively uninformative priors.

\section{A1.2 Posterior Sampler}

Now let $\mathbf{Z}=\left\{\boldsymbol{\tau}, \mathbf{h}, \mathrm{z}_{0}, \Omega_{h}, \Omega_{\gamma}\right\}$ denote the set of states and parameters in our TwoSector UC model, where notation $\mathbf{Z}_{-j}$ represents all elements in $\mathbf{Z}$ except for $j$. An MCMC algorithm for estimating such a model entails sequentially sampling from the following conditional posterior distributions:

(1) $f\left(\boldsymbol{\tau} \mid \mathbf{y}, \mathbf{Z}_{-\boldsymbol{\tau}}\right)$,

(2) $f\left(\mathbf{h} \mid \mathbf{y}, \mathbf{Z}_{-\mathbf{h}}\right)$,

(3) $f\left(\gamma \mid \mathbf{y}, \mathbf{Z}_{-\gamma}\right)$,

(4) $f\left(\mathrm{z}_{0} \mid \mathbf{y}, \mathbf{Z}_{-\mathrm{z}_{0}}\right)$,

(5) $f\left(\Omega_{h} \mid \mathbf{y}, \mathbf{Z}_{-\boldsymbol{\Omega}_{h}}\right)$,

(6) $f\left(\Omega_{\gamma} \mid \mathbf{y}, \mathbf{Z}_{-\Omega_{\gamma}}\right)$.

Steps 1 through 3 above denote the state simulation block in our MCMC - i.e. drawing the time-varying parameters. The remaining steps correspond to drawing from the full conditional posteriors for the fixed parameters. Hence, we refer to steps 4 through 6 as the parameter sampling block in our algorithm. Below, we describe these two main blocks in 
greater detail.

\section{A1.2.1 State Simulation}

\section{- Sampling $\tau$}

First note that Equations (A1) and (A2) denote a linear Gaussian state space representation. Therefore, standard multivariate regression results (see, e.g., Koop, Poirier and Tobias (2007)) can be used to show that the conditional posterior for $\boldsymbol{\tau}$ is also Gaussian. More precisely, we have:

$$
\boldsymbol{\tau} \mid \mathbf{y}, \mathbf{Z}_{-\boldsymbol{\tau}} \sim \mathcal{N}\left(\overline{\mathbf{d}}_{\tau}, \overline{\mathbf{D}}_{\tau}\right), \text { where }\left\{\begin{array}{l}
\overline{\mathbf{d}}_{\tau}=\overline{\mathbf{D}}_{\tau}\left(\boldsymbol{\Sigma}_{\zeta}^{-1} \mathbf{y}+\mathbf{L}_{\tau}^{\prime} \boldsymbol{\Sigma}_{\tau}^{-1} \boldsymbol{\tau}_{0}\right) \\
\overline{\mathbf{D}}_{\tau}=\left(\boldsymbol{\Sigma}_{\zeta}^{-1}+\mathbf{L}_{\tau}^{\prime} \boldsymbol{\Sigma}_{\tau}^{-1} \mathbf{L}_{\tau}\right)^{-1}
\end{array}\right.
$$

As shown in the second equation in (A9), sampling $\boldsymbol{\tau}$ entails inverting the $2 T \times 2 T$ matrix $\left(\boldsymbol{\Sigma}_{\zeta}^{-1}+\mathbf{L}_{\tau}^{\prime} \boldsymbol{\Sigma}_{\tau}^{-1} \mathbf{L}_{\tau}\right)$ to construct the covariance matrix $\overline{\mathbf{D}}_{\tau}$. We do so by applying precision sampling techniques proposed in Chan and Jeliazkov (2009), which provides an efficient way to expedite computation. ${ }^{1}$

To illustrate how we employ their algorithm, we introduce the following notation: given a lower triangular $2 T \times 2 T$ non-singular matrix $\mathbf{C}$ and a $2 T \times 1$ vector $\mathbf{b}$, let $\mathbf{C} \backslash \mathbf{b}$ denote the unique solution to the triangular system $\mathbf{C x}=\mathbf{b}$ obtained by forward substitution, i.e., $\mathbf{x}=\mathbf{C} \backslash \mathbf{b}=\mathbf{C}^{-1} \mathbf{b}$. Sampling $\boldsymbol{\tau}$ is then conducted by following the four operations below:

$$
\begin{aligned}
& \text { (1) } \operatorname{Chol}\left(\overline{\mathbf{D}}_{\tau}^{-1}\right)=\mathbf{C C}^{\prime}, \\
& \text { (2) } \mathbf{x}=\mathbf{C} \backslash\left(\boldsymbol{\Sigma}_{\zeta}^{-1} \mathbf{y}+\mathbf{L}_{\tau}^{\prime} \boldsymbol{\Sigma}_{\tau}^{-1} \boldsymbol{\tau}_{0}\right), \\
& \text { (3) } \overline{\mathbf{d}}_{\tau}=\mathbf{C}^{\prime} \backslash \mathbf{x}, \\
& \text { (4) } \boldsymbol{\tau}=\overline{\mathbf{d}}_{\tau}+\mathbf{C}^{\prime} \backslash \mathbf{e} \quad \mathbf{e} \sim \mathcal{N}\left(\mathbf{0}, I_{2 T}\right) .
\end{aligned}
$$

The first step describes the Cholesky decomposition of the inverse covariance (or precision) matrix $\overline{\mathbf{D}}_{\tau}^{-1}$. Step 2 requires solving a triangular system by forward substitution, given that

\footnotetext{
${ }^{1} \mathrm{~A}$ detailed comparison between precision- and Kalman filter-based techniques for state simulation pointing out the benefits of the former over the latter - can be found in McCausland, Miller and Pelletier (2011).
} 
$\mathbf{C}$ is a lower triangular matrix. Step 3 is analogous to Step 2, except that the solution of the triangular system, $\mathbf{C}^{\prime} \backslash \mathbf{x}$, is now obtained by backward substitution, since $\mathbf{C}^{\prime}$ is an upper triangular matrix. It is then straightforward to see that Steps 2 and 3 combined, by construction, yield:

$\overline{\mathbf{d}}_{\tau}=\mathbf{C}^{\prime-1}\left(\mathbf{C}^{-1}\left(\boldsymbol{\Sigma}_{\zeta}^{-1} \mathbf{y}+\mathbf{L}_{\tau}^{\prime} \boldsymbol{\Sigma}_{\tau}^{-1} \boldsymbol{\tau}_{0}\right)\right)=\left(\mathbf{C C}^{\prime}\right)^{-1}\left(\boldsymbol{\Sigma}_{\zeta}^{-1} \mathbf{y}+\mathbf{L}_{\tau}^{\prime} \boldsymbol{\Sigma}_{\tau}^{-1} \boldsymbol{\tau}_{0}\right)=\overline{\mathbf{D}}_{\tau}^{-1}\left(\boldsymbol{\Sigma}_{\zeta}^{-1} \mathbf{y}+\mathbf{L}_{\tau}^{\prime} \boldsymbol{\Sigma}_{\tau}^{-1} \boldsymbol{\tau}_{0}\right)$

Finally, Step 4 describes an affine transformation of standard normal random vector e which ensures, by definition, that after sampling $\mathbf{e} \sim \mathcal{N}\left(\mathbf{0}, I_{2 T}\right)$, the expression in such a step returns a $2 T \times 1$ random vector $\boldsymbol{\tau} \mid \mathbf{y}, \mathbf{Z}_{-\boldsymbol{\tau}} \sim \mathcal{N}\left(\overline{\mathbf{d}}_{\tau}, \overline{\mathbf{D}}_{\tau}\right)$.

To further expedite computation, in addition to the precision sampling techniques, we resort to an analytical solution - instead of brute-force methods - to obtain the inverse matrices $\boldsymbol{\Sigma}_{\zeta}^{-1}$ and $\boldsymbol{\Sigma}_{\tau}^{-1}$ which are components of $\overline{\mathbf{D}}_{\tau}^{-1}$ and show up in Step 2 of the precision sampling algorithm just described above. In particular, both $\boldsymbol{\Sigma}_{\zeta}^{-1}$ and $\boldsymbol{\Sigma}_{\tau}^{-1}$ can be partitioned into four block-diagonal matrices, as follows:

$$
\Sigma_{\ell}=\left[\begin{array}{cc}
\Sigma_{\ell G} & \Sigma_{\ell G, S} \\
\Sigma_{\ell G, S} & \Sigma_{\ell^{S}}
\end{array}\right]
$$

where:

$$
\begin{aligned}
\boldsymbol{\Sigma}_{\ell^{G}} & =\operatorname{diag}\left(\exp \left(h_{1}^{\ell^{G}}\right), \cdots, \exp \left(h_{T}^{\ell^{G}}\right)\right), \\
\boldsymbol{\Sigma}_{\ell^{S}} & =\operatorname{diag}\left(\gamma_{1}^{\ell^{2}} \exp \left(h_{1}^{\ell^{G}}\right)+\exp \left(h_{1}^{\ell^{S}}\right), \cdots, \gamma_{T}^{\ell^{2}} \exp \left(h_{T}^{\ell^{G}}\right)+\exp \left(h_{T}^{\ell^{S}}\right)\right), \\
\boldsymbol{\Sigma}_{\ell G, S} & =\operatorname{diag}\left(\gamma_{1}^{\ell} \exp \left(h_{1}^{\ell^{G}}\right), \cdots, \gamma_{T}^{\ell} \exp \left(h_{T}^{\ell^{G}}\right)\right), \quad \text { for } \ell=\zeta \text { and } \tau .
\end{aligned}
$$

Matrix inversion results discussed in, e.g., Anderson (1984) yield:

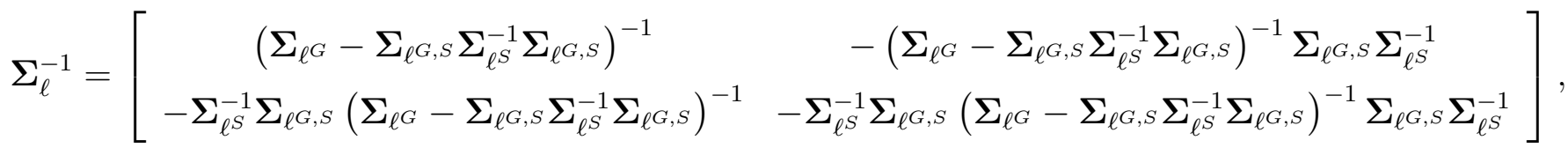

for $\ell=\zeta$ and $\tau$.

It is worth noting that, despite the long algebraic expressions above, constructing each 
block matrix in $\boldsymbol{\Sigma}_{\ell}^{-1}$ only entails operations between diagonal matrices. As a result, an analytical solution for $\Sigma_{\ell}^{-1}$ can be readily derived. ${ }^{2}$ Specifically, we have

$$
\begin{aligned}
& \text { (I) }\left(\boldsymbol{\Sigma}_{\ell^{G}}-\boldsymbol{\Sigma}_{\ell^{G, S}} \boldsymbol{\Sigma}_{\ell^{S}}^{-1} \boldsymbol{\Sigma}_{\ell^{G, S}}\right)^{-1}=\operatorname{diag}\left(\frac{\gamma_{1}^{\ell^{2}} \exp \left(h_{1}^{\ell^{G}}\right)+\exp \left(h_{1}^{\ell^{S}}\right)}{\exp \left(h_{1}^{\ell^{G}}+h_{1}^{\ell^{S}}\right)}, \cdots, \frac{\gamma_{T}^{\ell^{2}} \exp \left(h_{T}^{\ell^{G}}\right)+\exp \left(h_{T}^{\ell^{S}}\right)}{\exp \left(h_{T}^{\ell^{G}}+h_{T}^{\ell^{S}}\right)}\right) \text {, } \\
& \text { (II) }-\Sigma_{\ell^{S}}^{-1} \Sigma_{\ell G, S}\left(\Sigma_{\ell^{G}}-\Sigma_{\ell^{G, S}} \boldsymbol{\Sigma}_{\ell^{S}}^{-1} \boldsymbol{\Sigma}_{\ell^{G, S}}\right)^{-1} \boldsymbol{\Sigma}_{\ell^{G, S}} \boldsymbol{\Sigma}_{\ell^{S}}^{-1}= \\
& =\operatorname{diag}\left(\frac{\gamma_{1}^{\ell^{2}} \exp \left(2 h_{1}^{\ell^{G}}\right)}{\gamma_{1}^{\ell^{2}} \exp \left(2 h_{1}^{\ell^{G}}+h_{1}^{\ell^{S}}\right)+\exp \left(h_{1}^{\ell^{G}}+2 h_{1}^{\ell^{S}}\right)}, \cdots, \frac{\gamma_{T}^{\ell^{2}} \exp \left(2 h_{T}^{\ell^{G}}\right)}{\gamma_{T}^{\ell^{2}} \exp \left(2 h_{T}^{\ell^{G}}+h_{T}^{\ell^{S}}\right)+\exp \left(h_{T}^{\ell^{G}}+2 h_{T}^{\ell^{S}}\right)}\right), \\
& \text { (III) }-\left(\Sigma_{\ell^{G}}-\Sigma_{\ell^{G, S}} \boldsymbol{\Sigma}_{\ell^{S}}^{-1} \boldsymbol{\Sigma}_{\ell G, S}\right)^{-1} \boldsymbol{\Sigma}_{\ell^{G, S}} \boldsymbol{\Sigma}_{\ell^{S}}^{-1}=-\boldsymbol{\Sigma}_{\ell^{S}}^{-1} \boldsymbol{\Sigma}_{\ell G, S}\left(\boldsymbol{\Sigma}_{\ell^{G}}-\boldsymbol{\Sigma}_{\ell^{G, S}} \boldsymbol{\Sigma}_{\ell^{S}}^{-1} \boldsymbol{\Sigma}_{\ell^{G, S}}\right)^{-1}= \\
& =\operatorname{diag}\left(\frac{\gamma_{1}^{\ell^{2}} \exp \left(h_{1}^{\ell^{G}}\right)+\exp \left(h_{1}^{\ell^{S}}\right)}{\exp \left(h_{1}^{\ell^{G}}+h_{1}^{\ell^{S}}\right)}, \cdots, \frac{\gamma_{T}^{\ell} \exp \left(h_{T}^{\ell^{G}}\right)}{\exp \left(h_{T}^{\ell^{G}}+h_{T}^{\ell^{S}}\right)}\right), \quad \text { for } \ell=\zeta \text { and } \tau \text {. }
\end{aligned}
$$

\section{- Sampling $\mathbf{h}$}

To sample log-volatilities $\mathbf{h}$, we combine the auxiliary mixture sampler approach of Omori et al. (2007) with the precision sampling techniques previously described. To this end, note first that we can reexpress equations (A1) and (A2) as

$$
\begin{aligned}
& {\left[\begin{array}{c}
\mathbf{y}-\boldsymbol{\tau} \\
\mathbf{L}_{\tau} \boldsymbol{\tau}-\boldsymbol{\tau}_{0}
\end{array}\right] }=\left[\begin{array}{cc}
\mathbf{A}_{\zeta} & \mathbf{0} \\
\mathbf{0} & \mathbf{A}_{\tau}
\end{array}\right]\left[\begin{array}{cc}
\boldsymbol{\Lambda}_{h^{\zeta}} & \mathbf{0} \\
\mathbf{0} & \boldsymbol{\Lambda}_{h^{\tau}}
\end{array}\right] \varepsilon \\
& {\left[\begin{array}{cc}
\mathbf{A}_{\zeta} & \mathbf{0} \\
\mathbf{0} & \mathbf{A}_{\tau}
\end{array}\right]^{-1}\left[\begin{array}{c}
\mathbf{y}-\boldsymbol{\tau} \\
\mathbf{L}_{\tau} \boldsymbol{\tau}-\boldsymbol{\tau}_{0}
\end{array}\right]=\left[\begin{array}{cc}
\boldsymbol{\Lambda}_{h \zeta} & \mathbf{0} \\
\mathbf{0} & \boldsymbol{\Lambda}_{h^{\tau}}
\end{array}\right] \varepsilon }
\end{aligned}
$$

\footnotetext{
${ }^{2}$ We also conducted estimations where $\boldsymbol{\Sigma}_{\zeta}^{-1}$ and $\boldsymbol{\Sigma}_{\tau}^{-1}$ were constructed via brute-force inversion. Results, as expected, are unchanged, albeit the MCMC sampler takes a bit longer to carry out state simulation.
} 
where

$$
\begin{aligned}
\boldsymbol{\varepsilon} & \sim \mathcal{N}\left(\mathbf{0}, I_{4 T}\right), \\
\mathbf{A}_{\ell} & =\left[\begin{array}{cc}
I_{T} & \mathbf{0} \\
\operatorname{diag}\left(\gamma_{1}^{\ell}, \cdots, \gamma_{T}^{\ell}\right) & I_{T}
\end{array}\right], \\
\boldsymbol{\Lambda}_{h^{\ell}} & =\left[\begin{array}{cc}
\operatorname{diag}\left(\exp \left(h_{1}^{\ell^{G}}\right), \cdots, \exp \left(h_{T}^{\ell^{G}}\right)\right) & \mathbf{0} \\
\mathbf{0} & \operatorname{diag}\left(\exp \left(h_{1}^{\ell^{S}}\right), \cdots, \exp \left(h_{T}^{\ell^{S}}\right)\right)
\end{array}\right]
\end{aligned}
$$

for $\ell=\zeta$ and $\tau$.

Next, let $\widetilde{\mathbf{y}}_{\tau}$ and $\mathbf{v}$ denote the left- and right-hand side of Equation (A11), respectively. Squaring and subsequently taking natural logarithms of each element in the $4 T \times 1$ vectors $\widetilde{\mathbf{y}}_{\tau}$ and $\mathbf{v}$ leads to the following linear state space representation for $\mathbf{h}$ :

$$
\begin{gathered}
\widetilde{\mathbf{y}}_{\tau}^{*}=\mathbf{h}+\widetilde{\mathbf{v}}, \\
\mathbf{L}_{h} \mathbf{h}=\mathbf{h}_{0}+\mathbf{u}^{h},
\end{gathered}
$$

where the state equation for $\mathbf{h}$ comes from (A3). The system above - albeit linear - is no longer Gaussian. More precisely, each entry in $\widetilde{\mathbf{v}}$ follows a log chi-square distribution with one degree of freedom. To bring the state space representation back to Gaussian form, Omori et al. (2007) suggest approximating the distribution of $\widetilde{\mathbf{v}}$ as a mixture of ten Normal densities. ${ }^{3}$ Formally, let $\widetilde{\mathbf{v}}^{*}$ denote such mixture approximation, i.e.:

$$
\widetilde{\mathbf{v}}^{*} \sim p_{1} \mathcal{N}\left(\boldsymbol{\alpha}_{1}, \boldsymbol{\Sigma}_{1}\right)+\cdots+p_{10} \mathcal{N}\left(\boldsymbol{\alpha}_{10}, \boldsymbol{\Sigma}_{10}\right)
$$

where $\boldsymbol{\alpha}_{s}, \boldsymbol{\Sigma}_{s}$ and the component-density probabilities $p_{s}$ for $s=1, \cdots, 10$ are predetermined and given in Table 1 in Omori et al. (2007). Therefore, for a given particular componentdensity $\mathcal{N}\left(\boldsymbol{\alpha}_{s}, \boldsymbol{\Sigma}_{s}\right)$, the state space in (A14)-(A15) can be recast in (conditionally) Gaussian

\footnotetext{
${ }^{3}$ Their approach extends the seven-component auxiliary mixture sampling from Kim, Shephard and Chib (1998).
} 
form as:

$$
\begin{aligned}
\widetilde{\mathbf{y}}_{\tau}^{*} & =\mathbf{h}+\boldsymbol{\alpha}_{s}+\widetilde{\mathbf{v}}_{s}^{*} \\
\mathbf{L}_{h} \mathbf{h} & =\mathbf{h}_{0}+\mathbf{u}^{h} \\
{\left[\begin{array}{c}
\widetilde{\mathbf{v}}_{s}^{*} \\
\mathbf{u}^{h}
\end{array}\right] } & \sim \mathcal{N}\left(\left[\begin{array}{l}
\mathbf{0} \\
\mathbf{0}
\end{array}\right] \quad\left[\begin{array}{cc}
\Sigma_{s} & 0 \\
0 & \boldsymbol{\Sigma}_{u^{h}}
\end{array}\right]\right) .
\end{aligned}
$$

Given the parametrization above, our MCMC sampler needs to be augmented to sample both $\mathbf{h}$ and the vector of component-density indicators, s. Formally, this entails sequentially sampling from the following full conditional posterior distributions: ${ }^{4}$

Step $1 f\left(\mathbf{s} \mid \widetilde{\mathbf{y}}_{\tau}^{*}, \mathbf{Z}\right)$

Step $2 f\left(\mathbf{h} \mid \widetilde{\mathbf{y}}_{\tau}^{*}, \mathbf{Z}_{-\mathbf{h}}, \mathbf{s}\right)$.

-Step 1

The auxiliary sampler of Omori et al. (2007) allows each element of $\mathbf{s}=\left(s_{1}, \cdots, s_{T}\right)$ to be drawn independently from a multinomial distribution parameterized by the full conditional posterior probabilities $\operatorname{Pr}\left(s_{t}=i \mid \widetilde{y}_{\tau, t}^{*}, Z_{t}\right)$ given by:

$$
\operatorname{Pr}\left(s_{t}=i \mid \widetilde{y}_{\tau, t}^{*}, z_{t}\right)=\frac{\psi\left(h_{t}+\alpha_{s=i}, \sigma_{s=i}^{2}\right) p_{s=i}}{\sum_{j=1}^{10} \psi\left(h_{t}+\alpha_{s=j}, \sigma_{s=j}^{2}\right) p_{s=j}} \quad \text { for } i=1, \cdots, 10
$$

where $\psi\left(h_{t}+\alpha_{s}, \sigma_{s}^{2}\right)$ denotes a Gaussian density evaluated at mean $h_{t}+\alpha_{s}$ and variance $\sigma_{s}^{2}$. Again, $\alpha_{s}$ and $\sigma_{s}^{2}$ values are given in Table 1 in Omori et al. (2007). $h_{t}$ denotes posterior draws obtained from (A19) as presented below.

Given $\operatorname{Pr}\left(s_{t}=i \mid \widetilde{y}_{\tau, t}^{*}, Z_{t}\right)$ posterior draws for $s_{t}$ can then be generated via the inverse

\footnotetext{
${ }^{4}$ Here, Steps 1 and 2 are consistent with the discussion in Del Negro and Primiceri (2015) that applies more broadly for models with stochastic volatility.
} 
transform method for $t=1, \cdots, T$ as follows: ${ }^{5}$

(a) Generate $e_{t} \sim$ Uniform $(0,1)$,

(b) Find the smallest $i \in\{1,2, \cdots, 10\}$ that satisfies $\sum_{j=1}^{i} \operatorname{Pr}\left(s_{t}=j \mid \widetilde{y}_{\tau, t}^{*}, Z_{t}\right) \geq e_{t}$,

(c) Return $\left(s_{t} \mid \widetilde{y}_{\tau, t}^{*}, Z_{t}\right)=i$.

-Step 2

$$
\mathbf{h} \mid \widetilde{\mathbf{y}}^{*}, \mathbf{Z}, \mathbf{s} \sim \mathcal{N}\left(\overline{\mathbf{d}}_{h}, \overline{\mathbf{D}}_{h}\right), \text { where }\left\{\begin{array}{l}
\overline{\mathbf{d}}_{h}=\overline{\mathbf{D}}_{h}\left(\boldsymbol{\Sigma}_{s}^{-1} \widetilde{\mathbf{y}}_{\alpha}^{*}+\mathbf{L}_{h}^{\prime} \boldsymbol{\Sigma}_{u^{h}}^{-1} \mathbf{h}_{0}\right) \\
\overline{\mathbf{D}}_{h}=\left(\boldsymbol{\Sigma}_{s}^{-1}+\mathbf{L}_{h}^{\prime} \boldsymbol{\Sigma}_{u^{h}}^{-1} \mathbf{L}_{h}\right)^{-1},
\end{array}\right.
$$

where $\widetilde{\mathbf{y}}_{\alpha}^{*}=\widetilde{\mathbf{y}}_{\tau}^{*}-\mathbf{h}-\boldsymbol{\alpha}_{s}$. Draws from the density above are obtained using the precision sampler of Chan and Jeliazkov (2009).

- Sampling $\gamma$

It's easy to see that the innovations in (A1) and (A2) can be factorized as follows:

$$
\begin{aligned}
& {\left[\begin{array}{c}
\boldsymbol{\zeta} \\
\mathbf{u}^{\tau}
\end{array}\right]=\left[\begin{array}{cc}
\mathbf{A}_{\zeta} & \mathbf{0} \\
\mathbf{0} & \mathbf{A}_{\tau}
\end{array}\right]\left[\begin{array}{c}
\boldsymbol{\zeta}_{*} \\
\mathbf{u}_{*}^{\tau}
\end{array}\right] \text { s.t. }} \\
& {\left[\begin{array}{c}
\boldsymbol{\zeta}_{*} \\
\mathbf{u}_{*}^{\tau}
\end{array}\right] \sim \mathcal{N}\left(\left[\begin{array}{l}
\mathbf{0} \\
\mathbf{0}
\end{array}\right],\left[\begin{array}{cc}
\boldsymbol{\Lambda}_{h \zeta} & \mathbf{0} \\
\mathbf{0} & \boldsymbol{\Lambda}_{h^{\tau}}
\end{array}\right]\right) .}
\end{aligned}
$$

Since $\left(\boldsymbol{\zeta}, \mathbf{u}^{\tau}\right)^{\prime}=\left(\boldsymbol{\zeta}^{G}, \boldsymbol{\zeta}^{S}, \mathbf{u}^{\tau^{G}}, \mathbf{u}^{\tau^{S}}\right)^{\prime}$ and using the fact that both $\mathbf{A}_{\zeta}$ and $\mathbf{A}_{\tau}$ have a

\footnotetext{
${ }^{5}$ See algorithm 3.2 in Kroese, Taimre and Botev (2013) for a more detailed discussion of the inverse transform method for discrete random variables.
} 
block-lower triangular structure - as shown in (A12) - then (A20) can be recast as:

$$
\left[\begin{array}{c}
\boldsymbol{\zeta}^{G} \\
\boldsymbol{\zeta}^{S} \\
\mathbf{u}^{\tau^{G}} \\
\mathbf{u}^{\tau^{S}}
\end{array}\right]=\left[\begin{array}{c}
\boldsymbol{\zeta}^{\zeta^{G}} \\
\mathbf{X}_{\gamma^{\zeta}} \boldsymbol{\zeta}^{G}+\boldsymbol{\zeta}_{*}^{S} \\
\mathbf{u}^{\tau^{G}} \\
\mathbf{X}_{\gamma^{\tau}} \mathbf{u}^{\tau^{G}}+\mathbf{u}_{*}^{\tau^{S}}
\end{array}\right]
$$

where $\mathbf{X}_{\gamma^{\ell}}=\operatorname{diag}\left(\gamma_{1}^{\ell}, \cdots, \gamma_{T}^{\ell}\right)$ for $\ell=\zeta$ and $\tau$.

Given the block-lower triangular structure of $\mathbf{A}_{\zeta}$ and $\mathbf{A}_{\tau}$ and the modular nature of MCMC algorithms, one can collect the draws associated with the goods sector in $\boldsymbol{\tau}$ and $\boldsymbol{\tau}_{0}-$ i.e. $\boldsymbol{\tau}^{G}$ and $\boldsymbol{\tau}_{0}^{G}$, respectively - to back out $\boldsymbol{\zeta}^{G}=\mathbf{y}^{G}-\boldsymbol{\tau}^{G}$ and $\mathbf{u}^{\tau^{G}}=\mathbf{H} \boldsymbol{\tau}^{G}-\boldsymbol{\tau}_{0}^{G}$. As a result, these two vectors of innovations can be treated as predetermined controls in the second and fourth equations in (A21). Similarly, we back out $\boldsymbol{\zeta}^{S}$ and $\mathbf{u}^{\tau^{S}}$ on the left-hand side of (A21) by setting $\boldsymbol{\zeta}^{S}=\mathbf{y}^{S}-\boldsymbol{\tau}^{S}$ and $\mathbf{u}^{\tau^{S}}=\mathbf{H} \boldsymbol{\tau}^{S}-\boldsymbol{\tau}_{0}^{S}$ and treat these vectors of innovations as regressands in a standard linear regression setting. Consequently, by a simple change of variables, we can use the second and fourth equations in (A21) to obtain the following state space representation for $\gamma^{6}$

$$
\begin{aligned}
\mathbf{e}^{S} & =\mathbf{X}_{e^{G}} \boldsymbol{\gamma}+\mathbf{e}_{*}^{S}, \\
\mathbf{L}_{\gamma} \boldsymbol{\gamma} & =\gamma_{0}+\mathbf{u}^{\gamma} \\
{\left[\begin{array}{c}
\mathbf{e}_{*}^{S} \\
\mathbf{u}^{\gamma}
\end{array}\right] } & \sim \mathcal{N}\left(\left[\begin{array}{l}
0 \\
0
\end{array}\right],\left[\begin{array}{cc}
\Sigma_{e_{*}^{S}} & 0 \\
0 & \boldsymbol{\Sigma}_{\gamma}
\end{array}\right]\right),
\end{aligned}
$$

\footnotetext{
${ }^{6}$ To be clear, we refer to the following change of variables: $\mathbf{X}_{\boldsymbol{\gamma}^{\zeta}} \boldsymbol{\zeta}^{G}$ and $\mathbf{X}_{\boldsymbol{\gamma}^{\tau}} \mathbf{u}^{\tau^{G}}$ can be equivalently expressed as diag $\left(\zeta_{1}^{G}, \cdots, \zeta_{T}^{G}\right) \gamma^{\zeta}$ and $\operatorname{diag}\left(u_{1}^{\tau^{G}}, \cdots, u_{T}^{\tau^{G}}\right) \boldsymbol{\gamma}^{\tau}$, respectively.
} 
where: $\mathbf{e}^{S}=\left[\begin{array}{c}\boldsymbol{\zeta}^{S} \\ \mathbf{u}^{\tau^{S}}\end{array}\right], \mathbf{e}_{*}^{S}=\left[\begin{array}{c}\boldsymbol{\zeta}_{*}^{S} \\ \mathbf{u}_{*}^{\tau^{S}}\end{array}\right]$,

$$
\begin{aligned}
& \boldsymbol{\Sigma}_{e_{*}^{S}}=\left[\begin{array}{cc}
\operatorname{diag}\left(\exp \left(h_{1}^{\zeta^{S}}\right), \cdots, \exp \left(h_{T}^{\zeta^{S}}\right)\right) & \mathbf{0} \\
\mathbf{0} & \operatorname{diag}\left(\exp \left(h_{1}^{\tau^{S}}\right), \cdots, \exp \left(h_{T}^{\tau^{S}}\right)\right)
\end{array}\right] \text { and } \\
& \mathbf{X}_{e^{G}}=\left[\begin{array}{cc}
\operatorname{diag}\left(\zeta_{1}^{G}, \cdots, \zeta_{T}^{G}\right) & \mathbf{0} \\
\mathbf{0} & \operatorname{diag}\left(u_{1}^{\tau^{G}}, \cdots, u_{T}^{\tau^{G}}\right)
\end{array}\right] .
\end{aligned}
$$

Thus, standard regression results can be applied to the system in (A22)-(A24) to obtain the following expressions for the full conditional posterior for $\gamma$ :

$$
\gamma \mid \mathbf{y}, \mathbf{Z}_{-\gamma} \sim \mathcal{N}\left(\overline{\mathbf{d}}_{\gamma}, \overline{\mathbf{D}}_{\gamma}\right), \text { where }\left\{\begin{array}{l}
\overline{\mathbf{d}}_{\gamma}=\overline{\mathbf{D}}_{\gamma}\left(\mathbf{X}_{e^{G}}^{\prime} \boldsymbol{\Sigma}_{e^{S}}^{-1} \mathbf{e}^{S}+\mathbf{L}_{\gamma}^{\prime} \boldsymbol{\Sigma}_{\gamma}^{-1} \gamma_{0}\right) \\
\overline{\mathbf{D}}_{\gamma}=\left(\mathbf{X}_{e^{G}}^{\prime} \boldsymbol{\Sigma}_{e^{S}}^{-1} \mathbf{X}_{e^{G}}+\mathbf{L}_{\gamma}^{\prime} \boldsymbol{\Sigma}_{\gamma}^{-1} \mathbf{L}_{\gamma}\right)^{-1}
\end{array}\right.
$$

Again, to obtain draws from the density above, we apply precision sampling methods.

\section{A1.2.2 Parameter Sampling}

- Sampling $\Omega_{h}$ and $\Omega_{\gamma}$

Recall that both $\Omega_{h}$ and $\Omega_{\gamma}$ are diagonal covariance matrices. Therefore, variance hyperparameters for the states $\mathbf{h}$ and $\boldsymbol{\gamma}$ can be sampled one by one from an inverse-gamma density. Formally, we have:

$$
\sigma_{\gamma^{\ell}}^{2} \mid \mathbf{y}, \mathbf{Z}_{-\sigma_{\gamma^{\ell}}^{2}} \sim \mathcal{I} \mathcal{G}\left(\bar{\nu}_{\gamma^{\ell}}, \bar{S}_{\gamma^{\ell}}\right), \text { where }\left\{\begin{array}{l}
\bar{\nu}_{\gamma^{\ell}}=\frac{T}{2}+\nu_{\gamma^{\ell}}, \\
\bar{S}_{\gamma^{\ell}}=\frac{\sum_{t=1}^{T}\left(u_{t}^{\gamma^{\ell}}\right)^{2}}{2}+S_{\gamma^{\ell}} \quad \text { for } \ell=\zeta \text { and } \tau,
\end{array}\right.
$$


and

$\sigma_{h^{\ell^{i}}}^{2} \mid \mathbf{y}, \mathbf{Z}_{-\sigma_{h^{\ell^{i}}}} \sim \mathcal{I} \mathcal{G}\left(\bar{\nu}_{h^{\ell^{i}}}, \bar{S}_{h^{\ell^{i}}}\right)$, where $\left\{\begin{array}{l}\bar{\nu}_{h^{\ell^{i}}}=\frac{T}{2}+\nu_{h^{\ell^{i}}}, \\ \bar{S}_{h^{\ell^{i}}}=\frac{\sum_{t=1}^{T}\left(u_{t}^{\ell^{\ell^{i}}}\right)^{2}}{2}+S_{h^{\ell^{i}}} \text { for } \ell=\zeta \text { and } \tau \text { and } i=G \text { and } S,\end{array}\right.$

where $u_{t}^{h^{\ell^{i}}}$ and $u_{t}^{\gamma^{\ell}}$ are the innovations in state equations (8) and (9), respectively, in Section 2.1.

\section{- Sampling $\mathrm{z}_{0}$}

Let $\mathbf{L}_{0}=I_{8} \otimes \iota_{0}$ where $\iota_{0}=(1,0, \cdots, 0)^{\prime}$. The state equations (A2), (A3) and (A4) can then be expressed as

$$
\mathbf{L} \mathbf{z}=\mathbf{L}_{0} \mathbf{z}_{0}+\mathbf{u}
$$

where $\mathbf{z}=\left[\begin{array}{c}\boldsymbol{\tau} \\ \mathbf{h} \\ \boldsymbol{\gamma}\end{array}\right], \mathbf{L}=\left[\begin{array}{ccc}\mathbf{L}_{\tau} & \mathbf{0} & \mathbf{0} \\ \mathbf{0} & \mathbf{L}_{h} & \mathbf{0} \\ \mathbf{0} & \mathbf{0} & \mathbf{L}_{\gamma}\end{array}\right]$ and $\mathbf{u}=\left[\begin{array}{c}\mathbf{u}^{\tau} \\ \mathbf{u}^{h} \\ \mathbf{u}^{\gamma}\end{array}\right] \sim \mathcal{N}\left(\mathbf{0}, \boldsymbol{\Sigma}_{u}\right)$, such that

$\Sigma_{u}=\left(\begin{array}{ccc}\Sigma_{\tau} & 0 & 0 \\ 0 & \Sigma_{h} & 0 \\ 0 & 0 & \Sigma_{\gamma}\end{array}\right)$

Combining (A28) with the Gaussian prior $\mathrm{z}_{0} \sim \mathcal{N}\left(\widehat{\mathrm{z}_{0}}, \boldsymbol{\Sigma}_{z_{0}}\right)$ yields

$$
\mathrm{z}_{0} \mid \mathbf{y}, \mathbf{Z}_{-\mathrm{z}_{0}} \sim \mathcal{N}\left(\overline{\mathbf{d}}_{\mathrm{z}_{0}}, \overline{\mathbf{D}}_{\mathrm{z}_{0}}\right), \text { where }\left\{\begin{array}{l}
\overline{\mathbf{d}}_{\mathrm{z}_{0}}=\overline{\mathbf{D}}_{\mathrm{z}_{0}}\left(\mathbf{L}_{0}^{\prime} \boldsymbol{\Sigma}_{u}^{-1} \mathbf{L} \mathbf{z}+\boldsymbol{\Sigma}_{z_{0}} \widehat{\mathrm{z}_{0}}\right) \\
\overline{\mathbf{D}}_{\mathrm{z}_{0}}=\left(\mathbf{L}_{0}^{\prime} \boldsymbol{\Sigma}_{u}^{-1} \mathbf{L}_{0}+\boldsymbol{\Sigma}_{z_{0}}^{-1}\right)^{-1}
\end{array}\right.
$$




\section{A2 Model Comparison}

Recall that our baseline model assumes that the transitory component of inflation in each sector follows a normally distributed serially uncorrelated process. To evaluate the ability of such a specification to fit inflation data, we conduct a model comparison exercise where we allow for persistence in the transitory component of (sector-specific) inflation. We propose two alternatives to model sectoral inflation gap, $c_{t}^{i}$, for $i=G$ and $S$, namely - and similar to previous studies (e.g., see Chan, Koop and Potter, 2013, 2016) - gaps are modeled as an $\mathrm{AR}(1)$ and as a time-varying parameter (TVP) AR process, i.e.:

- Two-Sector UC-SV-AR

$$
c_{t}^{i}=\phi^{i} c_{t-1}+\zeta_{t}^{i}, \quad \text { for } i=G \text { and } S
$$

\section{- Two-Sector UC-SV-TVP-AR}

$$
\begin{aligned}
c_{t}^{i} & =\phi_{t}^{i} c_{t-1}+\zeta_{t}^{i}, \\
\phi_{t}^{i} & =\phi_{t-1}^{i}+u_{t}^{\phi^{i}}, \quad u_{t}^{\phi^{i}} \sim \mathcal{N}\left(0, \sigma_{\phi^{i}}^{2}\right) \text { for } i=G \text { and } S .
\end{aligned}
$$

Table (A1) reports estimates of the log marginal likelihood, which is the metric for model comparison we adopt. To compute the log marginal likelihood, we follow Geweke and Amisano (2011) and carry out a recursive one-step-ahead forecasting exercise. ${ }^{7}$ Applying the usual recommendations for interpreting the Bayes factor - i.e. the ratio between the marginal likelihood from two competing models - as in, e.g., Raftery (1995), one can see that there is very strong evidence in favor of our assumption to model sector-specific inflation gaps a serially uncorrelated processes, thus reinforcing our choice to follow Stock and Watson (2007) and Stock and Watson (2016) on how to model the transitory component of inflation.

\footnotetext{
${ }^{7}$ In this forecasting procedure, we start from the fifth observation in our sample for each sectoral inflation to reduce the sensitivity to priors.
} 
Table A1: Model comparison results based on predictive simulations

\begin{tabular}{lc}
\hline \hline Identifier & Log Marginal Likelihood Estimates \\
\hline Two-Sector UC-SV & $\mathbf{- 2 2 7 9 . 4}$ \\
Two-Sector UC-SV-AR & -2593.2 \\
Two-Sector UC-SV-TVP-AR & -2876.8 \\
\hline \hline
\end{tabular}

\section{A2.1 Extensions to the Baseline MCMC Algorithm}

To accommodate an AR transitory component, we slightly modify the stacked representation in (A1). In particular, for the Two-Sector UC-AR and Two-Sector UC-TVP-AR models we express the measurement equation as

$$
\mathbf{y}=\boldsymbol{\tau}+\mathbf{c},
$$

where

$$
\underset{(2 T \times 1)}{\mathbf{c}}=\left[\begin{array}{c}
\left(c_{1}^{G}, \cdots, c_{T}^{G}\right)^{\prime} \\
\left(c_{1}^{S}, \cdots, c_{T}^{S}\right)^{\prime}
\end{array}\right] .
$$

- Two-Sector UC-SV-AR

The only additional parameters in the case of the Two-Sector UC-AR model are the AR coefficients for the sector-specific gaps. The (stacked) representation of the state equation for $\mathbf{c}$ is given by

$$
\mathbf{c}=\mathbf{X}_{c} \phi+\zeta
$$


where

$$
\underset{(2 T \times 2)(2 \times 1)}{\mathbf{X}_{c} \boldsymbol{\phi}}=\underbrace{\left[\begin{array}{cc}
0 & 0 \\
c_{1}^{G} & 0 \\
\vdots & \vdots \\
c_{T-1}^{G} & 0 \\
0 & 0 \\
0 & c_{1}^{S} \\
\vdots & \vdots \\
0 & c_{T-1}^{S}
\end{array}\right]}_{\mathbf{X}_{c}} \underbrace{\left[\begin{array}{c}
\phi^{G} \\
\phi^{S}
\end{array}\right]}_{\phi} .
$$

We employ a Gaussian prior for $\phi$. Specifically, we set $\phi \sim \mathcal{N}\left(\boldsymbol{\mu}_{\phi}, \boldsymbol{\Sigma}_{\phi}\right)$ such that $\boldsymbol{\mu}_{\phi}=$ $(0.5,0.5)^{\prime}$ and $\Sigma_{\phi}=10^{-3} \times I_{2}$. Combining such prior with (A34) yields the following full conditional posterior for $\phi$ :

$$
\phi \mid \mathbf{y}, \mathbf{Z}_{-\phi} \sim \mathcal{N}\left(\overline{\mathbf{d}}_{\phi}, \overline{\mathbf{D}}_{\phi}\right), \text { where }\left\{\begin{array}{l}
\overline{\mathbf{d}}_{\phi}=\overline{\mathbf{D}}_{\phi}\left(\mathbf{X}_{c}^{\prime} \boldsymbol{\Sigma}_{\zeta}^{-1} \mathbf{c}+\boldsymbol{\Sigma}_{\phi}^{-1} \boldsymbol{\mu}_{\phi}\right) \\
\overline{\mathbf{D}}_{\tau}=\left(\mathbf{X}_{c}^{\prime} \boldsymbol{\Sigma}_{\zeta}^{-1} \mathbf{X}_{c}+\boldsymbol{\Sigma}_{\phi}^{-1}\right)^{-1}
\end{array}\right.
$$

- Two-Sector UC-SV-TVP-AR

When assigning a law of motion to the AR coefficients, the model is augmented by an additional state equation plus two sets of parameters, namely the initial conditions for the new state variables and variances for the innovations associated with such states. Formally, we have:

$$
\begin{aligned}
\mathbf{c} & =\mathbf{X}_{c} \boldsymbol{\phi}+\boldsymbol{\zeta} \\
\mathbf{L}_{\phi} \boldsymbol{\phi} & =\phi_{0}+\mathbf{u}^{\phi}, \quad \mathbf{u}^{\phi} \sim \mathcal{N}\left(\mathbf{0}, \boldsymbol{\Sigma}_{\phi}\right),
\end{aligned}
$$


where

$$
\begin{aligned}
& \underset{(2 T \times 2 T)}{\mathbf{X}_{c}}=\left[\begin{array}{cc}
\operatorname{diag}\left(0, c_{1}^{G}, \cdots, c_{T-1}^{G}\right) & \mathbf{0} \\
\mathbf{0} & \operatorname{diag}\left(0, c_{1}^{S}, \cdots, c_{T-1}^{S}\right)
\end{array}\right], \quad \underset{(2 T \times 1)}{\boldsymbol{\phi}}=\left[\begin{array}{c}
\left(\phi_{1}^{G}, \cdots, \phi_{T}^{G}\right)^{\prime} \\
\left(\phi_{1}^{S}, \cdots, \phi_{T}^{S}\right)^{\prime}
\end{array}\right], \\
& \underset{(2 T \times 2 T)}{\boldsymbol{\Sigma}_{\phi}}=\left[\begin{array}{cc}
\sigma_{\phi^{G}}^{2} I_{T} & \mathbf{0} \\
\mathbf{0} & \sigma_{\phi^{S}}^{2} I_{T}
\end{array}\right], \underset{(2 T \times 2 T)}{\underset{\mathbf{L}_{\phi}}{\mathbf{0}}}=\left[\begin{array}{cc}
\mathbf{H} & \mathbf{0} \\
\mathbf{0}
\end{array}\right], \text { such that: } \underset{(T \times T)}{\mathbf{H}}=\left[\begin{array}{ccccc}
1 & 0 & 0 & \cdots & 0 \\
-1 & 1 & 0 & \cdots & 0 \\
0 & -1 & 1 & & \vdots \\
\vdots & \vdots & \ddots & \ddots & \\
0 & 0 & \cdots & -1 & 1
\end{array}\right] .
\end{aligned}
$$

Initialization conditions for $\phi$ are collected as

$$
\underset{(2 T \times 1)}{\phi_{0}}=\left[\begin{array}{c}
\phi_{0}^{G} \\
\phi_{0}^{S}
\end{array}\right] \otimes\left[\begin{array}{c}
1 \\
0 \\
\vdots \\
0
\end{array}\right]
$$

Combining (A36) and (A37) yields the following full conditional posterior for $\phi$ :

$$
\phi \mid \mathbf{y}, \mathbf{Z}_{-\phi} \sim \mathcal{N}\left(\overline{\mathbf{d}}_{\phi}, \overline{\mathbf{D}}_{\phi}\right), \text { where }\left\{\begin{array}{l}
\overline{\mathbf{d}}_{\phi}=\overline{\mathbf{D}}_{\phi}\left(\mathbf{X}_{c}^{\prime} \boldsymbol{\Sigma}_{\zeta}^{-1} \mathbf{c}+\mathbf{L}_{\phi}^{\prime} \boldsymbol{\Sigma}_{\phi}^{-1} \phi_{0}\right) \\
\overline{\mathbf{D}}_{\tau}=\left(\mathbf{X}_{c}^{\prime} \boldsymbol{\Sigma}_{\zeta}^{-1} \mathbf{X}_{c}+\mathbf{L}_{\phi}^{\prime} \boldsymbol{\Sigma}_{\phi}^{-1} \mathbf{L}_{\phi}\right)^{-1}
\end{array}\right.
$$

Drawing from the density above is carried out using the precision sampling methods discussed in Section A1.2.1. Finally, sampling $\left\{\sigma_{\phi^{G}}^{2}, \sigma_{\phi^{S}}^{2}\right\}$ and $\left\{\phi_{0}^{G}, \phi_{0}^{S}\right\}$ follows the same steps as in (A26) and (A29), respectively. 


\section{A3 Additional Results from Two-Sector UC-SV}

\section{A3.1 Credible Sets of Trend Inflation Estimate}

Figure A1 presents the posterior median of our sector-specific and aggregate trend inflation estimate together with the bounds of the $67 \%$ credible set. All results are in terms of annualized quarter-on-quarter inflation, which corresponds with the units of the data which enter the estimated model. In general, the estimates appear to be fairly precisely estimated.

\section{A3.2 Comparison Relative to Stock and Watson (2007) UUC-SV}

We compare our model to the UUC-SV model by Stock and Watson (2007). For completeness, the Stock and Watson (2007) UUC-SV model is described as follows:

$$
\begin{aligned}
\pi_{t} & =\tau_{t}+\zeta_{t} \\
\tau_{t} & =\tau_{t-1}+u_{t}^{\tau} \\
h_{t} & =h_{t-1}+u_{t}^{h^{j}}, j \in\{\tau, \zeta\} \\
u_{t}^{\tau} & \sim N\left(0, \exp \left(h_{t}^{\tau}\right)\right), \zeta_{t} \sim N\left(0, \exp \left(h_{t}^{\zeta}\right)\right),
\end{aligned}
$$

where aggregate trend inflation, $\pi_{t}$, is decomposed driftless random walk trend, $\tau_{t}$ and a transitory noise component, $\zeta_{t}$, where both components have laws of motions that allow for stochastic volatility in their respective innovations. Note that there is zero correlation between the innovations to trend and transitory noise, as per Stock and Watson (2007) and Stock and Watson (2016). As should be clear, the key difference between our two-sector UC-SV model and the Stock and Watson (2007) UUC-SV model is that we split aggregate inflation into the services and goods sectors. All other features, such as allowing for stochastic volatility in the variance of the components and the random walk without drift specification for the random walk component, are identical to our model.

The top panel of Figure A2 compares the trend inflation estimate from the two-sector model relative to the UUC-SV. The bottom two panels of Figure A2 compares the estimates 
Figure A1: Posterior Distribution of Sector-Specific and Aggregate Trend Inflation
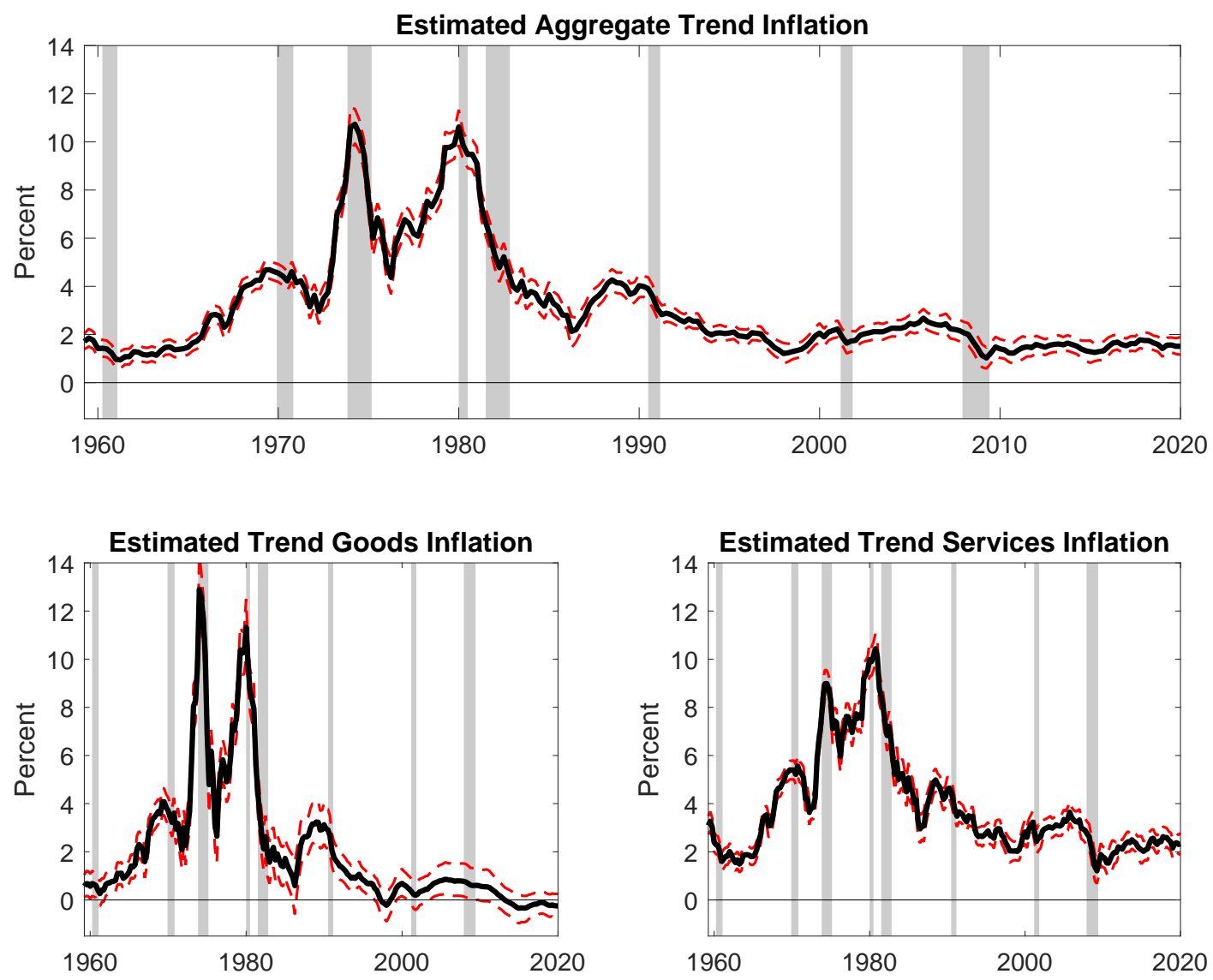

Notes: Shaded bars are NBER dated recessions. Trend inflation estimates are obtained as the posterior median estimates from our model. Units in terms of annualized quarter-on-quarter inflation. Dotted lines represent $67 \%$ credible sets.

of aggregate trend inflation volatility from both the UUC-SV model and the two-sector UC-SV model. All results are in terms of annualized quarter-on-quarter inflation, which corresponds with the units of the data which enter the estimated model.

We find that the level of the trend inflation estimate from both models largely mimics one another. In fact, before the Great Recession, the trend inflation estimates from the twosector model and the UUC-SV model are indistinguishable from one another. There appears to be some divergence during and just after the Great Recession where the trend inflation estimate from our two-sector UC-SV model was slightly lower than the UUC-SV, though by around 2014, the trend inflation estimates from both models appear to have coincided 
Figure A2: Comparison of Two-Sector UC-SV and UUC-SV

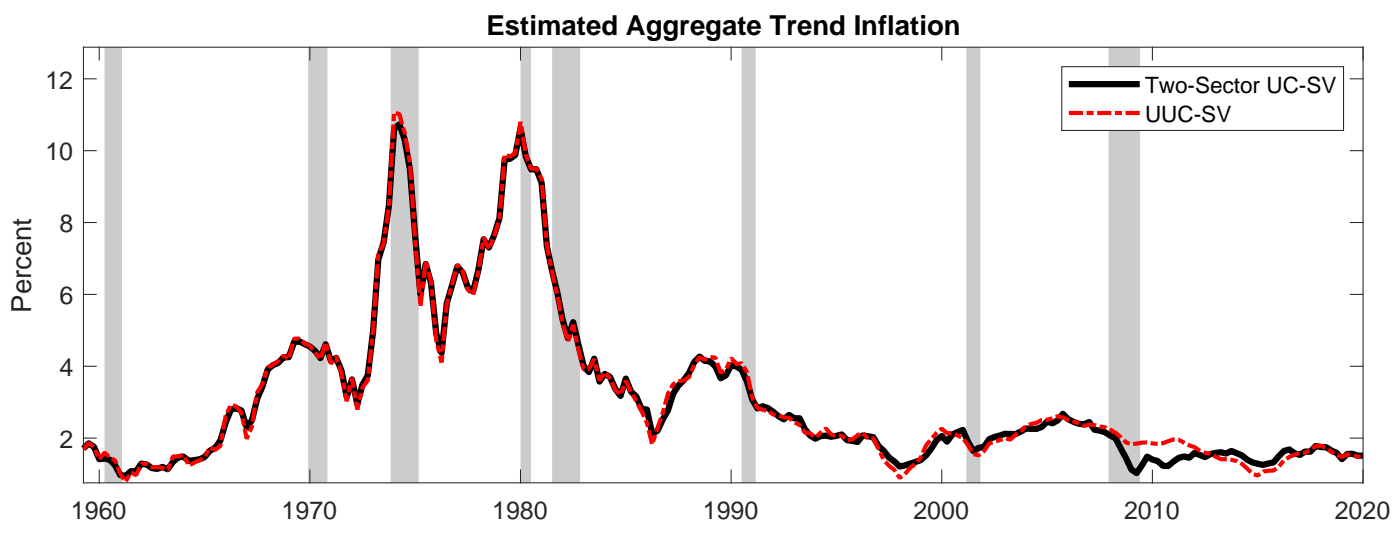

Estimated Standard Deviation of Innovations to Aggregate Trend Inflation
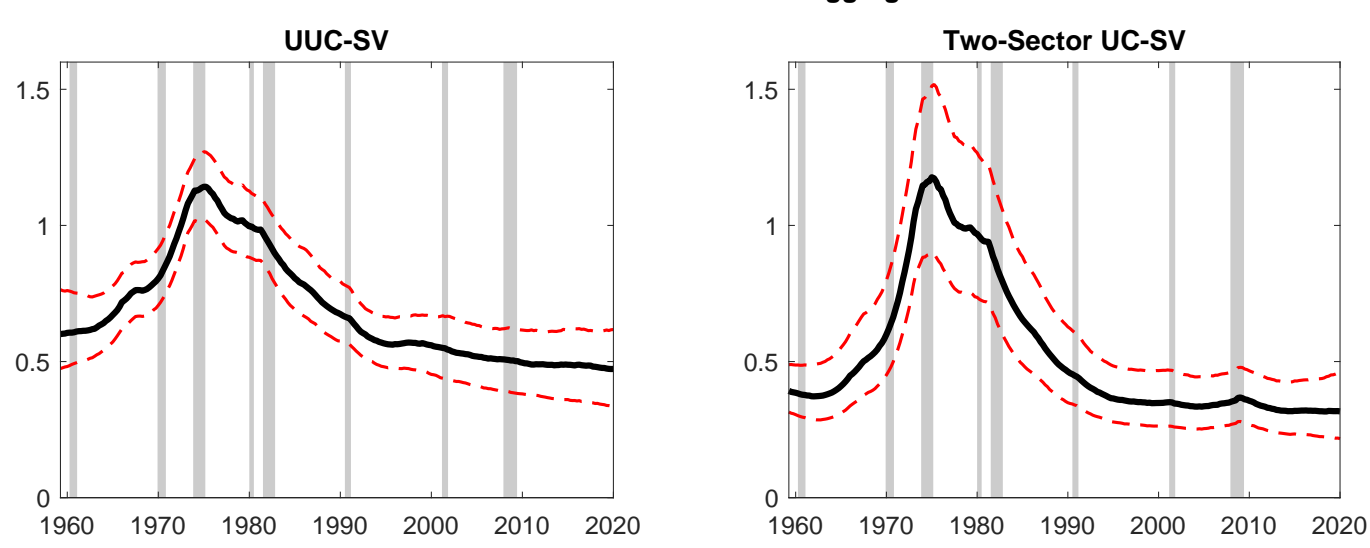

Notes: Shaded bars are NBER dated recessions. All units are in terms of annualized quarteron-quarter inflation. Top Panel: Posterior median trend inflation estimate from two-sector $U C-S V$ and $U U C-S V$. Bottom panels: Estimated standard deviation of innovations to trend inflation for two-sector $U C-S V$ and $U U C-S V$. Posterior median estimate with $67 \%$ credible sets.

once again. On the estimated standard deviation of the innovations to trend inflation, the bottom two panels of Figure A2 show that the variance of aggregate trend inflation from the two-sector UC-SV has essentially the same pattern as the UUC-SV. While it is qualitatively similar, we do note that the estimate of the variance of aggregate trend inflation from the two-sector UC-SV is slightly larger during the Great Inflation, and about half that of the UUC-SV around the end of the sample.

We also conducted a pseudo out-of-sample forecasting exercise for 1 to 12 quarter ahead 
Figure A3: Forecasting Performance
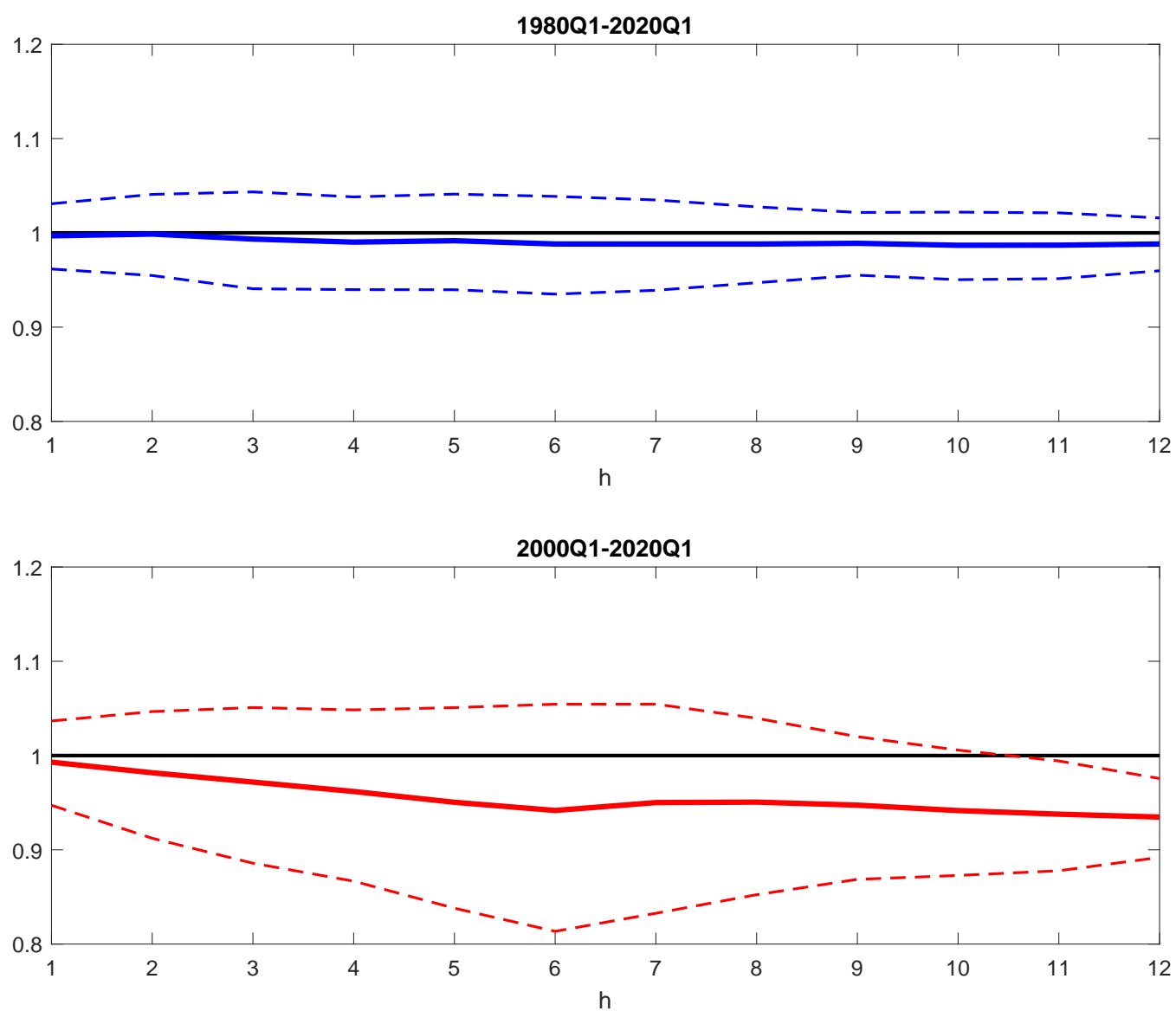

Notes: Results of a pseudo out-of-sample forecasting exercise of inflation for two sample periods: 1980Q1-2019Q2 and 2000Q1-2019Q2. The results are reported in terms of the relative root mean squared error (RRMSE) of the inflation forecast of the two-sector $U C-S V$ model relative to the UUC-SV model. Values below 1 indicate superior forecasting performance of the two-sector UC-SV model. The solid line reports the RRMSE. The dotted line indicates the 90\% confidence interval of the RRMSE by inverting the Diebold and Mariano (1995) test statistic. The $x$-axis represents the forecast horizon in terms of the $h$ quarter ahead out of sample RRMSE of the forecasts.

PCE inflation. ${ }^{8}$ Recall that since the transitory component does not feature dynamics, the h-step ahead forecast of inflation is always the current estimate of trend inflation or

\footnotetext{
${ }^{8}$ To be precise, let $p_{t}$ be the natural $\log$ of the PCE index. We define annualized quarter-on-quarter inflation, $\pi_{t}=400 \times\left[p_{t}-p_{t-1}\right]$. When we compute an $h$-step-ahead forecast, we are forecasting $\frac{1}{h} \sum_{j=1}^{h} \pi_{t+j}$ with the model estimated up to time $t$. This is equivalent to forecasting $\frac{1}{h} 400 \times\left[p_{t+h}-p_{t}\right]$, which is the change in the price level from $t$ to $t+h$, with a scaling factor to account for the annualization and the horizon.
} 


$$
\mathbb{E}_{t} \pi_{t+h}=\tau_{t} \forall h>0
$$

Figure A3 presents the results of the out-of-sample forecasting exercise. We report the out-of-sample relative root mean squared error (RRMSE) of the two-sector UC-SV relative to the UUC-SV. Therefore, values below 1 denote superior forecasting performance of the two-sector model and vice versa. The solid line reports the RRMSE and the dotted lines are the bounds of the $90 \%$ confidence interval of the RRMSE which we construct based on the Diebold and Mariano (1995) test for forecast accuracy. In general, the forecasting performance of both models is comparable. Realistically, given the similarities of the aggregate trend inflation estimates from both the two-sector and UUC-SV model, one should not expect sizeable outperformance of either model. For the forecast evaluation period of 1980Q1 to 2020Q1, the inflation forecasts of both models are statistically indistinguishable from one another. Nonetheless, we do detect slightly superior forecasting performance of the two-sector UC-SV model when we shift the forecast evaluation period to 2000Q1-2020Q1. The superior forecasts of the two-sector UC-SV model in the period 2000Q1-2019Q2 are statistically significant for the 10-12 quarter ahead forecast based on the Diebold and Mariano (1995) test, with gains of up to about $7 \%$ at particular horizons. We note from Figure A2 that the trend inflation estimates from both models is slightly different between 2008-2013, and we view it as a positive that our slightly different trend inflation did not compromise, and indeed may have even improved, the inflation forecast relative to the UUC-SV.

Overall, we find that the implications for aggregate trend inflation which we obtain from our two-sector UC-SV model are comparable to those one obtains from the UUC-SV model by Stock and Watson (2007). We essentially obtain similar estimates for aggregate trend inflation and the general pattern of the rise, then fall of the permanent component of aggregate trend inflation. We also obtain comparable forecasts to the UUC-SV. 


\section{A4 Details on Implementing Identification of the Pure Inflation Component}

We just restate the main equations for ease of reference. The sector-specific trend inflation can be represented with two components: pure trend inflation and sectoral permanent changes in the relative prices as follows:

$$
\begin{aligned}
\tau_{t}^{G} & =f_{t}+\tau_{t}^{G *}, \\
\tau_{t}^{S} & =f_{t}+\tau_{t}^{S *},
\end{aligned}
$$

where $f_{t}$ denotes pure trend inflation that is common to both sectors, and $\tau_{t}^{G *}$ and $\tau_{t}^{S *}$ captures the relative trend inflation for goods and services, respectively. These three state variables evolve as follows:

$$
\begin{aligned}
f_{t} & =f_{t-1}+\nu_{t}^{f}, \\
\tau_{t}^{G *} & =\tau_{t-1}^{G *}+\nu_{t}^{\tau^{G *}}, \\
\tau_{t}^{S *} & =\tau_{t-1}^{S *}+\nu_{t}^{\tau^{S *}} .
\end{aligned}
$$

To identify each latent component, we impose some restrictions as in Reis and Watson (2010). ${ }^{9}$ Specifically, albeit accounting for three state variables $\left(f_{t}, \tau_{t}^{G *}\right.$ and $\left.\tau_{t}^{S *}\right)$, the model in (A39)-(A43) only requires two exogenous shocks, namely a pure trend inflation and a relative trend inflation shock. This implies that $\nu_{t}^{\tau^{G *}}$ and $\nu_{t}^{\tau^{S *}}$ should be perfectly negatively correlated in order to characterize a relative price shock as a driver that moves the two sectoral trends in the exact opposite directions at all points in time. Moreover, since both pure inflation and relative price shocks have permanent effects on the sectoral trend inflation rates $\left(\tau_{t}^{G}\right.$ and $\left.\tau_{t}^{S}\right)$, to distinguish one from the other, $f_{t}$ is assumed to be independent from

9 Reis and Watson (2010) consider a dynamic factor model to extract $k$ factors. The $k$ factors are then decomposed into one common component (pure inflation) and $k-1$ relative price components. The imposed restrictions in our decomposition for the two-sector model would be similar to those for their model with $k=2$ sectors. Thus, our model requires one pure trend inflation component and one relative price component. Also, they find that, among $k$ factors, two factors have unit roots: one is associated with pure inflation and the other is associated with one of the relative price components. Their empirical finding of the permanent components is exactly consistent with our trend inflation decomposition framework. 
$\tau_{t}^{G *}$ and $\tau_{t}^{S *}$. These imposed restrictions result in a variance-covariance matrix given by:

$$
\left[\begin{array}{c}
\nu_{t}^{f} \\
\nu_{t}^{\tau^{G *}} \\
\nu_{t}^{\tau^{S *}}
\end{array}\right] \sim \mathcal{N}\left(\left[\begin{array}{l}
0 \\
0 \\
0
\end{array}\right],\left[\begin{array}{ccc}
\sigma_{f, t}^{2} & 0 & 0 \\
0 & \sigma_{\tau^{G *}, t}^{2} & -\sigma_{\tau^{G *}, t} \sigma_{\tau^{S *}, t} \\
0 & -\sigma_{\tau^{G *}, t} \sigma_{\tau^{S *}, t} & \sigma_{\tau^{S *}, t}^{2}
\end{array}\right]\right) .
$$

Table A2: Second Moment Restrictions: perfectly negatively correlated Sector-Specific Shocks

\begin{tabular}{lcc}
\hline \hline Second Moments & UC Model & Pure Inflation Decomposition \\
\hline$\sigma_{\tau^{G}, t}^{2}$ & $\gamma_{t}^{2} \exp \left(h_{g, t}\right)$ & $\sigma_{f, t}^{2}+\sigma_{\tau^{G *}, t}^{2}$ \\
$\sigma_{\tau^{S}, t}^{2}$ & $\gamma_{t}^{2} \exp \left(h_{g, t}\right)+\exp \left(h_{s, t}\right)$ & $\sigma_{f, t}^{2}+\sigma_{\tau^{S *}, t}^{2}$ \\
$\sigma_{\tau, t}$ & $\gamma_{t} \exp \left(h_{g, t}\right)$ & $\sigma_{f, t}^{2}-\sigma_{\tau^{G *}, t} \sigma_{\tau^{S *}, t}$ \\
\hline \hline
\end{tabular}

Next, we extract $f_{t}, \tau_{t}^{G *}$ and $\tau_{t}^{S *}$ by applying Kalman smoothing recursions to the state space model given by Equations (A39)-(A43). Note that the estimates (posterior medians) of $\tau_{t}^{G}, \tau_{t}^{G}, \sigma_{\tau^{G}, t}^{2}, \sigma_{\tau^{S}, t}^{2}$, and $\sigma_{\tau, t}$ are readily available from our Two-Sector UC-SV model. In particular, the three (time-varying) second moment parameters in the covariance matrix in (A44) are backed out via a matching moment approach using the implied relationship between $\sigma_{f, t}^{2}, \sigma_{\tau^{G *}, t}^{2}, \sigma_{\tau^{S *}, t}^{2}$ and $\sigma_{\tau^{G}, t}^{2}, \sigma_{\tau^{S}, t}^{2}, \sigma_{\tau, t}$ reported in Table A2. 


\section{A5 Excluding Energy}

\section{A5.1 Constructing the Goods Ex-Energy and Services Ex-Energy Series}

To construct the goods ex-energy and services ex-energy series, we use data from BEA Tables 2.3.4 (Price Indexes for Personal Consumption Expenditures by Major Type of Product) and 2.3.5 (Personal Consumption Expenditures by Major Type of Product), which provides a breakdown of components of the goods and/or services deflator that is made up of energy goods and energy services, respectively. We use both price indexes and expenditure weights

Figure A4: Goods and Services Excluding Energy Inflation
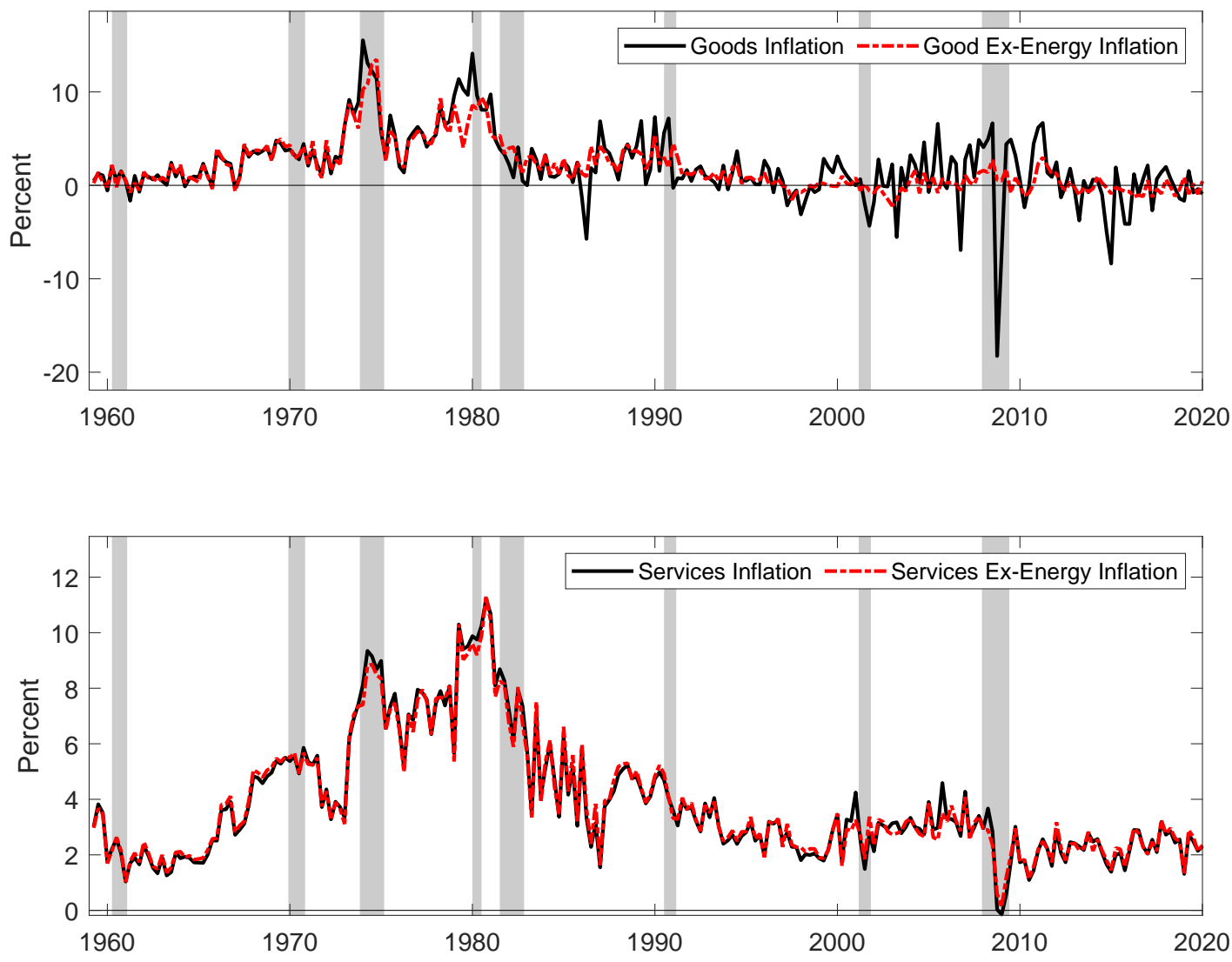

Notes: Inflation in terms of annualized quarter-on-quarter inflation. 
for Goods; Services; Gasoline and other energy goods, which are energy components for goods; and Energy goods and services, which are energy components for both goods and services because energy components for services only are not available in the tables. We extract the energy components for services by taking out the energy goods component from the component that contains both energy goods and services. We then construct chainweighted measures of goods and services inflation rates excluding energy components based on Whelan's (2002) suggestion of a Tornqvist formula, which is close to the ideal Fisher formula.

Figure A4 plots the constructed goods and services excluding energy inflation series which we constructed. We also plot goods and services inflation as a point of reference. As the energy component of inflation is largely in goods, and less so in services, it is thus not surprising that the goods excluding energy inflation series does deviate from goods inflation. In particular, goods inflation does inherit much of the volatility in energy inflation, which forms part of our motivation of estimating a model by excluding the energy component. On the other hand, services excluding energy inflation tracks services inflation very closely, a reflection of the small energy share in services inflation.

\section{A5.2 Other Results with Ex-Energy Data}

Figure A5 presents the estimated sector-specific and trend inflation estimates when we exclude energy components. For goods inflation, the estimates differ most during the late 1970s, and differ slightly during the early to mid-2000s. The difference during the 1979/80 oil shock is sufficiently large for the estimated trend goods inflation that the aggregate trend inflation estimates are sufficiently different during that period. Otherwise, the sector-specific and aggregate trend inflation estimates are reasonably similar when one includes or excludes energy components.

Figure A6 compares the estimated standard deviation of the transitory component when we estimate the model with and without energy. As we can see, much of the transitory noise component of goods inflation reflects fluctuations in energy prices. We state nonetheless that while excluding energy, while it has a meaningful impact on the estimates of the noise component of goods inflation, our conclusions on trend inflation in the main text are insensitive 
Figure A5: Estimated Trend Inflation
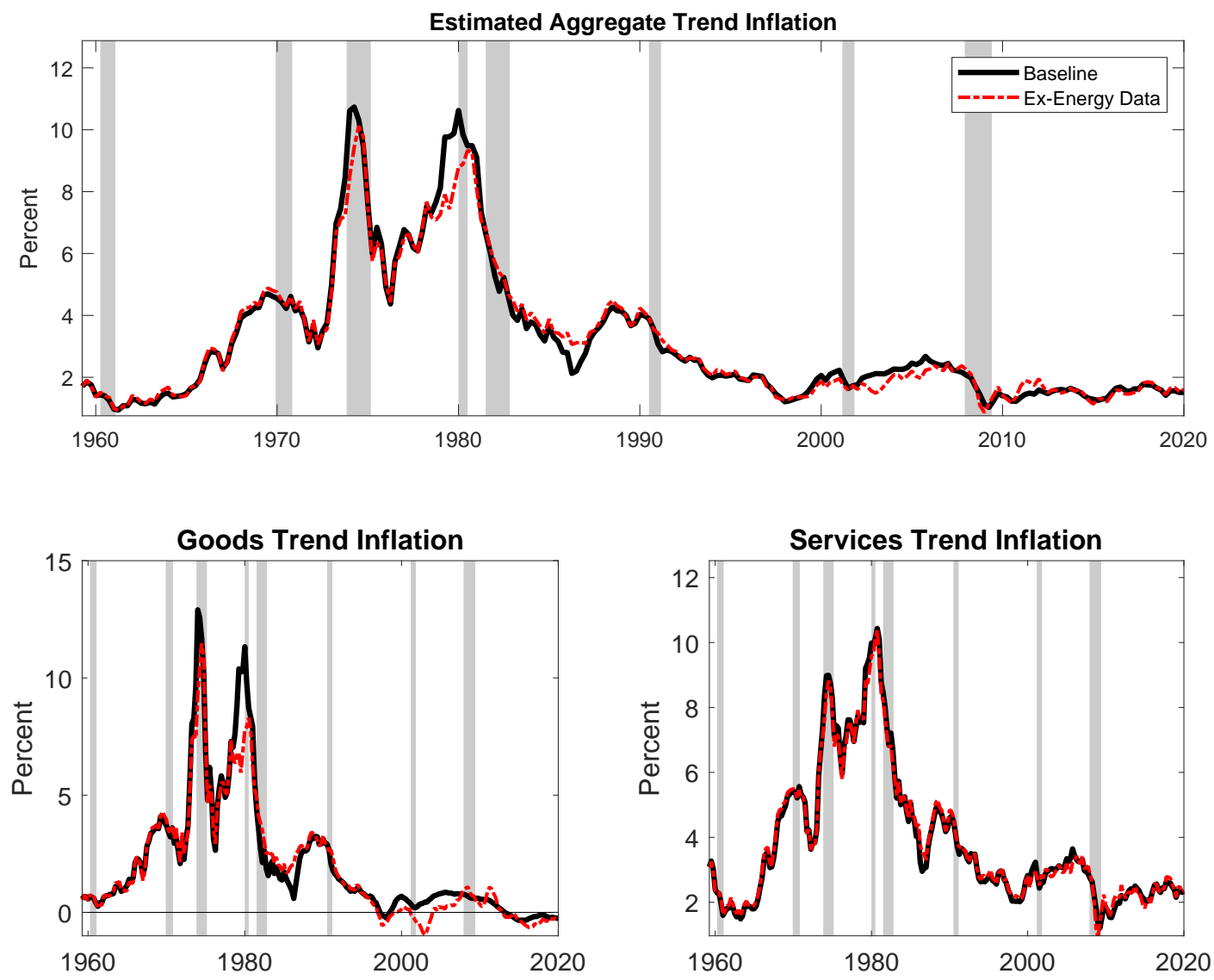

Notes: Shaded bars are NBER dated recessions. All units are in terms of annualized quarteron-quarter inflation. Baseline refers to the model estimated in the main text. The dotted lines are estimates using data that exclude energy components in both goods and services.

to the inclusion or exclusion of energy prices.

As energy prices may interfere with the identification of the pure inflation component, Figure A7 presents the decomposition when we use the ex-energy series for our estimation. This is analogous to Figure 9 in the main text. Our results remain almost identical to the results in the main text, which suggests our conclusions about the role of pure inflation is robust to the inclusion or non-inclusion of energy components in the goods and services inflation. 
Figure A6: Estimated Trend Inflation

(a) Baseline
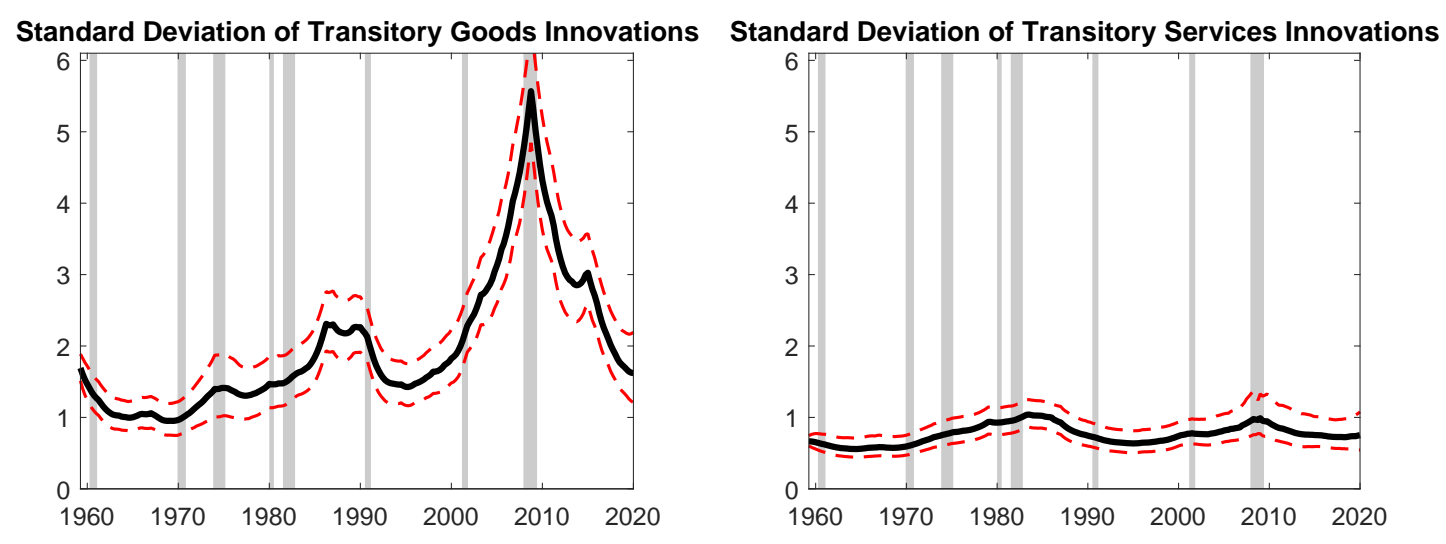

(b) Ex-Energy Data
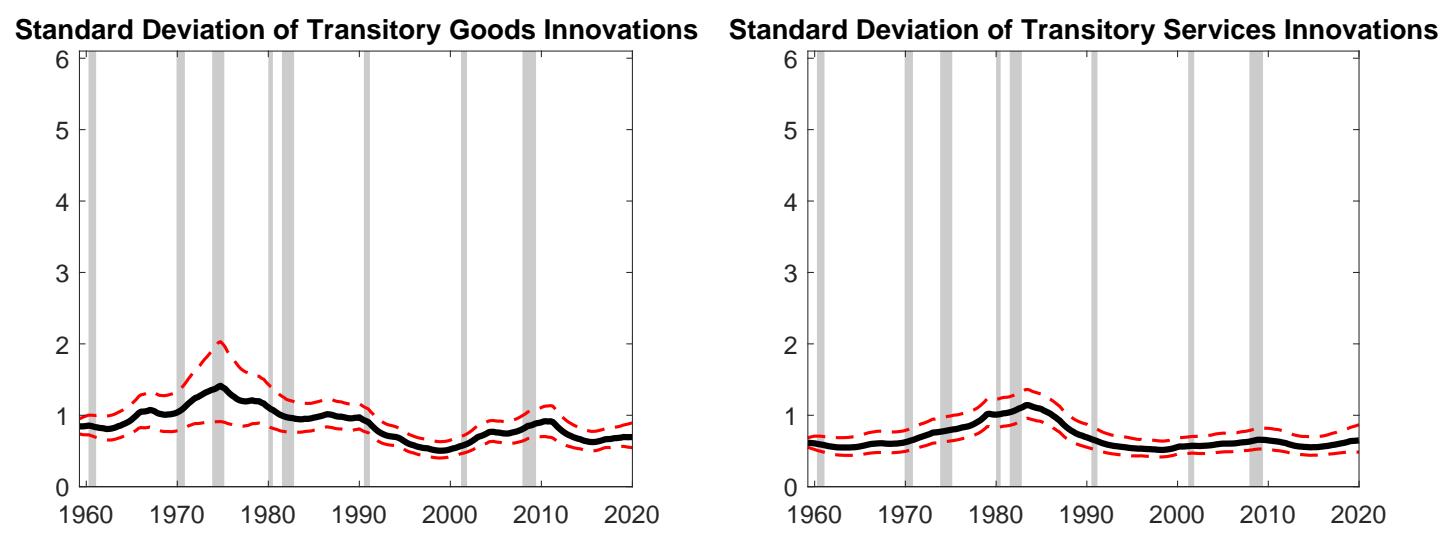

Notes: Shaded bars are NBER dated recessions. All units are in terms of annualized quarteron-quarter inflation. Baseline refers to the model estimated in the main text. The dotted lines are estimated using data that exclude energy components in both goods and services. Posterior median estimate with associated $67 \%$ posterior credible interval.

\section{A6 Australia and Canada Data}

\section{A6.1 Construction of Goods and Services Deflators for Australia and Canada}

Australia does not provide a goods and services breakdown. We construct the goods and services deflators by once again using the Household Final Consumption Expenditure in the 
Figure A7: Decomposition into Pure Inflation and Relative Price Components
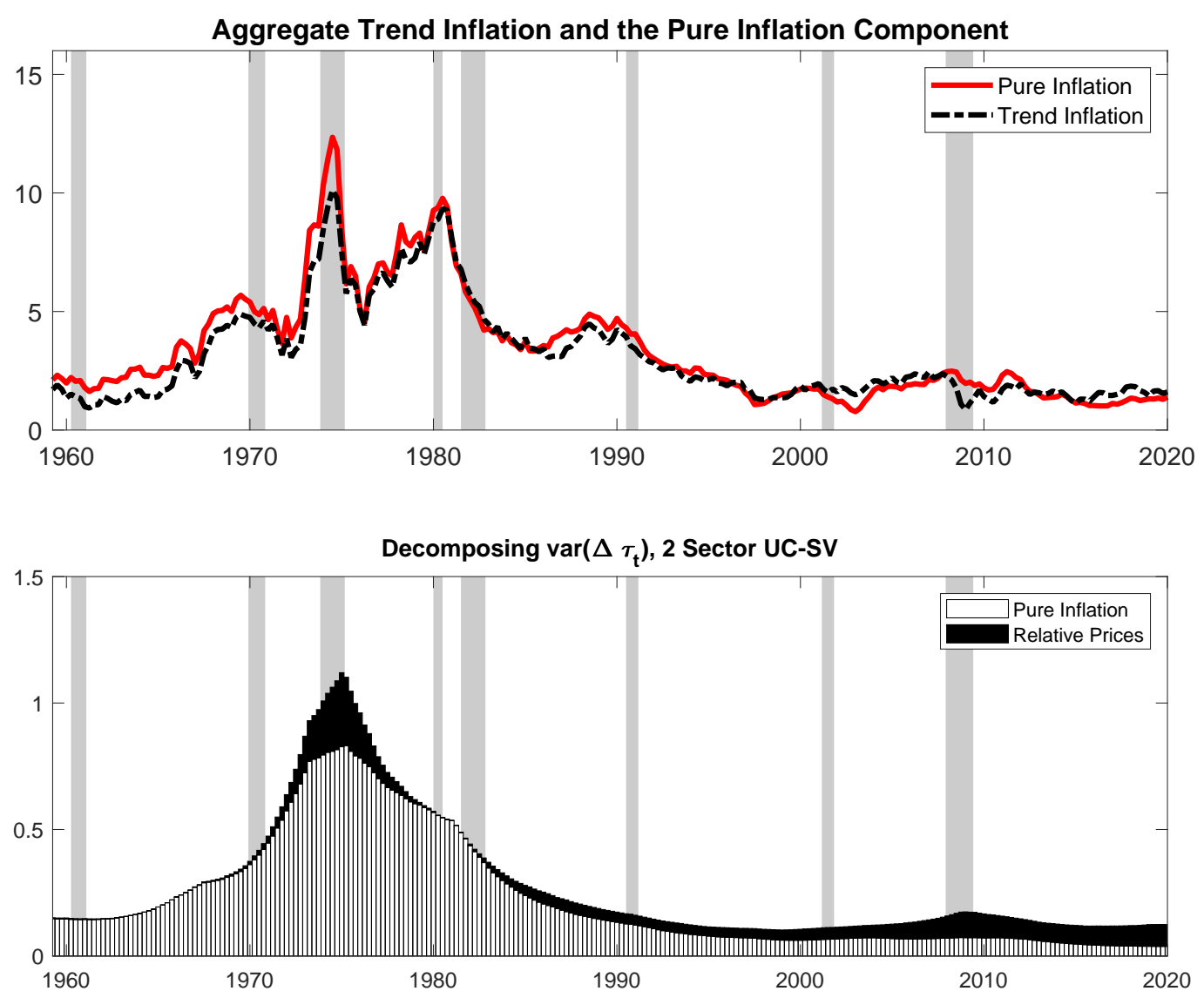

Notes: Shaded bars are NBER dated recessions. Trend inflation is in units of annualized quarter-on-quarter inflation.

quarterly national accounts, provided by the Australian Bureau of Statistics (ABS). ${ }^{10}$ The breakdown of consumption expenditure for goods and services in Australia is not reported. Instead, categories for consumption expenditure is reported. We classified the following as services: Rent and other dwelling services, Electricity, gas and other fuel, Furnishings and household equipment, Health Operation of vehicles, Transport services, Communications, Recreation and culture, Education services, Hotels, cafes and restaurants, and Insurance and other financial services. We then obtain the value of goods by subtracting the sum of these services categories. For both goods and services, we create nominal and real price

\footnotetext{
${ }^{10}$ See https://www.abs.gov.au/AUSSTATS/abs@.nsf/DetailsPage/5206.0Mar\%202020?0penDocument for an example of a March 2020 release of the national accounts.
} 
Figure A8: Goods and Services Inflation Stylized Facts - Australia

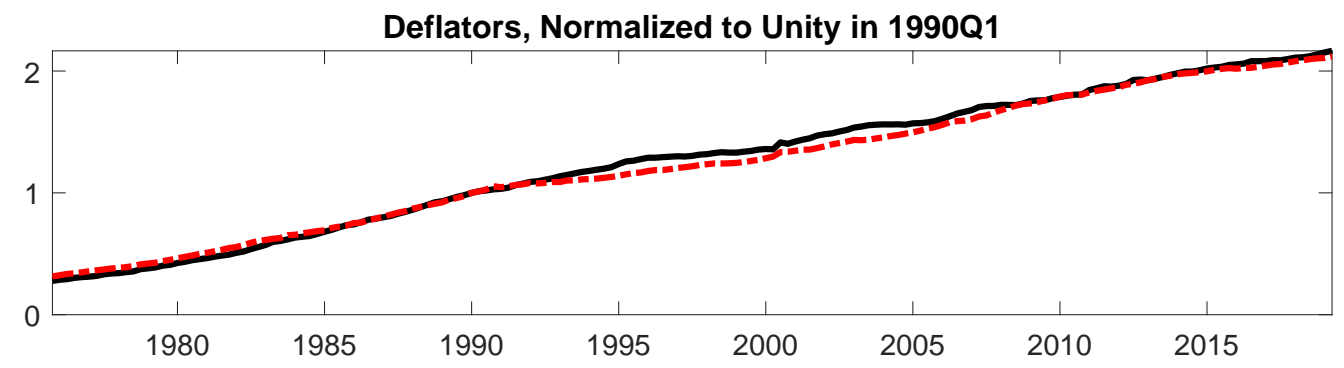

Year-on-Year Inflation Rate

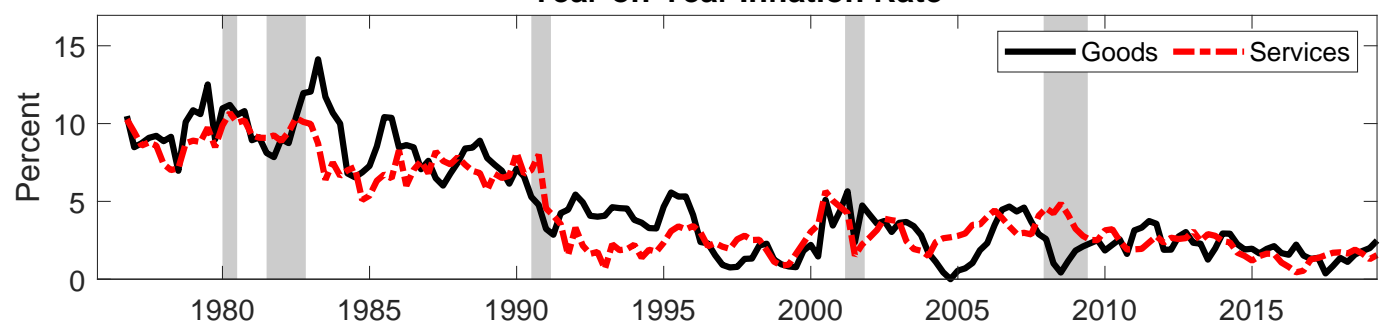

Share of Total Expenditure

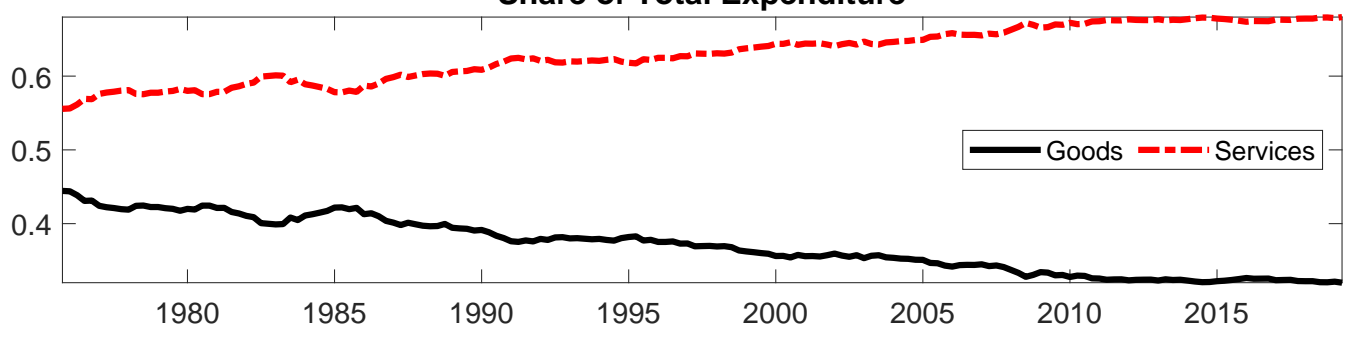

Notes: Expenditure shares sum to 100.

series by taking the appropriate categories from the ABS tables: the seasonally current prices (nominal) and seasonally adjusted chain-weighted volume measure (real). We then create the deflators by dividing the nominal measure by the real measure, then multiplying by 100. Sector-specific inflation rates are then obtained by once again taking the annualized difference of the natural logarithms.

Statistics Canada reports, as part of its quarterly national accounts, a breakdown of goods and services in its household final consumption expenditure. ${ }^{11}$ This breakdown means that the structure of the Canadian data exactly mimics the US data. We create sectorspecific deflators by dividing the measure of the current price by the measure of the real

\footnotetext{
${ }^{11}$ See http://www150.statcan.gc.ca/t1/tbl1/en/tv . action?pid=3610012401
} 
Figure A9: Goods and Services Inflation Stylized Facts - Canada

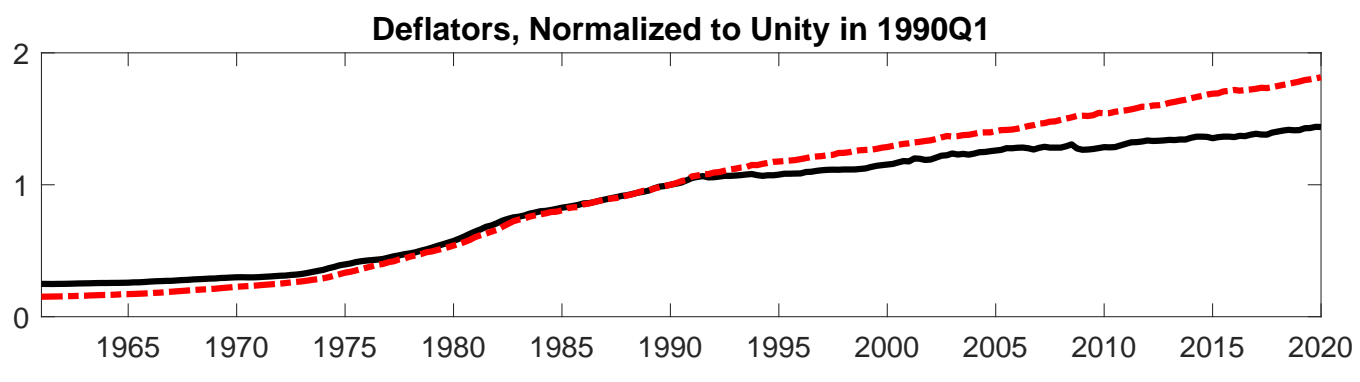

Year-on-Year Inflation Rate
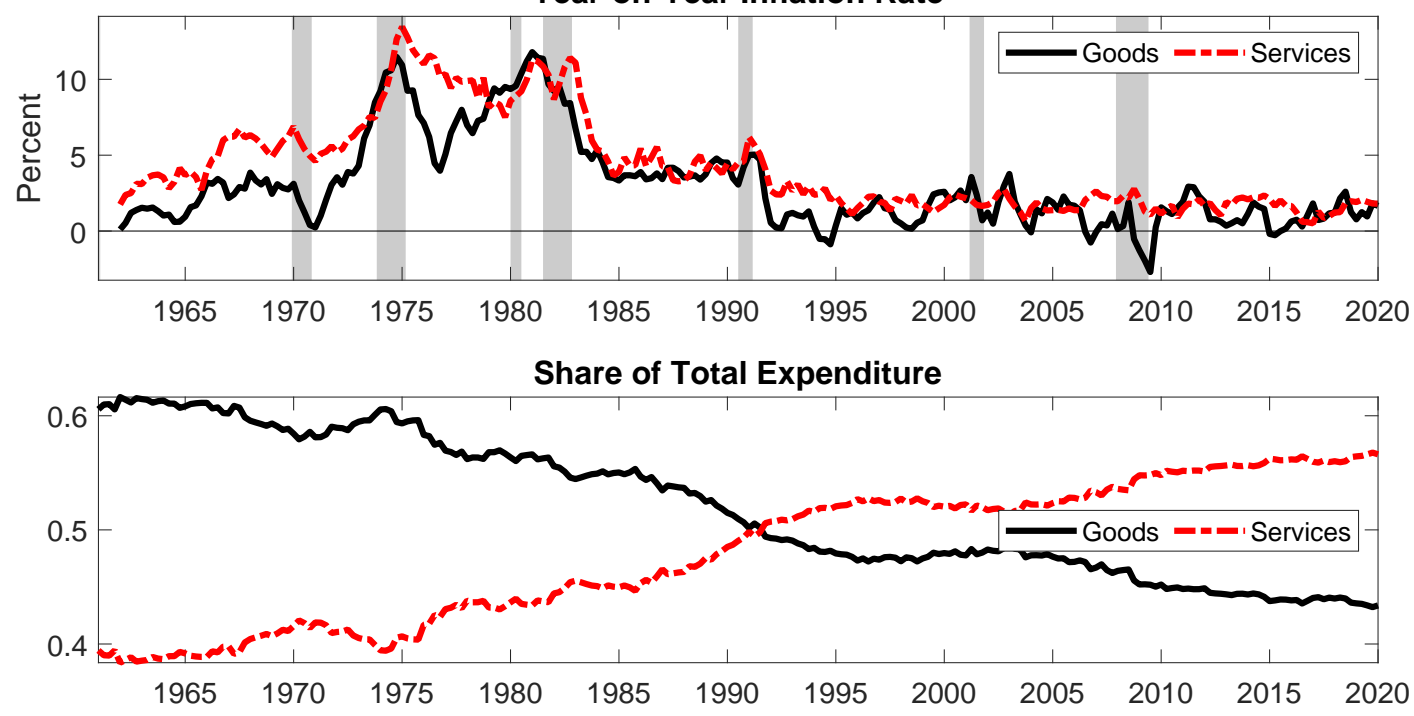

Notes: Expenditure shares sum to 100.

price. The sector-specific inflation rates are then constructed using the annualized difference of the natural logarithm of the deflator, which once again corresponds approximately to annualized quarter-on-quarter percentage point change in the sector-specific deflator.

Figures A8 and A9 plots for Australia and Canada, respectively, the constructed deflators (normalized to 1 for both sectors in 1990Q1), year-on-year inflation from the constructed deflators and the share of total consumption expenditure, mimicking Figure 1 in the main text. In general, the patterns for Canada look a lot like the US, in the sense that the goods deflator has flattened for the later part of the same. The pattern of goods and services inflation in Australia does look a bit different from US and Canada, in the sense that we do not see the goods deflator flattening substantially. For both Australia and Canada, we do 
see the services share rise through the sample, similar to what we observe for the US.

\section{A6.2 Other Australia and Canada Results}

Figure A10: Estimated Trend Inflation - Canada
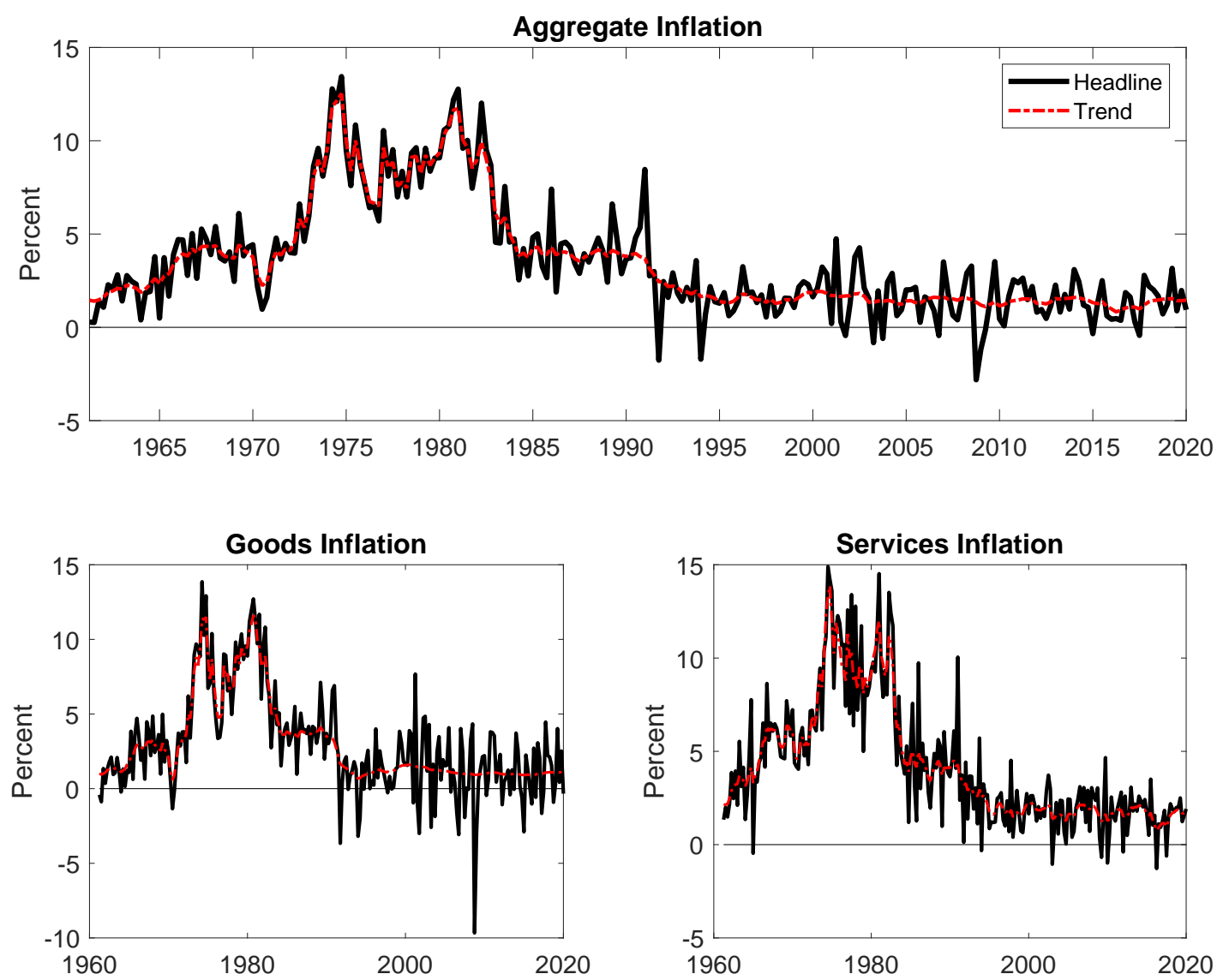

Notes: All units are in terms of annualized quarter-on-quarter inflation. Trend inflation estimates in terms of the posterior median estimate.

Figures A10 to A13 present various results which we obtain when we estimate our model on Australian and Canadian data. Figures A10 and A12 present the estimated posterior median estimate of the sector-specific and aggregate trend inflation together with actual sector-specific and aggregate inflation.

Figures A11 to A13 present the estimated standard deviation of innovations to trend goods and trend services, analogous to Figure 6 in the main text. In general, we observe 
Figure A11: Estimated Conditional Standard Deviation and Correlation of Innovations Canada
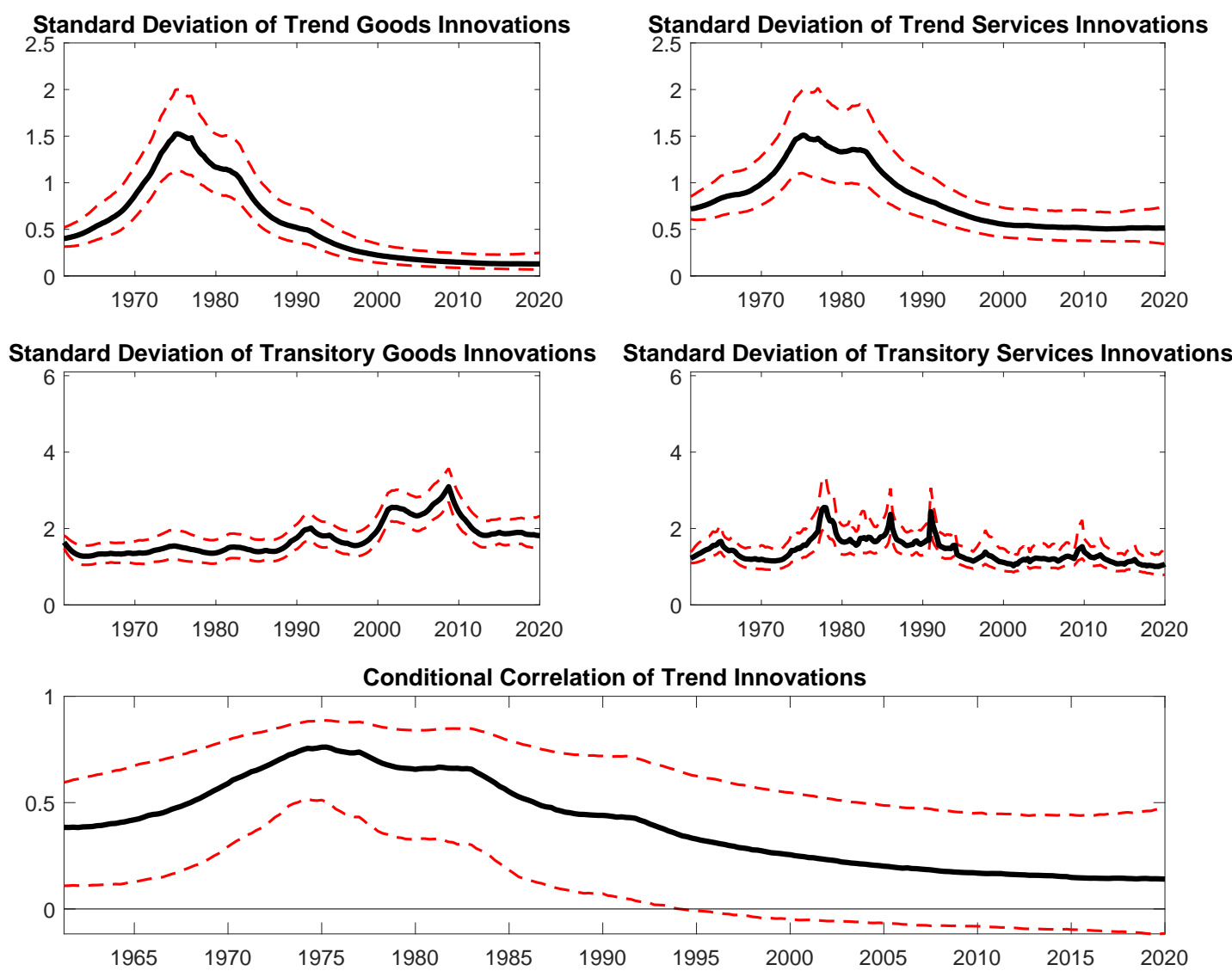

Notes: Posterior median estimate with associated $67 \%$ posterior credible interval.

the hump-shaped pattern in volatility as we see in the US. We also see the conditional correlation peak during the 1970s and 1980s for Canada and Australia, respectively, before this correlation disappeared in the 1990s. One difference is that the correlation is not very precisely estimated for Australia, with the $67 \%$ posterior credible set always containing zero.

As mentioned in the main text, the implication of all these results is that like the US, both Australia and Canada have seen the composition of variation in aggregate trend inflation change from being shared between goods and services to, since the 1990s, being almost fully dominated by variation in trend services inflation. 
Figure A12: Estimated Trend Inflation - Australia
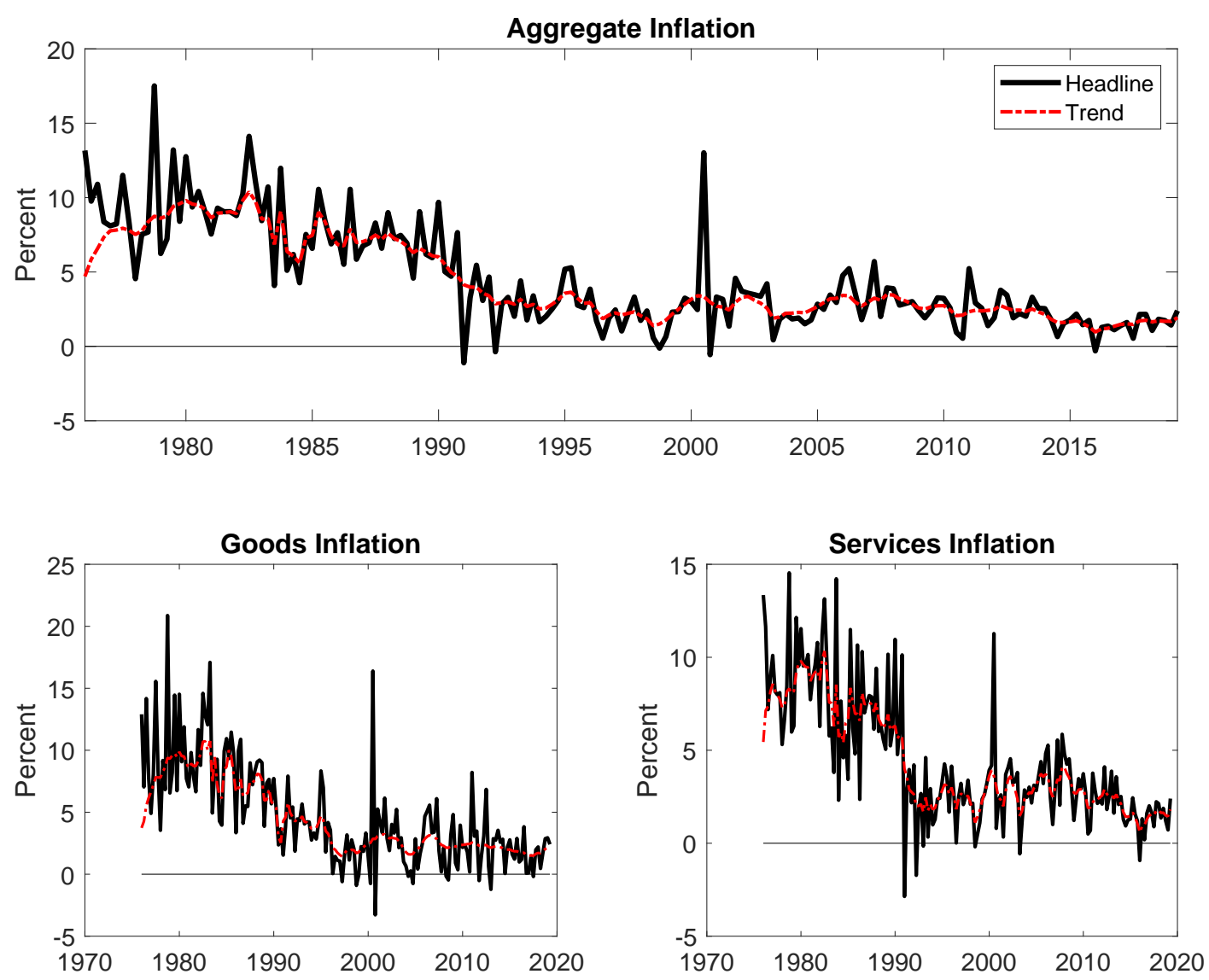

Notes: All units are in terms of annualized quarter-on-quarter inflation. Trend inflation estimates in terms of the posterior median estimate. 
Figure A13: Estimated Conditional Standard Deviation and Correlation of Innovations Australia
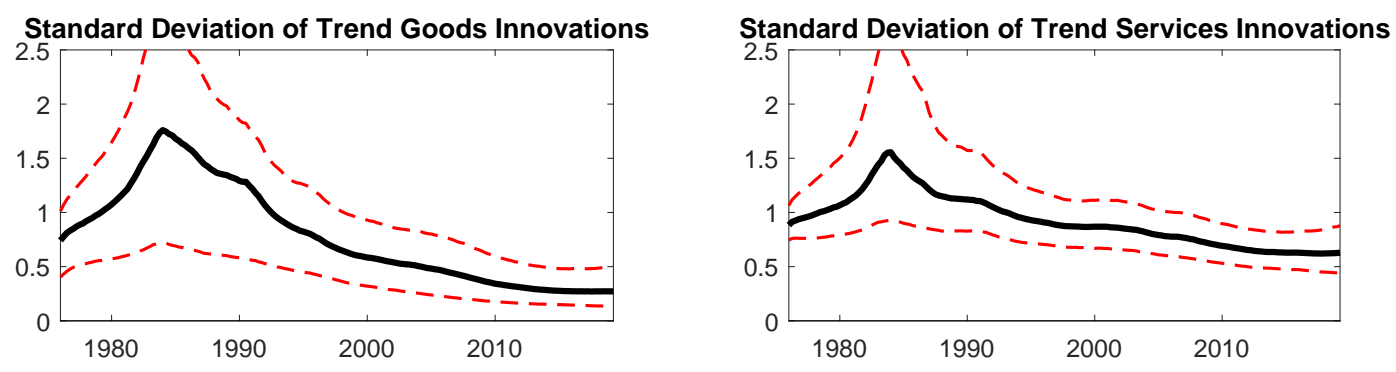

Standard Deviation of Transitory Goods Innovations

Standard Deviation of Transitory Services Innovations
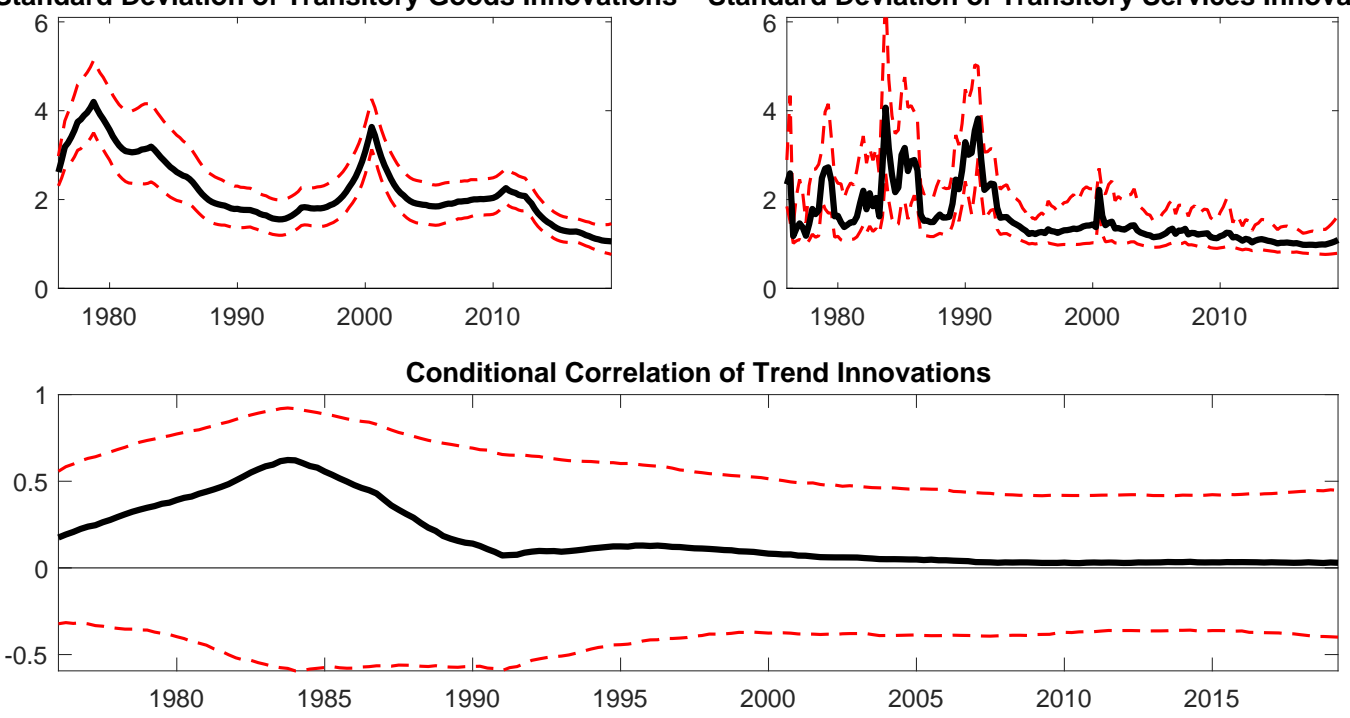

Notes: Posterior median estimate with associated $67 \%$ posterior credible interval. 


\section{References}

Anderson, Theodore W. 1984. An Introduction to Multivariate Statistical Analysis. Wiley.

Chan, Joshua C. C., Gary Koop, and Simon M. Potter. 2013. "A New Model of Trend Inflation." Journal of Business \& Economic Statistics, 31(1): 94-106.

Chan, Joshua C. C., Gary Koop, and Simon M. Potter. 2016. "A Bounded Model of Time Variation in Trend Inflation, Nairu and the Phillips Curve." Journal of Applied Econometrics, 31(3): 551-565.

Chan, Joshua CC, and Ivan Jeliazkov. 2009. "Efficient Simulation and Integrated Likelihood Estimation in State Space Models." International Journal of Mathematical Modelling and Numerical Optimisation, 1(1-2): 101-120.

Del Negro, Marco, and Giorgio E Primiceri. 2015. "Time Varying Structural Vector Autoregressions and Monetary Policy: A Corrigendum." The review of economic studies, 82(4): 1342-1345.

Diebold, Francis X, and Robert S Mariano. 1995. "Comparing predictive accuracy." Journal of Business $\& 3$ Economic Statistics, 13(3): 253-263.

Geweke, John, and Gianni Amisano. 2011. "Hierarchical Markov normal mixture models with applications to financial asset returns." Journal of Applied Econometrics, 26(1): 1-29.

Kim, Sangjoon, Neil Shephard, and Siddhartha Chib. 1998. "Stochastic Volatility: Likelihood Inference and Comparison with ARCH Models." The Review of Economic Studies, 65(3): 361-393.

Koop, Gary, Dale J Poirier, and Justin L Tobias. 2007. Bayesian Econometric Methods. Vol. 7, Cambridge University Press.

Kroese, Dirk P, Thomas Taimre, and Zdravko I Botev. 2013. Handbook of Monte Carlo Methods. Vol. 706, John Wiley \& Sons.

McCausland, William J, Shirley Miller, and Denis Pelletier. 2011. "Simulation Smoothing For State-Space Models: A Computational Efficiency Analysis." Computational Statistics \& Data Analysis, 55(1): 199-212.

Omori, Yasuhiro, Siddhartha Chib, Neil Shephard, and Jouchi Nakajima. 2007. "Stochastic Volatility with Leverage: Fast and Efficient Likelihood Inference." Journal of Econometrics, 140(2): 425-449. 
Raftery, Adrian E. 1995. "Bayesian Model Selection in Social Research." Sociological methodology, 111-163.

Reis, Ricardo, and Mark W. Watson. 2010. "Relative Goods' Prices, Pure Inflation, and the Phillips Correlation." American Economic Journal: Macroeconomics, 2(3): 128-157.

Stock, James H., and Mark W. Watson. 2007. "Why Has U.S. Inflation Become Harder to Forecast?" Journal of Money, Credit and Banking, 39(s1): 3-33.

Stock, James H., and Mark W. Watson. 2016. "Core Inflation and Trend Inflation." The Review of Economics and Statistics, 98(4): 770-784.

Whelan, Karl. 2000. "A guide to the use of chain aggregated NIPA data, Federal Reserve Board, Division of Research and Statistics." mimeo, June. 